\title{
Maximum Principles and Principal Eigenvalues
}

\author{
H. Amann \\ Institut für Mathematik, Universität Zürich, \\ Winterthurerstr. 190, CH-8057 Zürich, Switzerland
}

\begin{abstract}
In this paper well-known maximum principles are extended to second order cooperative linear elliptic systems with cooperative boundary conditions in strong, weak, and very weak settings. In addition, interrelations between maximum principles and principal eigenvalues are studied in detail, as well as continuity properties of principal eigenvalues under domain perturbations.
\end{abstract}

Key words: Maximum principles, principal eigenvalues, cooperative systems, cooperative boundary conditions, weak and very weak solutions, domain perturbations

\section{Introduction}

It is the main purpose of this paper to study maximum principles for linear second order cooperative elliptic systems under general linear first order cooperative boundary conditions. We are particularly interested in weak settings, in view of applications to nonlinear systems in situations where higher regularity either cannot be expected or does not constitute a convenient frame to deal with such problems.

Maximum principles for cooperative systems have already been discussed by several authors under various assumptions (cf. [20], [22], [32], [36], [43], [56], [60], [63], [71], [77]). However, in all these references, with the exception of [63], the case of Dirichlet boundary conditions is studied only. Furthermore, in almost all cases maximum principles in the strong sense are considered, that is, for $C^{2}$ functions, or, at least, for $W_{q}^{2}$ functions where $q$ is sufficiently large.

It is well-known that maximum principles are of great importance for the study of existence and qualitative properties of nonlinear equations. For example, one of the most 
useful techniques in the theory of second order scalar elliptic (and parabolic) boundary value problems, the method of sub- and supersolutions, is based on maximum principles (cf. [1], [62], [64], [67]). This is true for systems as well, as has already been observed in [1, Sections 5 and 10] and has since been worked out by several authors under various hypotheses (cf. [59], [62], [66], and the references therein). However, in all those papers either Dirichlet conditions are considered only or, if Neumann boundary conditions are studied at all, it is assumed that either the boundary conditions decouple, a rather particular situation (e.g., [40], [41]), or that very strong regularity conditions are satisfied (e.g., [62]). It is one of the advantages of our work that our maximum principles allow, among other things, comparison theorems for semilinear problems with nonlinear boundary conditions, the latter depending on all components of the unknown vector function, in a weak setting.

The validity of maximum principles is closely related to the existence of a principal eigenvalue, that is, of a least real eigenvalue determining the position of the smallest closed right half plane containing the spectrum. This eigenvalue plays a predominant rôle in the qualitative study of nonlinear boundary value problems via bifurcation theory and in the method of sub- and supersolutions (cf. [37], [51], [53], [54], [57], [58], and the references therein). Consequently, we investigate in some detail questions of existence and continuous dependence on the data of the principal eigenvalue.

It should be noted that our results on maximum principles in weak settings are new, even in the scalar case. The same is true for our continuity results for the principal eigenvalue, since we allow perturbations of the Robin boundary as well.

To give a flavor of the content of this paper we describe now some of our results in a simple setting. Here we restrict ourselves to a $2 \times 2$ system with the diagonal Laplace operator as principal part. The general case is studied in the main body of this work.

Throughout this paper $\Omega$ is a $C^{2}$ domain in $\mathbb{R}^{n}$, where $n \geq 1$, with a nonempty compact boundary $\Gamma$. We denote by $\nu:=\left(\nu^{1}, \ldots, \nu^{n}\right)$ the outer unit normal on $\Gamma$.

However, to illustrate some of the main results by means of prototypical examples, we assume throughout the rest of this introduction that $\Omega$ is bounded.

Let $u$ be a superharmonic distribution in $\Omega$, which means

$$
u \in \mathcal{D}^{\prime}(\Omega), \quad\langle-\Delta \varphi, u\rangle \geq 0 \quad \text { for all } \varphi \in \mathcal{D}(\Omega) \text { with } \varphi \geq 0 .
$$

Then it is known that $u$ is a regular distribution, in other words: $u \in L_{1, \text { loc }}(\Omega)$. If, moreover, for some point-wise defined representative $\widetilde{u}$ of $u$,

$$
\liminf _{\substack{x \in \Omega \\ x \rightarrow y}} \widetilde{u}(x) \geq 0 \quad \text { for all } y \in \Gamma,
$$

then $u \geq 0$, that is, $u(x) \geq 0$ for a.a. $x \in \Omega$ (e.g., [30, Propositions II.4.20 and II.4.21]). It is clear that from (1) alone nothing can be said about the boundary behavior of $u \in$ $L_{1, \text { loc }}(\Omega)$ since every test function $\varphi \in \mathcal{D}(\Omega)$ vanishes near $\Gamma$. Thus (1), without the additional information of (2), does not imply that $u \geq 0$. 
The situation is different if we require the validity of the inequalities in (1) for a larger class of test functions and a little more regularity for $u$. For this, given $q \in(1, \infty)$, we put

$$
W_{q^{\prime}, \gamma}^{2}(\Omega, \mathbb{R}):=\left\{u \in W_{q^{\prime}}^{2}(\Omega, \mathbb{R}) ; \gamma u=0\right\},
$$

where

$$
\frac{1}{q}+\frac{1}{q^{\prime}}=1
$$

and $\gamma$ is the trace operator. We also denote by $\langle\cdot, \cdot\rangle$ the usual $L_{q}$ duality pairing. Then it is a consequence of our much more general results that the following very weak maximum principle (rather: minimum principle) is valid:

$$
\left.\begin{array}{l}
u \in L_{q}(\Omega, \mathbb{R}) \text { for some } q \in(1, \infty), \\
\langle-\Delta v, u\rangle \geq 0 \text { for all } v \in W_{q^{\prime}, \gamma}^{2}(\Omega, \mathbb{R}) \text { with } v \geq 0
\end{array}\right\} \quad \text { imply } u \geq 0 \text {. }
$$

Very weak maximum principles are of importance in nonlinear problems involving low regularity data, for example (e.g., [12]). The maximum principles studied below are valid for cooperative systems also. To illustrate this we consider the model system $(\mathcal{A}, \mathcal{B})$ on $\Omega$ defined as follows: we put $u:=\left(u^{1}, u^{2}\right)$ and assume that there are two decompositions of $\Gamma$ :

$$
\Gamma=\Gamma_{0}^{1} \cup \Gamma_{1}^{1}=\Gamma_{0}^{2} \cup \Gamma_{1}^{2} \quad \Gamma_{0}^{1} \cap \Gamma_{1}^{1}=\Gamma_{0}^{2} \cap \Gamma_{1}^{2}=\varnothing,
$$

such that $\Gamma_{0}^{1}$ and $\Gamma_{0}^{2}$ are open, hence closed, submanifolds of $\Gamma$. Then we define

$$
\mathcal{A} u=\left(\mathcal{A}^{1} u, \mathcal{A}^{2} u\right)
$$

by

$$
\begin{aligned}
\mathcal{A}^{1} u & :=-\Delta u^{1}+a^{11} u^{1}+a^{12} u^{2}, \\
\mathcal{A}^{2} u & :=-\Delta u^{2}+a^{21} u^{1}+a^{22} u^{2},
\end{aligned}
$$

and

$$
\mathcal{B} u=\left(\mathcal{B}^{1} u, \mathcal{B}^{2} u\right)
$$

by

$$
\mathcal{B}^{1} u:= \begin{cases}u^{1} & \text { on } \Gamma_{0}^{1} \\ \partial_{\nu} u^{1}+b^{11} u^{1}+b^{12} u^{2} & \text { on } \Gamma_{1}^{1}\end{cases}
$$

and

$$
\mathcal{B}^{2} u:= \begin{cases}u^{2} & \text { on } \Gamma_{0}^{2} \\ \partial_{\nu} u^{2}+b^{21} u^{1}+b^{22} u^{2} & \text { on } \Gamma_{1}^{2}\end{cases}
$$

where we assume that

$$
a^{r s} \in L_{\infty}(\Omega, \mathbb{R}), \quad b^{r s} \in C^{1-}(\Gamma, \mathbb{R}), \quad r, s \in\{1,2\},
$$

with the hypothesis of 'cooperativity':

$$
a^{12} \leq 0, \quad a^{21} \leq 0, \quad b^{12} \leq 0, \quad b^{21} \leq 0 .
$$

As usual, $C^{1-}$ means 'Lipschitz continuous'. 
Denoting by $\chi^{r}$ the characteristic function of $\Gamma_{1}^{r}$ and introducing matrix notation,

$$
\chi:=\left[\begin{array}{cc}
\chi^{1} & 0 \\
0 & \chi^{2}
\end{array}\right], \quad a:=\left[\begin{array}{cc}
a^{11} & a^{12} \\
a^{21} & a^{22}
\end{array}\right], \quad b:=\left[\begin{array}{ll}
b^{11} & b^{12} \\
b^{21} & b^{22}
\end{array}\right],
$$

we can rewrite this system in the concise from

$$
\begin{aligned}
& \mathcal{A} u=-\Delta u+a u, \\
& \mathcal{B} u=\chi\left(\partial_{\nu} u+b u\right)+(1-\chi) u
\end{aligned}
$$

Of course, the boundary operator is to be understood in the sense of traces.

We also define the formally adjoint problem $\left(\mathcal{A}^{\sharp}, \mathcal{B}^{\sharp}\right)$ by

$$
\begin{aligned}
& \mathcal{A}^{\sharp} v:=-\Delta v+a^{\top} v, \\
& \mathcal{B}^{\sharp} v:=\chi\left(\partial_{\nu} v+b^{\top} v\right)+(1-\chi) v,
\end{aligned}
$$

where $a^{\top}$ is the transposed of $a$, etc.

We endow all spaces of functions with their natural point- and component-wise defined order.

First we consider the eigenvalue problem

$$
\mathcal{A} u=\lambda u \text { in } \Omega, \quad \mathcal{B} u=0 \text { on } \Gamma .
$$

It will be shown that every eigenfunction of $(\mathcal{A}, \mathcal{B})$, that is, of $(5)$, associated with any eigenvalue $\lambda$ is regular in the sense that it belongs to

$$
W_{\infty-}^{2}\left(\Omega, \mathbb{C}^{2}\right):=\bigcap_{1<q<\infty} W_{q}^{2}\left(\Omega, \mathbb{C}^{2}\right) .
$$

Furthermore, our results guarantee that $(\mathcal{A}, \mathcal{B})$, that is, problem $(5)$, possesses a least real eigenvalue, the principal eigenvalue, $\lambda_{0}(\mathcal{A}, \mathcal{B})$, of $(\mathcal{A}, \mathcal{B})$, and it is associated with a positive eigenfunction.

Although there may exist other real eigenvalues of $(\mathcal{A}, \mathcal{B})$ possessing positive eigenfunctions, the principal eigenvalue characterizes the validity of the maximum principle for $(\mathcal{A}, \mathcal{B})$. More precisely, for $1<q<\infty$ we put

$$
W_{q^{\prime}, \mathcal{B}}^{2}\left(\Omega, \mathbb{R}^{2}\right):=\left\{v \in W_{q^{\prime}}^{2}\left(\Omega, \mathbb{R}^{2}\right) ; \mathcal{B}^{\sharp} v=0\right\} .
$$

We say that $(\mathcal{A}, \mathcal{B})$ satisfies the very weak maximum principle if

$$
\left.\begin{array}{l}
u \in L_{q}\left(\Omega, \mathbb{R}^{2}\right) \text { for some } q \in(1, \infty), \\
\left\langle\mathcal{A}^{\sharp} v, u\right\rangle \geq 0 \text { for all } v \in W_{q^{\prime}, \mathcal{B}}^{2}\left(\Omega, \mathbb{R}^{2}\right) \text { with } v \geq 0
\end{array}\right\} \quad \text { imply } u \geq 0 .
$$

Theorem 1. $(\mathcal{A}, \mathcal{B})$ satisfies the very weak maximum principle iff $\lambda_{0}(\mathcal{A}, \mathcal{B})>0$. 
Since the principal eigenvalue of the Dirichlet Laplacean is positive, Theorem 1 is an extension of (3) to system (4).

As already mentioned, this theorem is new, even in the well-studied scalar case (where obvious analogues of the theorems of this introduction are valid). Indeed, to the best of our knowledge the very weak maximum principle has not been observed so far. Also note that there is no restriction on $q$, besides $1<q<\infty$.

In order to guarantee that the principle eigenvalue is the only one with a positive eigenfunction we have to impose an additional condition. For this, the pair $(a, b)$, more precisely: $(a, \chi b \chi)$, is said to be irreducible if

$$
b^{12} \mid \Gamma_{1}^{1} \cap \Gamma_{1}^{2}=0 \text { implies } a^{12} \neq 0
$$

and

$$
b^{21} \mid \Gamma_{1}^{1} \cap \Gamma_{1}^{2}=0 \text { implies } a^{21} \neq 0,
$$

putting

$$
b^{12}\left|\varnothing:=b^{21}\right| \varnothing:=0
$$

Note that these conditions can be rewritten as

$$
\begin{array}{lll}
a^{12} \neq 0 & \text { if } & \chi^{1} b^{12} \chi^{2}=0 \\
a^{21} \neq 0 & \text { if } & \chi^{2} b^{21} \chi^{1}=0 .
\end{array}
$$

For example, the pair $(a, b)$ with

$$
a=\left[\begin{array}{ll}
a^{11} & 0 \\
a^{21} & a^{22}
\end{array}\right], \quad b=\left[\begin{array}{ll}
b^{11} & b^{12} \\
0 & b^{22}
\end{array}\right]
$$

is irreducible if $a^{21} \neq 0$ and $b^{12} \mid \Gamma_{1}^{1} \cap \Gamma_{1}^{2} \neq 0$. Then the following improvement over the mere existence of a principal eigenvalue with a positive eigenfunction is valid.

Theorem 2. Let $(a, b)$ be irreducible. Then $\lambda_{0}(\mathcal{A}, \mathcal{B})$ is a simple eigenvalue of $(\mathcal{A}, \mathcal{B})$ and the only one with a positive eigenfunction.

We refer to the main body of this paper for a precise definition of the simplicity of an eigenvalue of $(\mathcal{A}, \mathcal{B})$ and for further properties of $\lambda_{0}(\mathcal{A}, \mathcal{B})$ and the associated eigenfunction.

If $(a, b)$ is irreducible then we obtain another useful characterization of the positivity of the principal eigenvalue. For this we say that $u$ is a very weak strict supersolution for $(\mathcal{A}, \mathcal{B})$, provided

$$
\begin{aligned}
& u \in L_{q}\left(\Omega, \mathbb{R}^{2}\right) \text { for some } q \in(1, \infty) \text { and } \\
& \left\langle\mathcal{A}^{\sharp} v, u\right\rangle \geq 0 \text { for all } v \in W_{q^{\prime}, \mathcal{B}^{\sharp}}^{2} \text { with } v \geq 0, \\
& \text { with a strict inequality sign for at least one } v .
\end{aligned}
$$


It follows from Green's formula that $u$ is a very weak strict supersolution for $(\mathcal{A}, \mathcal{B})$ if $u \in W_{q}^{2}\left(\Omega, \mathbb{R}^{2}\right)$ for some $q \in(1, \infty)$ and $(\mathcal{A} u, \mathcal{B} v)>0$, meaning, of course, that $\mathcal{A} u \geq 0$ in $\Omega, \mathcal{B} u \geq 0$ on $\Gamma$, and $(\mathcal{A} u, \mathcal{B} u) \neq(0,0)$.

Theorem 3. Let $(a, b)$ be irreducible. Then $(\mathcal{A}, \mathcal{B})$ satisfies the very weak maximum principle iff there exists a positive very weak strict supersolution for $(\mathcal{A}, \mathcal{B})$.

Theorems 2 and 3 (and their more general versions presented in Sections 6 and 7) generalize considerably the results of [56] and [71]. Indeed, besides of the fact that those authors consider only Dirichlet boundary conditions, our regularity hypotheses are substantially weaker than theirs. In particular, in Theorem 3 we are dealing with very weak supersolutions only.

In the following section we formulate the hypotheses used throughout most of this paper and give a precise formulation of the differential operators under consideration. In Section 3 we fix some general notations and describe the boundary spaces for our systems.

Our main results - very weak, weak, and strong maximum principles and their interrelations as well as monotonicity and continuity properties of the principal eigenvalue are contained in Sections 4-11, where only the more elementary proofs are given. The somewhat deeper statements as well as additional results are proved in Sections 15-18.

In Section 12 we collect some functional analytical tools, and in Section 13 we recall the version of the maximum principle for scalar equations from which we derive our results for systems. Section 14 contains the fundamental solvability results for nonhomogeneous problems in the strong, weak, and very weak setting.

For all these results we impose enough regularity on the coefficients of the differential operators to guarantee that the assertions are independent of $q \in(1, \infty)$. In Section 19 we present weak maximum principles in $W_{q}^{1}$, assuming minimal $q$-dependent regularity only. They lead to comparison theorems for semilinear elliptic boundary value problems which are of importance in the study of such problems in situations where strong solutions do not exist.

We also show that the various realizations of cooperative elliptic systems generate positive analytic semigroups. These results have important implications for parabolic problems. Since this paper is already rather long we refrain from giving details.

\section{Elliptic boundary value problems}

In this section we give precise formulations of the elliptic problems under consideration and state the hypotheses used throughout the following, unless explicitly stated otherwise.

We assume that

$$
N \in \mathbb{N}^{\times}:=\mathbb{N} \backslash\{0\}
$$


The space of real $N \times N$ matrices, $a=\left[a^{r s}\right]$, is denoted by $\mathbb{R}^{N \times N}$, and $\mathbb{R}_{\mathrm{diag}}^{N \times N}$ is the linear subspace of all diagonal matrices,

$$
a=\operatorname{diag}\left[a^{1}, \ldots, a^{N}\right] .
$$

We always use the summation convention with respect to $j$ and $k$ belonging to $\{1, \ldots, n\}$.

We also assume that

- $a_{j k}=a_{k j} \in B U C^{1}\left(\Omega, \mathbb{R}_{\mathrm{diag}}^{N \times N}\right), 1 \leq j, k \leq n$;

- $\boldsymbol{a}^{r}(x):=\left[a_{j k}^{r}(x)\right] \in \mathbb{R}^{n \times n}$ is positive definite for $1 \leq r \leq N$, uniformly with respect to $x \in \Omega$;

- $a_{j} \in W_{\infty}^{1}\left(\Omega, \mathbb{R}_{\mathrm{diag}}^{N \times N}\right), 1 \leq j \leq n$;

- $a \in L_{\infty}\left(\Omega, \mathbb{R}^{N \times N}\right), b \in C^{1-}\left(\Gamma, \mathbb{R}^{N \times N}\right)$;

- $-a$ and $-b$ are cooperative, that is, $a^{r s} \leq 0$ and $b^{r s} \leq 0$ for $r \neq s$.

Observe that there are no sign restrictions for the diagonal entries, neither for $a$ nor for $b$.

We consider the elliptic differential operator $\mathcal{A}$ acting on $\mathbb{R}^{N}$-valued distributions

$$
u:=\left(u^{1}, \ldots, u^{N}\right)
$$

on $\Omega$, defined by

$$
\mathcal{A} u:=-\partial_{j}\left(a_{j k} \partial_{k} u\right)+a_{j} \partial_{j} u+a u \text {. }
$$

Thus

where

$$
\mathcal{A} u=\left(\mathcal{A}^{1} u, \ldots, \mathcal{A}^{N} u\right),
$$

$$
\mathcal{A}^{r} u=-\partial_{j}\left(a_{j k}^{r} \partial_{k} u^{r}\right)+a_{j}^{r} \partial_{j} u^{r}+\sum_{s=1}^{N} a^{r s} u^{s} .
$$

Note that $\mathcal{A}$ has diagonal principal and first order parts, but is coupled in its lowest order terms.

We fix

$$
\chi \in C\left(\Gamma, \mathbb{R}_{\mathrm{diag}}^{N \times N}\right) \quad \text { with } \chi^{r}(y) \in\{0,1\} \text { for } y \in \Gamma \text { and } 1 \leq r \leq N,
$$

a boundary identification map for $\Omega$. Hence

$$
\Gamma_{i}^{r}:=\left(\chi^{r}\right)^{-1}(i), \quad i \in\{0,1\},
$$

are open in $\Gamma$ and disjoint with union $\Gamma$ for $1 \leq r \leq N$. Then we define a boundary operator $\mathcal{B}$ by

$$
\mathcal{B} u:=\chi\left(\partial_{\mu} u+b u\right)+(1-\chi) u,
$$

where

$$
\partial_{\mu}:=\operatorname{diag}\left[\partial_{\mu^{1}}, \ldots, \partial_{\mu^{N}}\right]
$$

with $\mu^{r}:=\boldsymbol{a}^{r} \nu$ being the outer conormal with respect to $\boldsymbol{a}^{r}$. Of course, $\mathcal{B}$ is to be understood in the sense of traces. It follows that

$$
\mathcal{B} u=\left(\mathcal{B}^{1} u, \ldots, \mathcal{B}^{N} u\right)
$$


where

$$
\mathcal{B}^{r} u= \begin{cases}u^{r} & \text { on } \Gamma_{0}^{r}, \\ \partial_{\mu^{r}} u^{r}+\sum_{s=1}^{N} b^{r s} u^{s} & \text { on } \Gamma_{1}^{r}\end{cases}
$$

Thus $\mathcal{B}^{r} u$ is the Dirichlet boundary operator on $\Gamma_{0}^{r}$ for the $r$-th component $u^{r}$ of $u$, and a Neumann or Robin boundary operator for $u^{r}$ on $\Gamma_{1}^{r}$. Note, however, that these boundary operators are coupled in their lowest order terms unless

$$
\Gamma_{0}^{1}=\cdots=\Gamma_{0}^{N}=\Gamma,
$$

that is, unless $\mathcal{B}=\gamma$, the Dirichlet boundary operator. We express these facts by saying that $(\mathcal{A}, \mathcal{B})$ is a (weakly coupled second order) cooperative elliptic boundary value problem $($ on $\Omega$ ).

If $n=1$ then either $\Gamma=\left\{x_{0}\right\}$ or $\Gamma=\left\{x_{0}, x_{1}\right\}$ with $x_{i} \in \mathbb{R}$. Thus every space of $\mathbb{R}^{N}$ valued functions on $\Gamma$ is naturally isomorphic to either $\mathbb{R}^{N}$ or $\mathbb{R}^{N} \times \mathbb{R}^{N}$, and all considerations of this paper apply with the obvious interpretations.

We put

$$
\mathcal{A}^{\sharp} v:=-\partial_{j}\left(a_{j k} \partial_{k} v+a_{j} v\right)+a^{\top} v
$$

and

$$
\mathcal{B}^{\sharp} v:=\chi\left[\partial_{\mu} v+\left(\nu^{j} a_{j}+b^{\top}\right) v\right]+(1-\chi) v .
$$

Then $\left(\mathcal{A}^{\sharp}, \mathcal{B}^{\sharp}\right)$ is the elliptic boundary value problem formally adjoint to $(\mathcal{A}, \mathcal{B})$. We also put

$$
\langle u, v\rangle:=\int_{\Omega} u \cdot v d x, \quad(u, v) \in L_{q^{\prime}}\left(\Omega, \mathbb{R}^{N}\right) \times L_{q}\left(\Omega, \mathbb{R}^{N}\right) .
$$

Similarly, $\langle\cdot, \cdot\rangle_{\Gamma}$ is the $L_{q}(\Gamma)$ duality pairing, obtained by replacing $\Omega$ and $d x$ in (10) by $\Gamma$ and $d \sigma$, respectively, $d \sigma$ being the volume measure of $\Gamma$. Then the Dirichlet form $\mathfrak{a}$ of $(\mathcal{A}, \mathcal{B})$ is defined by

$$
\mathfrak{a}(v, u):=\left\langle\partial_{j} v, a_{j k} \partial_{k} u\right\rangle+\left\langle v, a_{j} \partial_{j} u+a u\right\rangle+\langle\gamma v, \chi b \gamma u\rangle_{\Gamma}
$$

for $(v, u) \in W_{q^{\prime}}^{1}\left(\Omega, \mathbb{R}^{N}\right) \times W_{q}^{1}\left(\Omega, \mathbb{R}^{N}\right)$.

\section{Notations and conventions}

Henceforth, as long as $\Omega$ is kept fixed, we use the following simplified notation: if $E\left(\Omega, \mathbb{R}^{N}\right)$ is a vector subspace of

$$
L_{1, \text { loc }}:=L_{1, \text { loc }}\left(\Omega, \mathbb{R}^{N}\right)
$$

then we denote it simply by $E$. For example,

$$
W_{q}^{k}:=W_{q}^{k}\left(\Omega, \mathbb{R}^{N}\right) \text { for } k \in \mathbb{N} \text {. }
$$

Similarly, we simply write $E(\Gamma)$ for $E\left(\Gamma, \mathbb{R}^{N}\right)$ if the latter is a vector subspace of

$$
L_{1}(\Gamma):=L_{1}\left(\Gamma, \mathbb{R}^{N}\right) .
$$


We endow $L_{1, \text { loc }}(\Omega, \mathbb{R})$ with its natural 'point-wise' order induced by the positive cone

$$
L_{1, \text { loc }}^{+}(\Omega, \mathbb{R}):=\left\{u \in L_{1, \text { loc }}(\Omega, \mathbb{R}) ; u(x) \geq 0 \text { a.a. } x \in \Omega\right\} .
$$

Similarly, $L_{1}(\Gamma, \mathbb{R})$ is ordered by $L_{1}^{+}(\Gamma, \mathbb{R})$, the latter being defined in analogy to (11), but with respect to the volume measure $d \sigma$ of $\Gamma$.

If $E$ is an ordered vector space then we write $E^{+}$for its positive (proper) cone and $e>0$ means $e \geq 0$ but $e \neq 0$, of course. If $F$ is a linear subspace of $E$ then $F$ is given the induced order whose positive cone is $F^{+}:=E^{+} \cap F$. Furthermore, if $E_{1}, \ldots, E_{m}$ are ordered vector spaces then $E_{1} \times \cdots \times E_{m}$ is given the product order, that is, $\left(e_{1}, \ldots, e_{m}\right) \geq 0$ iff $e_{i} \geq 0$ for $1 \leq i \leq m$. Consequently, every vector subspace $E$ of $L_{1, \text { loc }}$, or of $L_{1}(\Gamma)$, is an ordered vector space with respect to the naturally induced 'point-wise' product order. If $E$ and $F$ are ordered vector spaces then a linear map $T$ from $E$ into $F$ is said to be positive, we write $T>0$, if $T\left(E^{+}\right) \subset F^{+}$. Note, for example, that, consequently, $a \geq 0$ for $a \in L_{\infty}\left(\Omega, \mathbb{R}^{N \times N}\right)$ means that $a^{r s}(x) \geq 0$ for $1 \leq r, s \leq N$ and a.a. $x \in \Omega$.

As a rule, in this paper all vector spaces are over the reals. However, if there occur, explicitly or implicitly, complex numbers in a given formula then it is always understood that the corresponding statement refers to the complexified version of that formula. For example, if $A$ is a linear operator in a (real) Banach space then $\sigma(A), \sigma_{p}(A)$, and $\rho(A)$ denote the spectrum, the point spectrum, and the resolvent set, respectively, of the complexification of $A$. For $\lambda \in \sigma_{p}(A)$ we denote by $N_{A}(\lambda)$ the algebraic eigenspace,

$$
N_{A}(\lambda):=\bigcup_{k=1}^{\infty} \operatorname{ker}\left[(\lambda-A)^{k}\right],
$$

of $\lambda$. Recall that $\operatorname{dim}\left(N_{A}(\lambda)\right)$ is the (algebraic) multiplicity of $A$, and $\lambda$ is a simple eigenvalue if its multiplicity equals 1 .

Let $E$ and $F$ be Banach spaces. Then $\mathcal{L}(E, F)$ is the Banach space of all bounded linear maps from $E$ into $F$, and $\mathcal{L}(E):=\mathcal{L}(E, E)$. Moreover, $\mathcal{L}$ is $(E, F)$ is the set of all isomorphisms in $\mathcal{L}(E, F)$. If

$$
E \stackrel{d}{\hookrightarrow} F
$$

that is, $E$ is continuously and densely embedded in $F$, then $\mathcal{H}(E, F)$ is the subset of all $A \in \mathcal{L}(E, F)$ such that $-A$, considered as a linear operator in $F$ with domain $E$, generates a strongly continuous analytic semigroup, denoted by

$$
\mathcal{U}_{A}:=\left\{U_{A}(t) ; t \geq 0\right\},
$$

on $F$, that is, in $\mathcal{L}(F)$. Given $A \in \mathcal{H}(E, F)$, there exists $\omega \in \mathbb{R}$ such that $[\operatorname{Re} z>\omega]$ belongs to $\rho(-A)$, and the infimum of all such $\omega$ is the spectral bound, $s(-A)$, of $A$ (e.g., $[8$, Section I.1.2]). We also set

$$
\lambda_{0}(A):=-s(-A)=\inf \{\operatorname{Re} \lambda ; \lambda \in \sigma(A)\},
$$

where $\inf (\varnothing):=\infty$. If $E$ is an ordered Banach space $(O B S)$ then the semigroup $\mathcal{U}_{A}$ is said to be positive if $U_{A}(t) \geq 0$ for $t \geq 0$. 
We always assume that

$$
1<q<\infty
$$

For $|s| \leq 2$ we put

$$
W_{q}^{s}\left(\Gamma_{j}\right):=\prod_{r=1}^{N} W_{q}^{s}\left(\Gamma_{j}^{r}\right), \quad j=0,1,
$$

with the understanding that $W_{q}^{s}(\varnothing):=\{0\}$. Then $W_{q}^{s}\left(\Gamma_{j}\right)$ is an ordered Banach space with its point-wise order if $s \geq 0$. If $s<0$ then we endow $W_{q}^{s}\left(\Gamma_{j}\right)$ with the natural dual order whose positive cone is the dual (that is, polar) of $W_{q^{\prime}}^{-s}\left(\Gamma_{j}\right)^{+}$. Then the injection maps

$$
W_{q}^{s}\left(\Gamma_{j}\right) \stackrel{d}{\hookrightarrow} W_{q}^{t}\left(\Gamma_{j}\right), \quad-2 \leq t<s \leq 2, \quad j=0,1,
$$

are well-defined and positive. Finally, we put

$$
\partial W_{q}^{s}:=W_{q}^{s-1 / q}\left(\Gamma_{0}\right) \oplus W_{q}^{s-1-1 / q}\left(\Gamma_{1}\right), \quad 0 \leq s \leq 2 .
$$

This means that we consider $W_{q}^{s-1}\left(\Gamma_{0}\right)$ and $W_{q}^{s-1-1 / q}\left(\Gamma_{1}\right)$ as linear subspaces of $W_{q}^{s-1-1 / q}(\Gamma)$, by extending the corresponding elements by zero over $\Gamma$, and endow their algebraic direct sum

$$
\Sigma:=W_{q}^{s-1 / q}\left(\Gamma_{0}\right)+W_{q}^{s-1-1 / q}\left(\Gamma_{1}\right)
$$

with the unique topology for which

$$
W_{q}^{s-1 / q}\left(\Gamma_{0}\right) \times W_{q}^{s-1-1 / q}\left(\Gamma_{1}\right) \rightarrow \Sigma, \quad\left(g_{0}, g_{1}\right) \mapsto g_{0}+g_{1}
$$

is a topological isomorphism. Then $\partial W_{q}^{s}$ is an ordered Banach space with the unique order for which (12) and its inverse are positive.

\section{Weak maximum principles}

Using Sobolev embeddings, the trace theorem, and Hölder's inequality, it follows that

$$
(\mathcal{A}, \mathcal{B}) \in \mathcal{L}\left(W_{q}^{2}, L_{q} \times \partial W_{q}^{2}\right)
$$

Thus

$$
W_{q, \mathcal{B}}^{2}:=\left\{u \in W_{q}^{2} ; \mathcal{B} u=0\right\}
$$

is a closed linear subspace of $W_{q}^{2}$, hence a Banach space with

$$
W_{q, \mathcal{B}}^{2} \stackrel{d}{\hookrightarrow} L_{q}
$$

We denote by $A:=A_{(q)}$ the $L_{q}$ realization of $(\mathcal{A}, \mathcal{B})$, defined by $A:=\mathcal{A} \mid W_{q, \mathcal{B}}^{2}$. Similarly, $A^{\sharp}$ is the $L_{q^{\prime}}$ realization of $\left(\mathcal{A}^{\sharp}, \mathcal{B}^{\sharp}\right)$.

Theorem 4. $A \in \mathcal{H}\left(W_{q, \mathcal{B}}^{2}, L_{q}\right)$ and $A^{\sharp} \in \mathcal{H}\left(W_{q^{\prime}, \mathcal{B}^{\sharp}}^{2}, L_{q^{\prime}}\right)$ for $1<q<\infty$. Moreover, $A^{\sharp}=A^{\prime}$. 
Proof. The first assertion is well-known (cf. [7, Theorem 4.1] and the references therein; also see [34]). Since $\gamma a_{j} \in C^{\rho}\left(\Gamma, \mathbb{R}^{N \times N}\right)$ for every $\rho \in(0,1)$, it follows from the preceding references that

$$
A^{\sharp} \in \mathcal{H}\left(W_{q^{\prime}, \mathcal{B}^{\sharp}}^{2}, L_{q^{\prime}}\right) \text {. }
$$

The last assertion is a consequence of Green's formula and the fact that $A$ and $A \sharp$ have a common point in their resolvent sets (even a half-plane, of course).

\section{Remarks 5.}

(a) The assumption that $a_{j} \in W_{\infty}^{1}\left(\Omega, \mathbb{R}^{N \times N}\right)$ guarantees that the assertions about $A^{\sharp}$ are valid. If we are only interested in

$$
A \in \mathcal{H}\left(W_{q, \mathcal{B}}^{2}, L_{q}\right)
$$

and not in the explicit representation of $A^{\prime}$ then it suffices to suppose that

$$
a_{j} \in L_{\infty}\left(\Omega, \mathbb{R}_{\text {diag }}^{N \times N}\right), \quad 1 \leq j \leq n .
$$

It should be observed that, in either case, Theorem 4 and (15) hold for every $q \in$ $(1, \infty)$, although $A_{(q)}$ depends on $q$, of course.

(b) It follows from (15) that $\lambda_{0}(A)$ is well-defined.

We also set

$$
W_{q, \mathcal{B}}^{1}:=W_{q,(1-\chi) \gamma}^{1}:=\left\{u \in W_{q}^{1} ;(1-\chi) \gamma u=0\right\} .
$$

Note that

$$
W_{q^{\prime}, \mathcal{B}^{\sharp}}^{1}=W_{q^{\prime},(1-\chi) \gamma}^{1} .
$$

In the following, $(\mathcal{A}, \mathcal{B})$ is said to satisfy the very weak maximum principle (in $\left.L_{q}\right)$ if, given any $u$ such that

$$
u \in L_{q}, \quad\left\langle\mathcal{A}^{\sharp} v, u\right\rangle \geq 0 \text { for all } v \in\left(W_{q^{\prime}, \mathcal{B}^{\sharp}}^{2}\right)^{+},
$$

it follows that $u \geq 0$. It satisfies the weak maximum principle (in $W_{q}^{1}$ ) if it is a consequence of

$$
u \in W_{q}^{1}, \quad \mathfrak{a}(v, u) \geq 0 \text { for all } v \in\left(W_{q^{\prime},(1-\chi) \gamma}^{1}\right)^{+}, \quad(1-\chi) \gamma u \geq 0
$$

that $u \geq 0$. Lastly, $(\mathcal{A}, \mathcal{B})$ satisfies the maximum principle or is inverse positive (on $W_{q}^{2}$ ) if

$$
u \in W_{q}^{2}, \quad \mathcal{A} u \geq 0 \text { in } \Omega, \quad \mathcal{B} u \geq 0 \text { on } \Gamma
$$

imply $u \geq 0$.

\section{Theorem 6.}

(1) Consider the following assertions:

(i) $(\mathcal{A}, \mathcal{B})$ satisfies the very weak maximum principle in $L_{q}$.

(ii) $(\mathcal{A}, \mathcal{B})$ satisfies the weak maximum principle in $W_{q}^{1}$.

(iii) $(\mathcal{A}, \mathcal{B})$ is inverse positive on $W_{q}^{2}$. 
Then (i) implies (ii), and (ii) implies (iii). If, in addition, $A$ is surjective then all these assertions are equivalent.

(2) If $\lambda_{0}:=\lambda_{0}(A)>0$ then $(\mathcal{A}, \mathcal{B})$ is inverse positive. Conversely, if $(\mathcal{A}, \mathcal{B})$ is inverse positive and $A$ is surjective then $\lambda_{0}>0$.

(3) The semigroup $\mathcal{U}_{A}$ is positive.

(4) If $\sigma(A) \neq \varnothing$ then $\lambda_{0} \in \sigma(A)$.

The proof of this theorem is given in Section 16.

\section{Remarks 7.}

(a) Although our regularity assumptions guarantee that (15) holds for every $q \in(1, \infty)$, we do not know whether $\lambda_{0}$ is independent of $q$. This would follow from the spectral invariance of elliptic operators. However, the known results (see [13], [31], [45], [50], [70]) do not seem to apply to the present situation. It is also not known whether $\sigma(A) \neq \varnothing$, in general.

(b) Suppose that only the weaker assumption (16) is satisfied. Then Theorem 6 remains valid, provided we omit assertion (i) in (1).

We emphasize the fact that the results of this section are true under the mere assumption that $\Omega$ has a compact boundary. We are not aware of any related theorem valid for the case of exterior domains.

\section{Nonhomogeneous problems}

Of course, the validity of a maximum principle has implications on the solvability of nonhomogeneous elliptic boundary value problems. This is made precise in the present section.

We put

$$
W_{q, \mathcal{B}}^{-2}:=\left(W_{q^{\prime}, \mathcal{B}}^{2}\right)^{\prime}, \quad W_{q, \mathcal{B}}^{-1}:=W_{q,(1-\chi) \gamma}^{-1}:=\left(W_{q^{\prime},(1-\chi) \gamma}^{1}\right)^{\prime}
$$

with respect to the duality pairings $\langle\cdot, \cdot\rangle$ naturally induced by $(10)$. It follows that

$$
W_{q, \mathcal{B}}^{2} \stackrel{d}{\hookrightarrow} W_{q, \mathcal{B}}^{1} \stackrel{d}{\hookrightarrow} L_{q} \stackrel{d}{\hookrightarrow} W_{q, \mathcal{B}}^{-1} \stackrel{d}{\hookrightarrow} W_{q, \mathcal{B}}^{-2} .
$$

We endow $W_{q, \mathcal{B}}^{-k}$ for $k \in\{1,2\}$ with the natural dual order whose positive cone is the dual of the cone $\left(W_{q^{\prime}, \mathcal{B}^{\sharp}}^{k}\right)^{+}$. Then $W_{q, \mathcal{B}}^{-1}$ and $W_{q, \mathcal{B}}^{-2}$ are $O B S s$ and each one of the injection maps in (19) is positive.

Next we consider the nonhomogeneous boundary value problem

$$
\mathcal{A} u=f \text { in } \Omega, \quad \mathcal{B} u=g \text { on } \Gamma \text {. }
$$

A (strong) $W_{q}^{2}$ solution is a $u \in W_{q}^{2}$ with

$$
(\mathcal{A} u, \mathcal{B} u)=(f, g) \quad \text { in } L_{q} \times \partial^{2} W_{q} .
$$


By a (weak) $W_{q}^{1}$ solution we mean a $u \in W_{q}^{1}$ satisfying

$$
\left.\begin{array}{rlrl}
\mathfrak{a}(v, u) & =\langle v, f\rangle & & \text { for } v \in W_{q^{\prime},(1-\chi) \gamma}^{1}, \\
1-\chi) \gamma u & =(1-\chi) g & & \text { on } \Gamma .
\end{array}\right\}
$$

Lastly, $u$ is said to be a (very weak) $L_{q}$ solution of (20) if $u \in L_{q}$ and

$$
\left\langle\mathcal{A}^{\sharp} v, u\right\rangle=\langle v, f\rangle+\left\langle\partial_{\mu} v,(\chi-1) g\right\rangle_{\Gamma}+\langle\gamma v, \chi g\rangle_{\Gamma}
$$

for $v \in W_{q^{\prime}, \mathcal{B}^{\sharp}}^{2}$.

The following theorem gives a further characterization of the positivity of $\lambda_{0}$.

Theorem 8. The following are equivalent:

(i) $\lambda_{0}>0$.

(ii) Problem (20) has for each $(f, g) \in\left(L_{q} \times \partial W_{q}^{2}\right)^{+}$a unique nonnegative $W_{q}^{2}$ solution.

(iii) Problem (20) has for each $(f, g) \in\left(W_{q, \mathcal{B}}^{-1} \times \partial W_{q}^{1}\right)^{+}$a unique nonnegative $W_{q}^{1}$ solution.

(iv) Problem (20) has for each $(f, g) \in\left(W_{q, \mathcal{B}}^{-2} \times \partial W_{q}^{0}\right)^{+}$a unique nonnegative $L_{q}$ solution.

The proof of this theorem is also given in Section 16.

Remark 9. If we presuppose only the weaker hypothesis (16) then Theorem 8 remains valid if assertion (iv) is omitted.

\section{The principal eigenvalue}

Throughout this section we suppose that $\Omega$ is bounded. Then we can considerably improve on the results of the preceding section. For this we put

$$
W_{\infty-}^{2}:=\bigcap_{1<q<\infty} W_{q}^{2} .
$$

We also assume that only the weaker hypothesis (16) is satisfied.

Theorem 10. Suppose that $\Omega$ is bounded. Then A has a compact resolvent. Hence $\sigma(A)$ is discrete and each $\lambda \in \sigma(A)$ is an eigenvalue of finite multiplicity. Moreover,

$$
N_{A}(\lambda) \subset W_{\infty-}^{2}
$$

for $\lambda \in \sigma(A)$. 
This theorem, whose proof is found in Section 17, shows, in particular, that the spectrum, the eigenspaces, and the generalized eigenspaces are independent of $q \in(1, \infty)$. Thus we say that $\lambda$ is an eigenvalue of $(\mathcal{A}, \mathcal{B})$ iff there exists $u \in W_{\infty-}^{2} \backslash\{0\}$ satisfying

$$
\mathcal{A} u=\lambda u \text { in } \Omega, \quad \mathcal{B} u=0 \text { on } \Gamma .
$$

The multiplicity of $\lambda$ is, by definition, the multiplicity of $\lambda$ as an eigenvalue of the $L_{q}$ realization of $(\mathcal{A}, \mathcal{B})$ for some $q \in(1, \infty)$. Thanks to Theorem 10 this definition is independent of $q$. Thus it makes sense to say that $\sigma(A)$ is the spectrum of $(\mathcal{A}, \mathcal{B})$, that is, $\sigma(\mathcal{A}, \mathcal{B}):=\sigma(A)$. If $\sigma(\mathcal{A}, \mathcal{B}) \neq \varnothing$ then it follows from Theorem 6 that $\lambda_{0}$ is the smallest eigenvalue of $(\mathcal{A}, \mathcal{B})$, the principal eigenvalue, also denoted by $\lambda_{0}(\mathcal{A}, \mathcal{B})$, or, more precisely, by $\lambda_{0}(\mathcal{A}, \mathcal{B}, \Omega)$. The following theorem, whose proof is also given in Section 17, guarantees its existence.

Theorem 11. If $\Omega$ is bounded then $\sigma(\mathcal{A}, \mathcal{B}) \neq \varnothing$, and the principal eigenvalue has a positive eigenfunction. Furthermore, $\lambda_{0}>0$ iff $(\mathcal{A}, \mathcal{B})$ is inverse positive.

In general, $\lambda_{0}$ is not the only eigenvalue with a positive eigenfunction. This follows immediately by considering diagonal (that is, uncoupled) systems. Thus, in order to guarantee that $\lambda_{0}$ is the only eigenvalue with a positive eigenfunction we have to ascertain that the coupling is sufficiently strong. For this we need some preparation.

Let $R$ be a ring with unit. Then $\pi \in R^{N \times N}$ is a permutation matrix iff it contains exactly one unit in every row and every column, and zeros elsewhere. The matrix $\alpha \in R^{N \times N}$ is reducible iff there exist $M \in\{1, \ldots, N-1\}$ and a permutation matrix $\pi$ such that

$$
\pi \alpha \pi^{\top}=\left[\begin{array}{ll}
\alpha_{11} & \alpha_{12} \\
0 & \alpha_{22}
\end{array}\right]
$$

with $\alpha_{11} \in R^{M \times M}$, and $\alpha$ is irreducible otherwise. It is well-known and not difficult to see that $\alpha$ is irreducible iff, given $r, s \in\{1, \ldots, N\}$, there exist indices $s_{\rho} \in\{1, \ldots, N\}$, $0 \leq \rho \leq k$, with $s_{0}=s$ and $s_{k}=r$ such that $\alpha^{\xi \eta} \neq 0$ for $(\xi, \eta)$ belonging to $\left\{\left(s_{1}, s_{0}\right),\left(s_{2}, s_{1}\right), \ldots,\left(s_{k}, s_{k-1}\right)\right\}$.

Let $R_{1}$ and $R_{2}$ be rings with unit $1_{1}$ and $1_{2}$, respectively. Then $R_{1} \times R_{2}$ is a ring, the product ring, with multiplication being defined component-wise. It has a unit, namely $\left(1_{1}, 1_{2}\right)$. Suppose that $\alpha_{i} \in R_{i}^{N \times N}$ for $i=1,2$. Then we denote by

$$
\left[\left(\alpha_{1}, \alpha_{2}\right)\right] \in\left(R_{1} \times R_{2}\right)^{N \times N}
$$

the matrix whose entry at position $(r, s)$ equals $\left(\alpha_{1}^{r s}, \alpha_{2}^{r s}\right) \in R_{1} \times R_{2}$.

Note that $L_{\infty}(\Omega, \mathbb{R})$ and $C^{1-}(\Gamma, \mathbb{R})$ are rings with unit $\mathbf{1}_{\Omega}$ and $\mathbf{1}_{\Gamma}$, respectively, where, given any nonempty set $X$, we denote by $\mathbf{1}_{X}$ the constant map $x \mapsto 1$. We also set

$$
a^{\triangle}:=\operatorname{diag}\left[a^{11}, \ldots, a^{N N}\right], \quad b^{\triangle}:=\operatorname{diag}\left[b^{11}, \ldots, b^{N N}\right]
$$

and

$$
a^{\bullet}:=a^{\triangle}-a, \quad b^{\bullet}:=b^{\triangle}-b .
$$


Observe that $a^{\bullet}$ and $b^{\bullet}$ are nonnegative and have zeros in the diagonals.

Suppose that $N \geq 2$. Then the pair $(a, b)$, more precisely: $(a, \chi b \chi)$, is said to be irreducible iff

$$
\left[\left(a^{\bullet}, \chi b^{\bullet} \chi\right)\right] \in\left(L_{\infty}(\Omega, \mathbb{R}) \times C^{1-}(\Gamma, \mathbb{R})\right)^{N \times N}
$$

is irreducible. Thus $(a, b)$ is irreducible iff, given any $r, s \in\{1, \ldots, N\}$, there exist $s_{\rho} \in$ $\{1, \ldots, N\}, 0 \leq \rho \leq k$, with $s_{0}=s$ and $s_{k}=r$ such that

$$
\text { either } \chi^{\xi} b^{\xi \eta} \chi^{\eta}>0 \text { or } a^{\xi \eta}>0
$$

for

$$
(\xi, \eta) \in\left\{\left(s_{1}, s_{0}\right),\left(s_{2}, s_{1}\right), \ldots,\left(s_{k}, s_{k-1}\right)\right\} .
$$

If this is the case, we also say that $(\mathcal{A}, \mathcal{B})$ is irreducible.

A function $u \in L_{1, \text { loc }}$ is strongly positive if $u \in C^{1}(\bar{\Omega})$ and satisfies for each $r \in$ $\{1, \ldots, N\}$ the inequalities $u^{r}(x)>0$ for $x \in \Omega \cup \Gamma_{1}^{r}$ and $\partial_{\mu^{r}} u^{r}(y)<0$ for $y \in \Gamma_{0}^{r}$ with $u^{r}(y)=0$.

The next theorem is the basis for a detailed study of the principal eigenvalue. Its proof is found in Section 17.

Theorem 12. Let $\Omega$ be bounded and suppose that either $N=1$ or $(a, b)$ is irreducible. Then the principal eigenvalue is simple and has a strongly positive eigenfunction. It is the only eigenvalue with a positive eigenfunction, and every other eigenvalue satisfies

$$
\operatorname{Re} \lambda>\lambda_{0} \text {. }
$$

In the scalar case this theorem is well-known and has first been proved, in the case of general boundary conditions and without a positivity restriction for $b$, in [3]. That proof contained a gap since it had not been asserted that the spectrum is nonempty. Motivated by this, de Pagter [33] derived a general theorem on irreducible compact positive operators on Banach lattices implying that such an operator has a strictly positive spectral radius. That theorem can be used to fill the gap (cf. the proof of [11, Theorem 2.2]). It has also been employed in papers by Sweers and coauthors (cf. [20], [71]) in the case of irreducible cooperative elliptic systems with Dirichlet boundary conditions to prove essentially Theorem 12 (under more restrictive regularity hypotheses). The proof given in Section 17 is much more elementary. It is solely based on the classical Krein-Rutman theorem and does not invoke the rather deep de Pagter result. The case of Dirichlet boundary conditions has also been considered in [56], but under the much stronger assumption that $a^{r s}(x)>0$ for $r \neq s$ and all $x \in \Omega$, where $a$ is supposed to be continuous.

\section{The strong maximum principle}

The boundary value problem $(\mathcal{A}, \mathcal{B})$ is said to satisfy the strong maximum principle if

$$
q>n, \quad u \in W_{q}^{2} \backslash\{0\}, \quad \mathcal{A} u \geq 0 \text { in } \Omega, \quad \mathcal{B} u \geq 0 \text { on } \Gamma
$$


imply that $u$ is strongly positive.

We say that $u$ is a strict $W_{q}^{2}$ supersolution for $(\mathcal{A}, \mathcal{B})$ if $u$ belongs to $W_{q}^{2}$ and

$$
(\mathcal{A} u, \mathcal{B} u)>0 .
$$

It is a strict $W_{q}^{1}$ supersolution for $(\mathcal{A}, \mathcal{B})$ if it satisfies (18) with a strict inequality sign either in the first place for at least one $v$ or in the second place. Finally, $u$ is a strict $L_{q}$ supersolution for $(\mathcal{A}, \mathcal{B})$ if it satisfies (17) with a strict inequality sign for at least one $v$. It follows that each $W_{q}^{2}$ supersolution is a $W_{q}^{1}$ supersolution, and each $W_{q}^{1}$ supersolution is an $L_{q}$ supersolution.

Using these concepts the following characterizations of the maximum principles are valid.

Theorem 13. Let $\Omega$ be bounded and suppose that either $N=1$ or $(a, b)$ is irreducible. Then the following are equivalent:

(i) $\lambda_{0}>0$.

(ii) $(\mathcal{A}, \mathcal{B})$ satisfies the very weak maximum principle.

(iii) $(\mathcal{A}, \mathcal{B})$ satisfies the weak maximum principle.

(iv) $(\mathcal{A}, \mathcal{B})$ satisfies the strong maximum principle.

(v) $(\mathcal{A}, \mathcal{B})$ possesses a positive strict $L_{q}$ supersolution.

Remark 14. Assume that only condition (16) is satisfied. Then Theorem 13 remains valid, provided the following modifications are implemented: assertion (ii) is omitted and ' $L_{q}$ supersolution' in (v) is replaced by ' $W_{q}^{1}$ supersolution'.

Given the assumptions of the previous theorem, we can also improve on the solvability statements of Theorem 8. For this, $u \in L_{q}$ is said to be strictly positive if $u^{r}(x)>0$ for a.a. $x \in \Omega$ and each $r \in\{1, \ldots, N\}$.

Theorem 15. Suppose that $\Omega$ is bounded and that either $N=1$ or $(a, b)$ is irreducible. If $\lambda_{0}>0$ then, given any $(f, g) \in\left(L_{q} \times \partial W_{q}^{2}\right)^{+} \backslash\{0\}$, the unique positive solution of (20) is strictly positive. If $q>n$ then it is strongly positive.

The important new ingredient distinguishing Theorem 13 from Theorem 6 is the characterization of the validity of the maximum principle through the existence of a positive strict supersolution. In the scalar case $N=1$ and in the framework of classical solutions, Protter and Weinberger seem to be the first to observe that the existence of a strict classical supersolution

$$
w \in C^{2}(\Omega) \cap C^{1}\left(\Omega \cup \Gamma_{1}\right) \cap C(\bar{\Omega})
$$

satisfying

$$
w(x)>0 \text { for all } x \in \bar{\Omega}
$$


implies the existence of the maximum principle for $(\mathcal{A}, \mathcal{B})$ (cf. [63, Section II.5]). In the usual weak $\stackrel{\circ}{H}^{1}$ setting and with weak regularity assumptions it has also been shown in [23] that the existence of a positive $H^{1}$ supersolution characterizes the weak maximum principle for the Dirichlet problem. The fact that (i) and (iv) of Theorem 13 as well as the existence of a positive strict classical supersolution $w$, such that $w_{r}(x)>0$ for all $x \in \bar{\Omega}$ and $1 \leq r \leq N$, are equivalent has first been observed in [56] in the case of Dirichlet problems for cooperative elliptic systems satisfying the strong irreducibility condition explained in the preceding section. In the scalar case, López-Gómez [52] could then relax the hypotheses on a classical strict supersolution $w$ by requiring $w(y) \geq 0$ for $y$ in $\Gamma$, keeping the assumption $w(x)>0$ for $x \in \Omega$. This result has been extended to the case of a general boundary operator in [11, Theorem 2.4], where positive strict $W_{q}^{2}$ supersolutions with $q>n$ are being considered. The proof in [11] relies on [3, Theorem 6.1] which, in turn, is a consequence of the Protter-Weinberger result cited above and a construction of a strict $W_{q}^{2}$ supersolution $w$ for $q>n$ satisfying $w(x)>0$ for all $x \in \bar{\Omega}$ (see [3, Lemma 5.1]), The latter construction is somewhat involved and complicated (but see Remark 36(a)). This prompted López-Gómez [55] to give a simpler proof of Theorem 2.4 in [11] in the framework of $C^{2+\alpha}$ solutions by extending a version of the maximum principle due to Walter [76]. It should be remarked that in none of those results $(\mathcal{A}, \mathcal{B})$ is required to possess divergence form.

The fact that a strict positive supersolution in the class $W_{n}^{2}\left(\Omega, \mathbb{R}^{N}\right) \cap C\left(\bar{\Omega}, \mathbb{R}^{N}\right)$ implies the maximum principle and the existence of a unique positive eigenfunction is the main theorem in [71] for the Dirichlet problem of irreducible cooperative systems (also see [20]).

Our Theorem 13 is much more general since it applies to systems with general coupled boundary conditions and replaces $W_{q}^{2}$ supersolutions for $q \geq n$ by the much weaker concept of $L_{q}$ supersolutions.

The importance of the results of this section is seen from the theorems in Sections 8-11. Furthermore, it should be noted that the results of Theorems 13 and 15 suffice to apply the abstract techniques of [1] to irreducible cooperative elliptic systems. By this way one obtains extensions of the existence, multiplicity, and bifurcation results contained in [1]. For example, one can extend the three solutions theorem [1, Theorem 14.2] to such systems, etc. We leave the details to interested readers.

It should also be remarked that, thanks to Theorems 12 and 15 (and their proofs), it is not difficult to extend the anti-maximum principle of Clément and Peletier [26] (also see [72]) to cooperative irreducible systems of the form $(\mathcal{A}, \mathcal{B})$. Details are also left to the readers.

\section{Monotonicity of the principal eigenvalue}

Throughout this section $\Omega$ is again bounded and only the weaker hypothesis (16) is imposed.

We discuss monotonicity properties of the principal eigenvalue with respect to variations 
of $a, b, \Omega$, and the boundary conditions.

First we suppose that $\widetilde{a} \in L_{\infty}\left(\Omega, \mathbb{R}^{N \times N}\right)$ and $\widetilde{b} \in C^{1-}\left(\Gamma, \mathbb{R}^{N \times N}\right)$ are cooperative. Then we define $(\widetilde{\mathcal{A}}, \widetilde{\mathcal{B}})$ by replacing $a$ and $b$ in (8) and (9) by $\widetilde{a}$ and $\widetilde{b}$, respectively.

Theorem 16. Suppose that either $N=1$ or $(a, b)$ and $(\widetilde{a}, \widetilde{b})$ are irreducible. If

$$
(a, \chi b \chi)<(\widetilde{a}, \chi \widetilde{b} \chi)
$$

then

$$
\lambda_{0}(\mathcal{A}, \mathcal{B})<\lambda_{0}(\widetilde{\mathcal{A}}, \widetilde{\mathcal{B}})
$$

Proof. Let $u_{0}$ be a positive eigenfunction of $(\mathcal{A}, \mathcal{B})$ to the eigenvalue

$$
\lambda_{0}:=\lambda_{0}(\mathcal{A}, \mathcal{B}) \text {. }
$$

Then

$$
\left(\widetilde{\mathcal{A}}-\lambda_{0}\right) u_{0}=\left(\mathcal{A}-\lambda_{0}\right) u_{0}+(\widetilde{a}-a) u_{0}=(\widetilde{a}-a) u_{0} \quad \text { in } \Omega
$$

and

$$
\widetilde{\mathcal{B}} u_{0}=\mathcal{B} u_{0}+\chi(\widetilde{b}-b) \chi \gamma u_{0}=\chi(\widetilde{b}-b) \chi \gamma u_{0} \quad \text { on } \Gamma .
$$

Hence it follows from (27) and the strong positivity of $u_{0}$, guaranteed by Theorem 12 , that $u_{0}$ is a positive strict $W_{q}^{2}$ supersolution for $\left(\widetilde{\mathcal{A}}-\lambda_{0}, \mathcal{B}\right)$. Thus

$$
0<\lambda_{0}\left(\widetilde{\mathcal{A}}-\lambda_{0}, \widetilde{\mathcal{B}}\right)=\lambda_{0}(\widetilde{\mathcal{A}}, \widetilde{\mathcal{B}})-\lambda_{0}
$$

by Theorem 13 and Remark 14.

The next theorem shows, in particular, that the principle eigenvalue decreases strictly if a Dirichlet boundary condition is replaced for at least one component of $u$ by a Neumann boundary condition on at least one component of $\Gamma$. To make this precise we suppose that $\widehat{\chi} \in C\left(\Gamma, \mathbb{R}_{\mathrm{diag}}^{N \times N}\right)$ is a boundary identification map. Then we put

$$
\widehat{\mathcal{B}} u:=\widehat{\chi}\left(\partial_{\mu} u+b u\right)+(1-\widehat{\chi}) u \text {. }
$$

Note that $\chi<\widehat{\chi}$ means that $\Gamma_{0}^{r} \supset \widehat{\Gamma}_{0}^{r}$ for $1 \leq r \leq N$ where at least one of the inclusions is proper.

Theorem 17. Suppose that either $N=1$ or $(a, \chi b \chi)$ is irreducible. Also suppose that

$$
\chi<\hat{\chi} \text { and }(\widehat{\chi}-\chi) b^{\triangle} \leq 0 .
$$

Then

$$
\lambda_{0}(\mathcal{A}, \mathcal{B})>\lambda_{0}(\mathcal{A}, \widehat{\mathcal{B}})
$$

Proof. First observe that the irreducibility of $(a, \chi b \chi)$ and $\chi<\hat{\chi}$ imply the one of $(a, \widehat{\chi} b \widehat{\chi})$. Thus, by Theorem 12 , there exists a strongly positive eigenfunction $\widehat{u}_{0}$ of $(\mathcal{A}, \widehat{\mathcal{B}})$ to the eigenvalue

$$
\widehat{\lambda}_{0}:=\lambda_{0}(\mathcal{A}, \widehat{\mathcal{B}}) \text {. }
$$


Thus, given $v \in\left(W_{q^{\prime},(1-\chi) \gamma}^{1}\right)^{+}$,

$$
\mathfrak{a}\left(v, \widehat{u}_{0}\right)-\widehat{\lambda}_{0}\left\langle v, \widehat{u}_{0}\right\rangle=\widehat{\mathfrak{a}}\left(v, \widehat{u}_{0}\right)-\widehat{\lambda}_{0}\left\langle v, \widehat{u}_{0}\right\rangle+\left\langle\gamma v,(\chi b-\widehat{\chi} b) \gamma \widehat{u}_{0}\right\rangle_{\Gamma}
$$

where $\widehat{\mathfrak{a}}$ is the Dirichlet form of $(\mathcal{A}, \widehat{\mathcal{B}})$. Since $W_{q^{\prime},(1-\chi) \gamma}^{1} \subset W_{q^{\prime},(1-\widehat{\chi}) \gamma}^{1}$, thanks to $\chi<\widehat{\chi}$, it follows that

$$
\widehat{\mathfrak{a}}\left(v, \widehat{u}_{0}\right)-\widehat{\lambda}_{0}\left\langle v, \widehat{u}_{0}\right\rangle=0, \quad v \in\left(W_{q^{\prime},(1-\chi) \gamma}^{1}\right)^{+} .
$$

Also observe that

$$
\left\langle\gamma v,(\chi-\widehat{\chi}) b \gamma \widehat{u}_{0}\right\rangle_{\Gamma} \geq-\left\langle\gamma v,(\widehat{\chi}-\chi) b^{\triangle} \gamma u_{0}\right\rangle_{\Gamma}
$$

since $-b^{r s} \geq 0$ for $r \neq s$. Hence we deduce from (28) that

$$
\mathfrak{a}\left(v, \widehat{u}_{0}\right)-\widehat{\lambda}_{0}\left\langle v, \widehat{u}_{0}\right\rangle \geq 0, \quad v \in\left(W_{q^{\prime},(1-\chi) \gamma}^{1}\right)^{+} .
$$

From $\chi<\widehat{\chi}$ and the strong positivity of $\widehat{u}_{0}$ we also infer that

$$
(1-\chi) \gamma \widehat{u}_{0}>0 \quad \text { on } \Gamma \text {. }
$$

Thus $\widehat{u}_{0}$ is a strict $W_{q}^{1}$ supersolution for $\left(\mathcal{A}-\widehat{\lambda}_{0}, \mathcal{B}\right)$. Hence

$$
0<\lambda_{0}\left(\mathcal{A}-\widehat{\lambda}_{0}, \mathcal{B}\right)=\lambda_{0}(\mathcal{A}, \mathcal{B})-\widehat{\lambda}_{0}
$$

by Theorem 13 and Remark 14 .

Note that (28) implies that $b^{r r} \leq 0$ on each component of $\Gamma$ on which a Dirichlet boundary condition is replaced by a Robin one.

For example, it follows from Theorem 17 that, given that $a$ is irreducible if $N>1$, the principal eigenvalues for the pure Neumann, the general Robin, and the pure Dirichlet condition satisfy

$$
\lambda_{0}\left(\mathcal{A}, \partial_{\mu}\right)<\lambda_{0}(\mathcal{A}, \mathcal{B})<\lambda_{0}(\mathcal{A}, \gamma)
$$

provided $b^{r r} \leq 0$ for $1 \leq r \leq N$ with at least one strict inequality sign. Also note that, thanks to the cooperativity assumption, the second inequality in this chain is consistent with Theorem 16.

Our next theorem shows that the principle eigenvalue increases if the domain shrinks and if on each boundary component being moved inside the original boundary condition is replaced by a Dirichlet one.

Theorem 18. Let $\Omega^{\star}$ be a proper $C^{2}$ subdomain of $\Omega$ with boundary $\Gamma^{\star}$. Denote by $\Sigma$ the union of all components of $\Gamma^{\star}$ having a nonempty intersection with $\Omega$, and put

$$
\Sigma^{\prime}:=\Gamma^{\star} \backslash \Sigma \text {. }
$$

Define a boundary identification map $\chi^{\star} \in C\left(\Gamma^{\star}, \mathbb{R}_{\mathrm{diag}}^{N \times N}\right)$ for $\Omega^{\star}$ by

$$
\chi^{\star}\left|\Sigma:=0, \quad \chi^{\star}\right| \Sigma^{\prime}:=\chi \mid \Sigma^{\prime}
$$

and put

$$
\left(\left[a_{j k}^{*}\right],\left(a_{1}^{*}, \ldots, a_{n}^{*}\right), a^{*}\right):=\left(\left[a_{j k}\right],\left(a_{1}, \ldots, a_{n}\right), a\right) \mid \Omega^{*}
$$


and

$$
\mathcal{A}^{\star} u:=\mathcal{A} u, \quad \mathcal{B}^{\star} u:=\chi^{\star}\left(\partial_{\mu^{\star}} u+b u\right)+\left(1-\chi^{\star}\right) u
$$

for $u \in W_{q}^{2}\left(\Omega^{\star}, \mathbb{R}^{N}\right)$. Then

$$
\lambda_{0}(\mathcal{A}, \mathcal{B}, \Omega)<\lambda_{0}\left(\mathcal{A}^{\star}, \mathcal{B}^{\star}, \Omega^{\star}\right)
$$

provided $\left(a^{\star}, \chi^{\star} b \chi^{\star}\right)$ is irreducible if $N>1$.

Proof. Observe that the irreducibility of $\left(a^{\star}, \chi^{\star} b \chi^{\star}\right)$ implies the one of $(a, \chi b \chi)$. Also note that the strong positivity of a positive eigenfunction $u$ of $(\mathcal{A}, \mathcal{B})$ to the eigenvalue $\lambda_{0}:=\lambda_{0}(\mathcal{A}, \mathcal{B}, \Omega)$ implies that $u \mid \Sigma>0$. Hence $u$ is a positive strict $W_{q}^{2}$ supersolution for $\left(\mathcal{A}^{\star}-\lambda_{0}, \mathcal{B}^{\star}, \Omega^{\star}\right)$. Now the assertion follows once more from Theorem 13 and Remark 14.

The theorems of this section, as well as their proofs, are more or less straightforward extensions and sharpenings of corresponding results established in the scalar case (i.e., $N=1$ ) by López-Gómez [52] for Dirichlet and by Cano-Casanova and López-Gómez [21] for general boundary conditions (also see [37] and, for the Dirichlet problem in a weak setting, [24]). In the particular case of Theorem 16 where $\chi=0$ and $\tilde{\chi}=0$, that is, for Dirichlet problems, the monotonicity of $\lambda_{0}$ as a function of $a$ has also been shown in [56], given the much more restrictive assumption that $a, \widetilde{a} \in C\left(\bar{\Omega}, \mathbb{R}^{N \times N}\right)$ and satisfy $\widetilde{a} \geq a$ and $\widetilde{a}^{r s}\left(x_{0}\right)>a^{r s}\left(x_{0}\right)$ for some $x_{0} \in \Omega$ and all $r, s \in\{1, \ldots, N\}$.

The weak Dirichlet problem in $W_{2, \gamma}^{1}$ for scalar equations has attracted a lot of interest. In this case general perturbation theorems are due to Arendt and coauthors [14], [17], Stollmann [69] and, in particular, Daners [28], [29], who has perhaps the most general results. For the weak Robin boundary value problem in the scalar case we refer to [27].

\section{Continuity of the principal eigenvalue}

We suppose again that $\Omega$ is bounded and put

$$
\begin{aligned}
\mathbb{E}^{1}(\Omega):=C^{1}\left(\bar{\Omega}, \mathbb{R}_{\text {diag }}^{N \times N}\right)^{n \times n} \times L_{\infty}\left(\Omega, \mathbb{R}_{\text {diag }}^{N \times N}\right)^{n} & \times L_{\infty}\left(\Omega, \mathbb{R}^{N \times N}\right) \\
& \times C^{1-}\left(\Gamma, \mathbb{R}^{N \times N}\right) \times C\left(\Gamma, \mathbb{R}_{\text {diag }}^{N \times N}\right) .
\end{aligned}
$$

Given

$$
\alpha:=\left(\left[a_{j k}\right],\left(a_{1}, \ldots, a_{n}\right), a, b, \chi\right) \in \mathbb{E}^{1}(\Omega),
$$

we define $(\mathcal{A}(\alpha), \mathcal{B}(\alpha))$ on $\Omega$ by (8) and (9). Then we denote by $\mathcal{E}(\Omega)$ the set of all $\alpha \in \mathbb{E}^{1}(\Omega)$ such that $\chi$ is a boundary characterization map for $\Omega$ and $(\mathcal{A}(\alpha), \mathcal{B}(\alpha))$ is a cooperative elliptic boundary value problem on $\Omega$ such that $(a, b)$ is irreducible if $N>1$.

Let $\underset{\sim}{\Omega}$ be a bounded $C^{2}$ domain in $\mathbb{R}^{n}$ and let $\varphi: \bar{\Omega} \rightarrow \underset{\sim}{\bar{\Omega}}$ be a $C^{2}$ diffeomorphism. Then $\varphi_{\partial}:=\varphi \mid \Gamma$ is a $C^{2}$ diffeomorphism from $\Gamma$ onto $\partial \underset{\sim}{\Omega}$. Given

$$
\underset{\sim}{\alpha}:=\left([\underset{\sim}{a} j k],\left(\underset{\sim}{a_{1}}, \ldots, \underset{\sim}{a}\right), \underset{\sim}{a}, \underset{\sim}{b}, \underset{\sim}{\chi}\right) \in \mathbb{E}^{1}(\underset{\sim}{\Omega}),
$$


we put

$$
\varphi^{*} \underset{\sim}{\alpha}:=\left(\left[\varphi^{*} \underset{\sim}{a} j k\right],\left(\varphi^{*} \underset{\sim}{a_{1}}, \ldots, \varphi^{*} \underset{\sim}{a}\right), \varphi^{*} \underset{\sim}{a}, \varphi_{\partial}^{*} \underset{\sim}{b}, \varphi_{\partial}^{*} \underset{\sim}{\chi}\right) \in \mathbb{E}^{1}(\Omega),
$$

where, for a function $\underset{\sim}{f}$ on $\underset{\sim}{\Omega}$, the pull back of $\underset{\sim}{f}$ by $\varphi$ is defined by

$$
\varphi^{*} \underset{\sim}{f}:=\underset{\sim}{f} \circ \varphi
$$

Note that $\varphi^{*} \underset{\sim}{\alpha} \in \mathcal{E}(\Omega)$ if $\underset{\sim}{\alpha} \in \mathcal{E}(\underset{\sim}{\Omega})$.

Let $\left(\Omega_{i}\right)$ be a sequence of bounded $C^{2}$ domains in $\mathbb{R}^{n}$. Then it is said to be $C^{1}$ converging towards $\Omega$ if there exist orientation preserving $C^{2}$ diffeomorphisms $\varphi_{i}: \bar{\Omega} \rightarrow \bar{\Omega}_{i}$ such that $\varphi_{i} \rightarrow \mathrm{id}_{\bar{\Omega}}$ in $C^{1}\left(\bar{\Omega}, \mathbb{R}^{N}\right)$. Such a sequence $\left(\varphi_{i}\right)$ is said to be a representation sequence for $\left(\Omega_{i}\right)$.

Sequences of bounded $C^{2}$ domains being $C^{1}$ convergent towards $\Omega$ are often obtained by deformations of $\Omega$ be means of sequences of transformation groups for $\bar{\Omega}$. This is true, in particular, if the transformation group is generated by a $C^{2}$ vector field.

Example 19. Suppose that $\Omega$ is bounded and $f \in C^{2}\left(\bar{\Omega}, \mathbb{R}^{n}\right)$. For each $x \in \bar{\Omega}$ let $\varphi(\tau, x)$ be the solution at time $\tau$ of the initial value problem

$$
\dot{\xi}=f(\xi), \quad \xi(0)=x
$$

Then there exist $\tau^{-}<0<\tau^{+}$such that

$$
\varphi \in C^{2}\left(\left(\tau^{-}, \tau^{+}\right) \times \bar{\Omega}, \mathbb{R}^{n}\right)
$$

and $\varphi^{\tau}:=\varphi(\tau, \cdot)$ is for each $\tau \in\left(\tau^{-}, \tau^{+}\right)$an orientation preserving $C^{2}$ diffeomorphism from $\bar{\Omega}$ onto $\bar{\Omega}_{\tau}:=\varphi^{\tau}(\bar{\Omega})$. Thus $\Omega_{\tau}:=\varphi^{\tau}(\Omega)$ is a $C^{2}$ domain in $\mathbb{R}^{n}$ and, given any sequence $\left(\tau_{j}\right)$ in $\left(\tau^{-}, \tau^{+}\right)$with $\tau_{j} \rightarrow 0$, the sequence $\left(\Omega_{\tau_{j}}\right)$ is $C^{1}$ converging (in fact: $C^{2}$ converging) towards $\Omega$, and $\left(\varphi_{\tau_{j}}\right)$ is a representation sequence for $\left(\Omega_{\tau_{j}}\right)$.

Proof. The theory of ordinary differential equations (e.g., [6, Section 10]) implies these assertions.

Now we fix $r, s, t \in[1, \infty]$ satisfying

$$
(r, s, t)= \begin{cases}(n, n / 2, n-1) & \text { if } \quad n \geq 3, \\ \in(2, \infty] \times(1, \infty] \times(1, \infty] & \text { if } \quad n=2, \\ (1,1,1) & \text { if } \quad n=1,\end{cases}
$$

and put

$$
\begin{aligned}
\mathbb{E}_{r, s, t}(\Omega):=C\left(\bar{\Omega}, \mathbb{R}_{\text {diag }}^{N \times N}\right)^{n \times n} \times L_{r}\left(\Omega, \mathbb{R}_{\text {diag }}^{N \times N}\right)^{n} & \times L_{s}\left(\Omega, \mathbb{R}^{N \times N}\right) \\
& \times L_{t}\left(\Gamma, \mathbb{R}^{N \times N}\right) \times C\left(\Gamma, \mathbb{R}_{\text {diag }}^{N \times N}\right) .
\end{aligned}
$$

Note that $\mathbb{E}^{1}(\Omega)$ and $\mathbb{E}_{r, s, t}(\Omega)$ are Banach spaces satisfying $\mathbb{E}^{1}(\Omega) \hookrightarrow \mathbb{E}_{r, s, t}(\Omega)$. 
After these preparations we can formulate the following general continuity theorem for the principle eigenvalue, whose proof is given in Section 18. Note that we allow not only all coefficients to vary but the domain as well.

Theorem 20. Let (31) be satisfied. Suppose that $\left(\Omega_{i}\right)$ is a sequence of bounded $C^{2}$ domains $C^{1}$ converging towards $\Omega$, and let $\left(\varphi_{i}\right)$ be a representation sequence for $\left(\Omega_{i}\right)$. Also suppose that $\alpha \in \mathcal{E}(\Omega)$ and $\alpha_{i} \in \mathcal{E}\left(\Omega_{i}\right)$ such that $\left(\varphi_{i}^{*} \alpha_{i}\right)$ converges in $\mathbb{E}_{r, s, t}(\Omega)$ towards $\alpha$. Then

$$
\lambda_{0}\left(\mathcal{A}\left(\alpha_{i}\right), \mathcal{B}\left(\alpha_{i}\right), \Omega_{i}\right) \rightarrow \lambda_{0}(\mathcal{A}(\alpha), \mathcal{B}(\alpha), \Omega) \text { as } i \rightarrow \infty .
$$

Furthermore, if $u$, resp. $u_{i}$, is the unique positive eigenfunction of $(\mathcal{A}(\alpha), \mathcal{B}(\alpha))$, resp. $\left(\mathcal{A}\left(\alpha_{i}\right), \mathcal{B}\left(\alpha_{i}\right)\right)$, of $W_{2}^{1}$ norm 1 then $\varphi_{i}^{*} u_{i} \rightarrow u$ in $W_{2}^{1}$.

It should be remarked that $\varphi_{i}^{*} u_{i} \rightarrow u$ in $W_{q}^{1}$, provided $r, s$, and $t$ are replaced by suitably chosen numbers $\xi, \eta$, and $\zeta$ depending on $q$ (cf. Theorem 47).

Example 21. Suppose that $\Omega$ is bounded and $f \in C^{1}\left(\bar{\Omega}, \mathbb{R}^{n}\right)$. Let $\varphi$ be the flow defined by (29) and fix $\tau^{-}<0<\tau^{+}$such that (30) is true. Let $V$ be an open neighborhood of

$$
\bigcup_{\tau^{-}<\tau<\tau^{+}} \bar{\Omega}_{\tau} \times\{\tau\}
$$

in $\mathbb{R}^{n} \times \mathbb{R}$ and suppose that $\left(\left[\bar{a}_{j k}\right],\left(\bar{a}_{1}, \ldots, \bar{a}_{n}\right), \bar{a}, \bar{b}, \bar{\chi}\right)$ belongs to

$$
\begin{aligned}
C^{1}\left(V, \mathbb{R}_{\text {diag }}^{N \times N}\right)^{n \times n} \times C\left(V, \mathbb{R}_{\text {diag }}^{N \times N}\right)^{n} & \times C\left(V, \mathbb{R}^{N \times N}\right) \\
& \times C\left(V, \mathbb{R}^{N \times N}\right) \times C\left(V, \mathbb{R}_{\text {diag }}^{N \times N}\right) .
\end{aligned}
$$

For $\tau \in\left(\tau^{-}, \tau^{+}\right)$put

$$
\alpha(\tau):=\left(\left[a_{j k}(\tau)\right],\left(a_{1}(\tau), \ldots, a_{n}(\tau)\right), a(\tau), b(\tau), \chi(\tau)\right),
$$

where

$$
a_{j k}(\tau):=\bar{a}_{j k}(\cdot, \tau)\left|\bar{\Omega}_{\tau}, \quad a_{j}(\tau):=\bar{a}_{j}(\cdot, \tau)\right| \bar{\Omega}_{\tau}, \quad a(\tau):=\bar{a}(\cdot, \tau) \mid \bar{\Omega}_{\tau}
$$

and

$$
b(\tau):=\bar{b}(\cdot, \tau)\left|\Gamma_{\tau}, \quad \chi(\tau):=\bar{\chi}(\cdot, \tau)\right| \Gamma_{\tau}
$$

with $\Gamma_{\tau}:=\partial \Omega_{\tau}=\varphi^{\tau}(\Gamma)$. Note that $\alpha(\tau) \in \mathbb{E}^{1}\left(\Omega_{\tau}\right)$.

Suppose that

$$
a(\tau) \in \mathcal{E}\left(\Omega_{\tau}\right), \quad\left(\varphi^{\tau}\right)^{*} \chi(\tau)=\chi:=\chi(0), \quad \tau^{-}<\tau<\tau^{+},
$$

and put

so that $(\mathcal{A}, \mathcal{B})=\left(\mathcal{A}_{0}, \mathcal{B}_{0}\right)$. Then

$$
\left(\mathcal{A}_{\tau}, \mathcal{B}_{\tau}\right):=(\mathcal{A}(\alpha(\tau)), \mathcal{B}(\alpha(\tau)))
$$

$$
\lambda_{0}\left(\mathcal{A}_{\tau}, \mathcal{B}_{\tau}, \Omega_{\tau}\right) \rightarrow \lambda_{0}(\mathcal{A}, \mathcal{B}, \Omega) \quad \text { as } \tau \rightarrow 0 .
$$

If $u_{\tau}$ is the unique positive eigenfunction of $\left(\mathcal{A}_{\tau}, \mathcal{B}_{\tau}\right)$ satisfying $\left\|u_{\tau}\right\|_{W_{2}^{1}\left(\Omega_{\tau}\right)}=1$ then $\left(\varphi^{\tau}\right)^{*} u_{\tau} \rightarrow u_{0}$ in $W_{2}^{1}$. 
Proof. It is easily verified that $\left(\varphi^{\tau}\right)^{*} \alpha(\tau) \rightarrow \alpha=\alpha(0)$ as $\tau \rightarrow 0$. Hence the assertion is a consequence of Theorem 20 .

Next we present a second continuity theorem for which we need some preparation.

We recall that the $C^{2}$ manifold $\bar{\Omega}$ is $C^{2}$ diffeomorphic to a $C^{\infty}$ manifold, the latter being unique up to $C^{\infty}$ diffeomorphisms (e.g., [46, Theorem II.3.4]). Thus we can assume without loss of generality that $\bar{\Omega}$ is smooth. Then $\Gamma$ is a compact oriented smooth hypersurface in $\mathbb{R}^{n}$. Hence there exists $\rho>0$ such that, setting

$$
T_{\rho}:=\left\{y+s \nu(y) \in \mathbb{R}^{n} ; y \in \Gamma,|s|<\rho\right\},
$$

the map

$$
T_{\rho} \rightarrow \Gamma \times(-1,1), \quad y+s \nu(y) \mapsto(y, s / \rho)
$$

is a smooth diffeomorphism. In other words, $T_{\rho}$ is a normal tubular neighborhood of $\Gamma$ (cf. [46, Theorem IV.5.2]). Note that $\Omega \cup T_{\rho}$ is the open $\rho$ neighborhood of $\bar{\Omega}$, that is,

$$
\Omega \cup T_{\rho}=\left\{x \in \mathbb{R}^{n} ; \operatorname{dist}(x, \bar{\Omega})<\rho\right\} .
$$

Assume that $\beta \in C^{2}(\Gamma,(-\rho, \rho))$ and put

$$
\Gamma_{\beta}:=\left\{y+\beta(y) \nu(y) \in \mathbb{R}^{n} ; y \in \Gamma\right\} .
$$

Then $\Gamma_{\beta}$ is an oriented $C^{2}$ hypersurface in $\mathbb{R}^{n}$ being contained in the tubular neighborhood $T_{\rho}$ of $\Gamma$. There exists a unique $C^{2}$ domain, $\Omega_{\beta}$, in $\mathbb{R}^{n}$ such that it is contained in $\Omega \cup T_{\rho}$ and $\Gamma_{\beta}$ is its boundary. We also define $\psi_{\beta} \in C^{2}\left(\Gamma, T_{\rho}\right)$ by

$$
\psi_{\beta}(y):=y+\beta(y) \nu(y), \quad y \in \Gamma .
$$

Suppose that

$$
\beta_{i} \in C^{2}(\Gamma,(-\rho, \rho)), \quad i \in \mathbb{N},
$$

and put $\Omega_{i}:=\Omega_{\beta_{i}}$ and $\Gamma_{i}:=\Gamma_{\beta_{i}}$. Assume that

$$
\alpha:=\left(\left[a_{j k}\right],\left(a_{1}, \ldots, a_{n}\right), a, b, \chi\right) \in \mathcal{E}(\Omega)
$$

and

$$
\alpha_{i}:=\left(\left[a_{j k,(i)}\right],\left(a_{1,(i)}, \ldots, a_{n,(i)}\right), a_{(i)}, b_{(i)}, \chi_{(i)}\right) \in \mathcal{E}\left(\Omega_{i}\right)
$$

for $i \in \mathbb{N}$, where

$$
a_{j k,(i)}=\bar{a}_{j k,(i)} \mid \bar{\Omega}
$$

for some

$$
\bar{a}_{j k,(i)} \in C^{1}\left(\Omega \cup T_{\rho}, \mathbb{R}_{\mathrm{diag}}^{N \times N}\right) .
$$

Furthermore, assume that

$$
\bar{a}_{j k,(i)} \rightarrow \bar{a}_{j k} \quad \text { in } C^{1}\left(\Omega \cup T_{\rho}, \mathbb{R}_{\text {diag }}^{N \times N}\right) \text { as } i \rightarrow \infty,
$$

where $\bar{a}_{j k} \mid \bar{\Omega}=a_{j k}$ for $1 \leq j, k \leq n$, that

$$
\beta_{i} \rightarrow 0 \text { in } C^{1}(\Gamma, \mathbb{R}) \text { and } \psi_{i}^{*} \chi_{(i)}=\chi,
$$


and that

$$
\int_{\Omega_{i} \cap \Omega}\left\{\max _{1 \leq j \leq n}\left|a_{j,(i)}-a_{j}\right|^{r}+\left|a_{(i)}-a\right|^{s}\right\} d x \rightarrow 0
$$

as well as

$$
\psi_{i}^{*} b_{(i)} \rightarrow b \quad \text { in } L_{t}\left(\Gamma, \mathbb{R}^{N \times N}\right)
$$

as $i \rightarrow \infty$.

Observe that conditions (36) and (38) are automatically satisfied if only the boundary of $\Omega$ is perturbed and $\mathcal{A}$ is kept fixed with its coefficients being defined on $\Omega \cup T_{\rho}$. Similarly, if $\bar{b} \in C^{1-}\left(\Omega \cup T_{\rho}, \mathbb{R}^{N \times N}\right)$ and $b_{(i)}:=\bar{b} \mid \Gamma_{i}$ for $i \in \mathbb{N}$ then condition (39) holds as well.

Theorem 22. Let conditions (33) - (39) be satisfied. Then

$$
\lambda_{0}\left(\mathcal{A}\left(\alpha_{i}\right), \mathcal{B}\left(\alpha_{i}\right), \Omega_{i}\right) \rightarrow \lambda_{0}(\mathcal{A}, \mathcal{B}, \Omega) \quad \text { as } i \rightarrow \infty .
$$

Furthermore, if the function $u$, resp. $u_{i}$, is the unique positive eigenfunction of $(\mathcal{A}, \mathcal{B})$, resp. $\left(\mathcal{A}\left(\alpha_{i}\right), \mathcal{B}\left(\alpha_{i}\right)\right)$ of norm 1 in $W_{2}^{1}$, resp. $W_{2}^{1}\left(\Omega_{i}, \mathbb{R}^{N}\right)$, then, given any compact subset $K$ of $\Omega$,

as $i \rightarrow \infty$.

$$
\int_{K}\left\{\left|u_{i}-u\right|^{2}+\left|\nabla u_{i}-\nabla u\right|^{2}\right\} d x \rightarrow 0
$$

This theorem, which we derive in Section 18 from Theorem 20, is - except for regularity assumptions on the Dirichlet boundary - a generalization of the continuity result in [21], where the Robin boundary and the coefficients are kept fixed and the scalar case is considered only. Of course, in the case of Dirichlet boundary conditions for a scalar equation much more general results can be obtained as has been shown by Daners [28], [29] and others (see the remarks following Theorem 18). Also see [27] for the scalar Robin problem in a $W_{2}^{1}$ setting.

\section{Minimax characterizations}

Let $\Omega$ be bounded and suppose that only the weaker hypothesis (16) is satisfied.

In this section we give further characterizations of the principal eigenvalue of $(\mathcal{A}, \mathcal{B})$. Our first theorem is related to point-wise estimates.

Theorem 23. Suppose hat either $N=1$ or $(a, b)$ is irreducible. Then

$$
\begin{array}{r}
\lambda_{0}(\mathcal{A}, \mathcal{B})=\sup \left\{\lambda \in \mathbb{R} ; \text { there exist } q \in(1, \infty) \text { and } u \in\left(W_{q}^{2}\right)^{+}\right. \\
\text {satisfying }((\mathcal{A}-\lambda) u, \mathcal{B} u)>0\} .
\end{array}
$$

Proof. Suppose that $\lambda<\lambda_{0}:=\lambda_{0}(\mathcal{A}, \mathcal{B})$. Then

$$
\lambda_{0}(\mathcal{A}-\lambda, \mathcal{B})=\lambda_{0}-\lambda>0 .
$$


Hence, given $q \in(1, \infty)$, Theorem 8 guarantees the existence of $u \in\left(W_{q}^{2}\right)^{+}$satisfying

$$
((\mathcal{A}-\lambda) u, \mathcal{B} u)=\left(\mathbf{1}_{\Omega}, 0\right) .
$$

Thus, denoting by $\lambda^{*}$ the above supremum, it follows that $\lambda_{0} \leq \lambda^{*}$.

Suppose that $\varepsilon:=\left(\lambda^{*}-\lambda_{0}\right) / 2>0$. Then there exist $q \in(1, \infty)$ and $u \in\left(W_{q}^{2}\right)^{+}$ satisfying

$$
\left(\left(\mathcal{A}-\left(\lambda^{*}-\varepsilon\right)\right) u, \mathcal{B} u\right)>0 .
$$

Thus $u$ is a positive strict supersolution for $\left(\mathcal{A}-\lambda^{*}+\varepsilon, \mathcal{B}\right)$. Hence, by Theorem 13,

$$
0<\lambda_{0}\left(\mathcal{A}-\lambda^{*}+\varepsilon, \mathcal{B}\right)=\lambda_{0}-\lambda^{*}+\varepsilon=-\varepsilon,
$$

which is impossible. This proves $\lambda_{0}=\lambda^{*}$.

Corollary 24. Suppose that $N=1$ and $n<q<\infty$. Then

$$
\lambda_{0}(\mathcal{A}, \mathcal{B})=\sup _{u \in \mathcal{P}_{\mathcal{B}}} \inf _{x \in \Omega} \frac{\mathcal{A} u(x)}{u(x)},
$$

where $\mathcal{P}_{\mathcal{B}}$ is the set of all $u \in W_{q}^{2}$ satisfying $u(x)>0$ for $x \in \Omega$ and $\mathcal{B} u \geq 0$.

Proof. It follows from the preceding theorem by observing that Theorem 15 and the above proof show that it suffices to take the supremum with respect to all strongly positive $u$.

This corollary is Theorem 4.1 in [21]. A related result can be found in [20] for the Dirichlet problem for irreducible cooperative systems.

Now we prove a minimax characterization of $\lambda_{0}(\mathcal{A}, \mathcal{B})$ which is more in the spirit of the variational characterization of eigenvalues of self-adjoint operators.

Theorem 25. Suppose that $N=1$ or $(a, b)$ is irreducible. Fix $q \in(1, \infty)$ and denote by $\mathcal{Q}$ the set of strictly positive $u \in W_{q}^{1}$ satisfying $(1-\chi) \gamma u \geq 0$. Then

$$
\lambda_{0}(\mathcal{A}, \mathcal{B})=\sup _{u \in \mathcal{Q}} \inf \frac{\mathfrak{a}(v, u)}{\langle v, u\rangle},
$$

the infimum being taken with respect to all nonzero $v \in\left(W_{q^{\prime},(1-\chi) \gamma}^{1}\right)^{+}$.

Proof. Suppose that

$$
\lambda<\lambda_{0}:=\lambda_{0}(\mathcal{A}, \mathcal{B}) \text {. }
$$

Then, as in the proof of Theorem 23, we see that

$$
\lambda_{0}(\mathcal{A}-\lambda, \mathcal{B})>0
$$

and there exists a positive strict $W_{q}^{2}$ supersolution $u$ for $(\mathcal{A}-\lambda, \mathcal{B})$. Hence $u$ is strictly positive by Theorem 15. From Green's formula we deduce that $u$ is a positive strict $W_{q}^{1}$ supersolution for $(\mathcal{A}-\lambda, \mathcal{B})$. Consequently,

$$
\mathfrak{a}(v, u) \geq \lambda\langle v, u\rangle, \quad v \in\left(W_{q^{\prime},(1-\chi) \gamma}^{1}\right)^{+},
$$


and $(1-\chi) \gamma u \geq 0$. Since $\langle v, u\rangle>0$ for each $v>0$ by the strict positivity of $u$, we see that

$$
\lambda \leq \inf _{v \in\left(W_{q^{\prime},(1-\chi) \gamma}^{1}\right)+\backslash\{0\}} \frac{\mathfrak{a}(v, u)}{\langle v, u\rangle} .
$$

This implies $\lambda_{0}:=\lambda_{0}(\mathcal{A}, \mathcal{B}) \leq \lambda^{*}$, with $\lambda^{*}$ denoting the right-hand side of (41).

Suppose that $\varepsilon:=\left(\lambda^{*}-\lambda\right) / 2>0$. Then there exists $u \in \mathcal{Q}$ satisfying

$$
\mathfrak{a}(v, u)>\left(\lambda^{*}-\varepsilon\right)\langle v, u\rangle, \quad v \in\left(W_{q^{\prime},(1-\chi) \gamma}^{1}\right)^{+} \backslash\{0\} .
$$

Hence $u$ is a positive strict $W_{q}^{1}$ supersolution for $\left(\mathcal{A}-\lambda^{*}+\varepsilon, \mathcal{B}\right)$. By invoking once more Theorem 13 we arrive at a contradiction as in the final part of the proof of Theorem 23.

\section{Concavity of the principal eigenvalue}

Suppose again that $\Omega$ is bounded and only assumption (16) is satisfied.

In this section we give an application of the minimax characterization of Theorem 25 to the study of the behavior of $\lambda_{0}(\mathcal{A}, \mathcal{B})$ as a function of $(a, b)$. For this we first note that, if $N \geq 2$, the set of all cooperative pairs $(\alpha, \beta)$ is a convex cone in the algebra

$$
\left(L_{\infty}(\Omega, \mathbb{R}) \times C^{1-}(\Gamma, \mathbb{R})\right)^{N \times N} .
$$

Given $\left(a_{j}, b_{j}\right)$ in (42) for $j=0,1$, we put

$$
\left(\mathcal{A}_{t}, \mathcal{B}_{t}\right):=(1-t)\left(\mathcal{A}_{0}, \mathcal{B}_{0}\right)+t\left(\mathcal{A}_{1}, \mathcal{B}_{1}\right), \quad 0 \leq t \leq 1,
$$

where $\left(\mathcal{A}_{j}, \mathcal{B}_{j}\right)$ are defined for $j \in\{0,1\}$ by replacing $a$ in (8) and $b$ in (9) by $a_{j}$ and $b_{j}$, respectively.

Theorem 26. Suppose that $\left(a_{0}, b_{0}\right)$ and $\left(a_{1}, b_{1}\right)$ belong to (42). Also suppose that either $N=1$ or $\left(\mathcal{A}_{t}, \mathcal{B}_{t}\right)$ is cooperative and irreducible for $0 \leq t \leq 1$. Then

$$
\lambda_{0}\left(\mathcal{A}_{t}, \mathcal{B}_{t}\right) \geq(1-t) \lambda_{0}\left(\mathcal{A}_{0}, \mathcal{B}_{0}\right)+t \lambda_{0}\left(\mathcal{A}_{1}, \mathcal{B}_{1}\right), \quad 0 \leq t \leq 1 .
$$

Proof. Let $\mathfrak{a}_{t}$ be the Dirichlet form of $\left(\mathcal{A}_{t}, \mathcal{B}_{t}\right)$. Fix $q \in(1, \infty)$ and define $\mathcal{Q}$ as in the preceding theorem. Then, given $u \in \mathcal{Q}$ and $t \in(0,1)$,

$$
\mathfrak{a}_{t}(v, u)=(1-t) \mathfrak{a}_{0}(v, u)+t \mathfrak{a}_{1}(v, u), \quad v \in\left(W_{q^{\prime},(1-\chi) \gamma}^{1}\right)^{+},
$$

implies

$$
\inf _{v} \mathfrak{a}_{t}(v, u) \geq(1-t) \inf _{v} \mathfrak{a}_{0}(v, u)+t \inf _{v} \mathfrak{a}_{1}(v, u),
$$

where the infima are taken with respect to

$$
v \in\left(W_{q^{\prime},(1-\chi) \gamma}^{1}\right)^{+} \backslash\{0\} .
$$

By passing to the suprema with respect to $u \in \mathcal{Q}$ we deduce from Theorem 25 that (43) is true. 
Remark 27. Suppose that $N>1$ and $-\left(a_{0}, b_{0}\right)$ and $-\left(a_{1}, b_{1}\right)$ are cooperative. Also suppose that either there exists a subset $\mathrm{P}$ of $\Omega$ of positive measure such that $a_{0}(x)$ and $a_{1}(x)$ are irreducible for all $x \in \mathrm{P}$, or there exists $y \in \Gamma$ with $\chi^{r}(y)=1$ for $r \in\{1, \ldots, N\}$ such that $b_{0}(y)$ and $b_{1}(y)$ are irreducible. Then $\left(\mathcal{A}_{t}, \mathcal{B}_{t}\right)$ is irreducible for $0 \leq t \leq 1$.

In the scalar case the concavity of the principal eigenvalue with respect to $a$ has been shown in [21] generalizing earlier results of López-Gómez [52] and Berestycki, Nirenberg, and Varadhan [19] for the case of Dirichlet boundary conditions (also see [18], [44], and [47]). Note that our proof does not only apply to cooperative irreducible systems but is, even in the scalar case, much simpler than the earlier ones.

As shown in the scalar case in [21] and [52] (also see the references therein), Theorem 26 is an important tool for studying eigenvalue problems with weight functions. Thanks to Theorem 26 we can now consider Steklov type eigenvalue problems also where the eigenvalue parameter can occur in the boundary condition as well, that is, problems of the type

$$
\mathcal{A} u=\lambda \alpha u \text { in } \Omega, \quad \mathcal{B} u=\lambda \beta u \text { on } \Gamma
$$

with $(\alpha, \beta)$ belonging to (42). By means of the above theorem it can be shown that the map

$$
\lambda \mapsto \lambda_{0}(\mathcal{A}-\lambda \alpha, \mathcal{B}-\lambda \beta)
$$

is convex and analytic on $\mathbb{R}$ if $N=1$, and on $\mathbb{R}^{+}$if $N>1$ and $(a, b)$ and $(\alpha, \beta)$ are such that the latter theorem can be applied. The proofs in [21], [44], or [52] carry over without changes. Since the zeros of the function (45) correspond to eigenvalues of (44) having positive eigenfunctions, we can thus extend many of the results for the case $\beta=0$ (and $N=1$ ), known so far and cited above, to (44) (also see [35], [42] for recent developments).

We remind the reader that problems of type (44) occur naturally in the study of parameter dependent nonlinear boundary value problems of the form

$$
\mathcal{A} u=\lambda f(x, u) \text { in } \Omega, \quad \mathcal{B} u=\lambda g(x, u) \text { on } \Gamma
$$

with nonlinear boundary conditions (cf. [2] for a study of such problems in the scalar case and a regular setting).

\section{Preparatory considerations}

Let $E:=E_{0}$ and $E_{1}$ be Banach spaces such that $E_{1} \stackrel{d}{\hookrightarrow} E_{0}$, and suppose that $E_{0}$ is ordered and $A \in \mathcal{H}\left(E_{1}, E_{0}\right)$. Then $A$ is said to be resolvent positive if there exists $\omega \in \mathbb{R}$ such that

$$
(\omega, \infty) \subset \rho(-A) \text { and }(\lambda+A)^{-1} \geq 0 \text { for } \lambda>\omega .
$$

We denote by $s_{+}(-A)$ the infimum of all such $\omega$.

The next two theorems are basically known and included for easy reference only.

\section{Theorem 28.}


(a) The following are equivalent:

(i) $A$ is resolvent positive.

(ii) $(\lambda+A)^{-1} \geq 0$ for $\lambda>s(-A)$, that is, $s_{+}(-A) \leq s(-A)$.

(iii) The semigroup $\mathcal{U}_{A}$ is positive.

(b) If $A$ is resolvent positive and $\sigma(A) \neq \varnothing$ then $-s_{+}(-A) \in \sigma(A)$.

Proof. (a) It is known (e.g., [8, Remark II.5.1.2] or [25, Proposition 9.2]) that $s(-A)$ equals the type of $-A$, the latter being the infimum of all $\omega \in \mathbb{R}$ such that there exists $M \geq 1$ satisfying

$$
\left\|U_{A}(t)\right\|_{\mathcal{L}\left(E_{0}\right)} \leq M e^{\omega t}
$$

for $t \geq 0$. Hence the assertion follows from well-known results in semigroup theory (eg., [25, Proposition 7.1]).

(b) This follows from part (b) of the proof of Proposition 3.11.2 in [15] (by observing that it is valid without the standing hypotheses of those authors that the positive cone is normal and generating ).

The positive cone $E^{+}$is said to be generating if $E=E^{+}-E^{+}$. It is normal if each order interval

$$
[x, y]:=\{z \in E ; x \leq z \leq y\}
$$

is bounded. Note, in particular, that $E^{+}$is normal and generating if $E$ is a Banach lattice.

Theorem 29. Suppose that $E^{+}$is normal and generating. Then:

(i) $s_{+}(-A)=s(-A)$;

(ii) If $\lambda \in \rho(-A) \cap \mathbb{R}$ and $(\lambda+A)^{-1} \geq 0$ then $\lambda>s(-A)$.

Proof. See [15, Proposition 3.11.2] or [25, Theorem 7.4].

The operator $A$ is said to be inverse positive if $u \in E_{1}$ and $A u \geq 0$ imply $u \geq 0$.

Corollary 30. Let $E$ be a Banach lattice and suppose that $A$ is resolvent positive. If $\lambda_{0}(A)>0$ then $A$ is inverse positive. Conversely, if $A$ is inverse positive and $A$ is surjective then $\lambda_{0}(A)>0$.

Proof. If $A$ is inverse positive then it is injective. Thus $0 \in \rho(A)$ if, in addition, $A$ is surjective. Now the assertion is obvious.

Let $E$ be a Banach lattice. Then $x \in E^{+}$is said to be a quasi-interior point of $E^{+}$iff $\langle\varphi, x\rangle>0$ for every $\varphi \in\left(E^{\prime}\right)^{+} \backslash\{0\}$. Clearly, $u$ is a quasi-interior point of $L_{q}^{+}$iff $u$ is strictly positive. 
A positive linear operator $K \in \mathcal{L}(E)$ is called irreducible iff there exists $\mu>r(K)$, with $r(K)$ being the spectral radius of $K$, such that $K(\mu-K)^{-1} x$ is for each $x \in E^{+} \backslash\{0\}$ a quasi-interior point of $E^{+}$. It is strongly irreducible iff $K x$ is for each $x \in E^{+} \backslash\{0\}$ a quasi-interior point of $E^{+}$. Clearly, every strongly irreducible operator is irreducible.

The semigroup $\mathcal{U}_{A}$ is said to be strongly irreducible iff $U_{A}(t)$ has this property for each $t>0$.

Theorem 31. Suppose that $E$ is a Banach lattice and $A$ is resolvent positive. Then the following are equivalent:

(i) There exists $\lambda>-\lambda_{0}$ such that $(\lambda+A)^{-1}$ is irreducible.

(ii) $(\lambda+A)^{-1}$ is for each $\lambda>-\lambda_{0}$ strongly irreducible.

(iii) $\mathcal{U}_{A}$ is strongly irreducible.

Proof. It follows from [65, App. 3.1] that the preceding definition of a positive irreducible bounded linear operator is equivalent to the one used in [25, Section 7.1]. Hence the assertion is implied by [25, Proposition 7.6 and Corollary 7.8].

Suppose that

$$
E_{0} \stackrel{d}{\hookrightarrow} E_{-1}, \quad A_{-1} \in \mathcal{H}\left(E_{0}, E_{-1}\right), \quad A_{-1} \supset A_{0}:=A .
$$

Then we denote for $j \in\{-1,0\}$ by $N_{j}(\lambda)$ the algebraic eigenspace of the eigenvalue $\lambda$ of $A_{j}$.

Lemma 32. Let (46) be satisfied. Then

$$
\sigma_{p}\left(A_{-1}\right)=\sigma_{p}\left(A_{0}\right)
$$

Furthermore,

$$
N_{-1}(\lambda)=N_{0}(\lambda), \quad \lambda \in \sigma_{p}\left(A_{0}\right)
$$

Proof. (i) It is clear that $\sigma_{p}\left(A_{0}\right) \subset \sigma_{p}\left(A_{-1}\right)$ and $N_{0}(\lambda) \subset N_{-1}(\lambda)$ for $\lambda \in \sigma_{p}\left(A_{0}\right)$.

(ii) Suppose that there exist $\lambda \in \mathbb{C}$ and $x, y \in E_{0}$ satisfying

$$
A_{-1} x=\lambda x-y \text {. }
$$

Fix $\omega \in \rho\left(-A_{0}\right) \cap \rho\left(-A_{-1}\right)$. Then (47) is equivalent to

$$
x=\left(\omega+A_{-1}\right)^{-1}((\omega+\lambda) x-y) .
$$

From $A_{-1} \supset A_{0}$ it follows that

$$
\left(\omega+A_{-1}\right)^{-1} \supset\left(\omega+A_{0}\right)^{-1} .
$$

Thus we deduce from $(\omega+\lambda) x-y \in E_{0}$ and (48), thanks to $\left(\omega+A_{0}\right)^{-1}\left(E_{0}\right) \subset E_{1}$, that $x \in E_{1}$ and, consequently, $A_{0} x=\lambda x-y$. 
(iii) Suppose that $\lambda \in \mathbb{C}, m \in \mathbb{N}$, and

$$
x \in \operatorname{ker}\left[\left(\lambda-A_{-1}\right)^{m+1}\right] .
$$

Then there exist $x_{0}, \ldots, x_{m} \in E_{0}$ satisfying $x_{0}=x$ and

$$
A_{-1} x_{k}=\lambda x_{k}-x_{k+1}, \quad 0 \leq k \leq m,
$$

where $x_{m+1}:=0$. Thus we deduce from (ii) by backwards induction that $x_{k} \in E_{1}$ for $0 \leq k \leq m$ and that

$$
x_{0} \in \operatorname{ker}\left[\left(\lambda-A_{0}\right)^{m+1}\right] .
$$

This implies $N_{-1}(\lambda) \subset N_{0}(\lambda)$.

Next we prove a perturbation theorem for resolvent positive generators of analytic semigroups.

Proposition 33. Suppose that $\theta \in(0,1)$ and $(\cdot, \cdot)_{\theta}$ is an interpolation functor of exponent $\theta$. Put $E_{\theta}:=\left(E_{0}, E_{1}\right)_{\theta}$. If $B \in \mathcal{L}\left(E_{\theta}, E_{0}\right)$ then

$$
A-B \in \mathcal{H}\left(E_{1}, E_{0}\right) \text {. }
$$

If $A$ is resolvent positive and $B \geq 0$ then $A-B$ is also resolvent positive.

Proof. Since $A \in \mathcal{H}\left(E_{1}, E_{0}\right)$ there exist positive constants $M$ and $\omega$ such that

$$
|\lambda|^{1-j}\left\|(\lambda+A)^{-1}\right\|_{\mathcal{L}\left(E_{0}, E_{j}\right)} \leq M, \quad \operatorname{Re} \lambda>\omega, \quad j=0,1 .
$$

Thus, by interpolation, there exists $M_{1}$ such that

$$
\left\|(\lambda+A)^{-1}\right\|_{\mathcal{L}\left(E_{0}, E_{\theta}\right)} \leq M_{1} /|\lambda|^{1-\theta}, \quad \operatorname{Re} \lambda>\omega .
$$

Hence we can find $\omega_{1} \geq \omega$ such that

$$
\left\|B(\lambda+A)^{-1}\right\|_{\mathcal{L}\left(E_{0}\right)} \leq 1 / 2, \quad \operatorname{Re} \lambda>\omega_{1} .
$$

It follows that $1-B(\lambda+A)^{-1} \in \mathcal{L}\left(E_{0}\right)$ has an inverse on $E_{0}$, bounded by 2 , and

$$
\left(1-B(\lambda+A)^{-1}\right)^{-1}=\sum_{j=0}^{\infty}\left[B(\lambda+A)^{-1}\right]^{j}, \quad \operatorname{Re} \lambda>\omega_{1},
$$

in $\mathcal{L}\left(E_{0}\right)$. Hence

$$
\left[\operatorname{Re} \lambda>\omega_{1}\right] \subset \rho(-A+B)
$$

and

$$
(\lambda+A-B)^{-1}=(\lambda+A)^{-1}\left(1-B(\lambda+A)^{-1}\right)^{-1}, \quad \operatorname{Re} \lambda>\omega_{1},
$$

so that

$$
\left\|(\lambda+A-B)^{-1}\right\|_{\mathcal{L}\left(E_{0}, E_{j}\right)} \leq 2 M_{1} /|\lambda|^{1-j}, \quad \operatorname{Re} \lambda>\omega_{1}, \quad j=0,1 .
$$

This proves that $A-B \in \mathcal{H}\left(E_{1}, E_{0}\right)$. Furthermore, if $B \geq 0$ and $A$ is resolvent positive, we deduce from (49) and (50) that $(\lambda+A-B)^{-1} \geq 0$ for $\lambda>\omega_{1}$. 
It should be remarked that the first part of the assertion is well-known (eg., (I.2.2.2) and Theorem I.1.3.1 in [8]).

The next result shows that the set of resolvent positive operators in $\mathcal{H}\left(E_{1}, E_{0}\right)$ is closed.

Proposition 34. Let $\left(A_{j}\right)$ be a sequence in $\mathcal{H}\left(E_{1}, E_{0}\right)$ converging in $\mathcal{L}\left(E_{1}, E_{0}\right)$ towards $A$. If each $A_{j}$ is resolvent positive then $A$ is resolvent positive as well.

Proof. It follows from [8, Corollary I.1.3.2] that there exist $\kappa \geq 1$ and $\omega>0$ such that $[\operatorname{Re} z>\omega]$ belongs to $\rho(-A) \cap \rho\left(-A_{j}\right)$ and

$$
\left\|(\lambda+A)^{-1}\right\|_{\mathcal{L}\left(E_{0}, E_{1}\right)}+|\lambda|\left\|\left(\lambda+A_{j}\right)^{-1}\right\|_{\mathcal{L}\left(E_{0}\right)} \leq \kappa, \quad \operatorname{Re} \lambda>\omega, \quad j \in \mathbb{N} .
$$

Hence we infer from

$$
\left(\lambda+A_{j}\right)^{-1}-(\lambda+A)^{-1}=\left(\lambda+A_{j}\right)^{-1}\left(A-A_{j}\right)(\lambda+A)^{-1}, \quad \operatorname{Re} \lambda>\omega,
$$

that

$$
\left\|\left(\lambda+A_{j}\right)^{-1}-(\lambda+A)^{-1}\right\|_{\mathcal{L}\left(E_{0}\right)} \leq|\lambda|^{-1} \kappa^{2}\left\|A-A_{j}\right\|_{\mathcal{L}\left(E_{1}, E_{0}\right)}, \quad \operatorname{Re} \lambda>\omega
$$

Thus, in particular,

$$
\left(\lambda+A_{j}\right)^{-1} x \rightarrow(\lambda+A)^{-1} x
$$

in $E_{0}$ for $\lambda>\omega$ and $x \in E_{0}$. Now the assertion is a consequence of the closedness of the positive cone.

\section{The strong maximum principle for the scalar case}

In this section we suppose that $N=1$ and set $\Gamma_{j}:=\Gamma_{j}^{1}$ for $j=0,1$, of course. Then we put

$$
\mathcal{A}_{0} u:=-a_{j k} \partial_{j} \partial_{k} u+a_{j} \partial_{j} u+a u,
$$

where $a_{j k}=a_{k j}, a_{j}, a \in L_{\infty}$, with $\left[a_{j k}(x)\right] \in \mathbb{R}^{n \times n}$ being positive definite for a.a. $x \in \Omega$. We also put

$$
\mathcal{B}_{0} u:= \begin{cases}u & \text { on } \Gamma_{0}, \\ \partial_{\beta} u+b u & \text { on } \Gamma_{1},\end{cases}
$$

where $\beta$ is an outward pointing nowhere tangent $C^{1}$ vector field on $\Gamma_{1}$, and $b$ is a $C^{1-}$ function on $\Gamma_{1}$. Clearly, in this case the derivative $\partial_{\beta}$ is used in the definition of strong positivity.

The following theorem is the basis for the proofs of the following sections. It slightly improves [3, Theorem 6.1]. Its importance stems from the fact that there is no sign restriction for $b$.

Theorem 35. There exists $\omega_{0} \in \mathbb{R}$ such that $\left(\mathcal{A}_{0}+\omega, \mathcal{B}_{0}\right)$ satisfies for $\omega>\omega_{0}$ the strong maximum principle. 
Proof. If $\Omega$ is bounded, this is a reformulation of Theorem 6.1 in [3] (where the statement is incomplete since the condition $u(y)=0$ for the validity of $\partial_{\beta} u(y)<0$ is missing). Since

$$
W_{q}^{2} \hookrightarrow C_{0}^{1}(\bar{\Omega}), \quad q>n,
$$

the same proof applies if it is only supposed that $\Gamma$ is compact, provided Lemma 5.1 in [3] is valid. But this follows easily from the proof of the much more general Theorem B.3 in [5].

\section{Remarks 36.}

(a) The proof of [3, Lemma 5.1] is somewhat complicated and perhaps not too transparent. By restricting the arguments leading to Theorem B.3 in [5] to the relevant cases $k=0$ and $k=1$, one gets a simpler and more lucid demonstration.

(b) Suppose that $(a, b) \geq 0$. Then $\omega_{0} \leq 0$. Furthermore, $\left(\mathcal{A}_{0}, \mathcal{B}_{0}\right)$ satisfies the strong maximum principle unless $\Omega$ is bounded, $\Gamma=\Gamma_{1}$, and $(a, b)=(0,0)$.

Proof. This is a consequence of the classical maximum principle.

\section{Strong and weak solutions}

We return to the case of a general $N \in \mathbb{N}^{\times}$and the hypotheses of Section 2 . We set $W_{q, \mathcal{B}}^{0}:=L_{q}$ and define linear operators

$$
A_{k-2} \in \mathcal{L}\left(W_{q, \mathcal{B}}^{k}, W_{q, \mathcal{B}}^{k-2}\right), \quad k \in\{0,1\}
$$

by

$$
\left\langle v, A_{-1} u\right\rangle:=\mathfrak{a}(v, u), \quad(v, u) \in W_{q^{\prime},(1-\chi) \gamma}^{1} \times W_{q,(1-\chi) \gamma}^{1}
$$

and

$$
\left\langle v, A_{-2} u\right\rangle:=\left\langle\mathcal{A}^{\sharp} v, u\right\rangle, \quad(v, u) \in W_{q^{\prime}, \mathcal{B}^{\sharp}}^{2} \times L_{q},
$$

respectively. $A_{-k}$ is called $W_{q, \mathcal{B}}^{-k}$ realization of $(\mathcal{A}, \mathcal{B})$. We also put $A_{0}:=A$ and denote by $N_{-j}(\lambda)$ the algebraic eigenspace of $\lambda \in \sigma_{p}\left(A_{-j}\right)$ for $j \in\{0,1,2\}$.

Theorem 37. For $j \in\{1,2\}$

(i) $A_{j-2} \in \mathcal{H}\left(W_{q, \mathcal{B}}^{j}, W_{q, \mathcal{B}}^{j-2}\right)$;

(ii) $A_{-2} \supset A_{-1} \supset A_{0}$;

(iii) $\sigma\left(A_{-j}\right)=\sigma\left(A_{0}\right)$ and $\sigma_{p}\left(A_{-j}\right)=\sigma_{p}\left(A_{0}\right)$. If $\lambda \in \sigma_{p}\left(A_{0}\right)$ then $N_{-j}(\lambda)=N_{0}(\lambda)$.

Proof. Fix $\omega>s\left(-A_{0}\right)$ and let $\left[\left(E_{\alpha}, B_{\alpha}\right) ; \alpha \in \mathbb{R}\right]$ be the interpolation extrapolation scale generated by $\left(E_{0}, B_{0}\right):=\left(L_{q}, \omega+A_{0}\right)$ and the complex interpolation functors $[\cdot, \cdot]_{\theta}$, $0<\theta<1$. (We refer to [8, Chapter V] for the general interpolation extrapolation theory, 
and to [7, Section 6] for a summary of the main results.) Then (cf. Theorems 7.1 and 8.3 in [7] and observe that they remain valid if it is only assumed that $\Gamma$ is compact) we find that

$$
E_{-j / 2} \doteq W_{q, \mathcal{B}}^{-j}, \quad B_{-j / 2}=A_{-j},
$$

where $\doteq$ means 'equal except for equivalent norms'. Hence (i), (ii), and the equality of $\sigma\left(A_{-j}\right)$ and $\sigma\left(A_{0}\right)$ follow from (15) and the general interpolation extrapolation theory (cf. Theorems V.1.4.6 and V.2.1.3 as well as Corollary V.2.1.4 in [8]). The remaining part of (iii) is now a consequence of (i), (ii), and Lemma 32.

Remark 38. Suppose that only the weaker assumption (16) is satisfied. Then Theorem 37 remains valid for $j=1$ and with $A_{-2}$ being omitted in (ii).

Proof. Of course, the interpolation extrapolation scale generated by $\left(L_{q}, \omega+A_{0}\right)$ is still well-defined. However, since in this case the dual of $A$ is not explicitly known, the space $E_{-1}$ cannot be identified in terms of a known space of distributions. But it is not difficult to see that

is still true.

$$
E_{-1 / 2} \doteq W_{q, \mathcal{B}}^{-1}
$$

The next theorem concerns the solvability of the nonhomogeneous problem (20) and the parameter dependent boundary value problem

$$
(\lambda+\mathcal{A}) u=f \text { in } \Omega, \quad \mathcal{B} u=g \text { on } \Gamma .
$$

\section{Theorem 39.}

(i) Every strong $W_{q}^{2}$ solution of (20) is a weak $W_{q}^{1}$ solution, and each weak $W_{q}^{1}$ solution is a very weak $L_{q}$ solution.

(ii) Suppose that $\lambda \in \rho(-A), j \in\{0,1,2\}$, and $(f, g) \in W_{q, \mathcal{B}}^{j-2} \times \partial W_{q}^{j}$. Then (53) has a unique $W_{q}^{j}$ solution.

(iii) If $(f, g) \in W_{q, \mathcal{B}}^{-1} \times \partial W_{q}^{1}$ then every $L_{q}$ solution of (20) is a $W_{q}^{1}$ solution. Similarly, if $(f, g) \in L_{q} \times \partial W_{q}^{2}$ then every $W_{q}^{1}$ solution is a $W_{q}^{2}$ solution.

Proof. (i) is an easy consequence of Green's formulas.

(ii) First suppose that $j=2$. From [5, Theorem B.3] we know that there exists

$$
\mathcal{R}_{2} \in \mathcal{L}\left(\partial W_{q}^{2}, W_{q}^{2}\right)
$$

satisfying $\mathcal{B R}_{2} \varphi=\varphi$ for $\varphi \in \partial W_{q}^{2}$. Set $w:=\mathcal{R}_{2} g$. Then $u$ is a $W_{q}^{2}$ solution of (53) iff $v:=u-w$ satisfies

$$
(\lambda+\mathcal{A}) v=h \text { in } \Omega, \quad \mathcal{B} v=0 \text { on } \Gamma
$$

where

$$
h:=f-(\lambda+\mathcal{A}) w \in L_{q},
$$

that is, iff $(\lambda+A) v=h$ in $L_{q}$. This proves the assertion if $j=2$. 
Suppose that $j=1$. The trace operator is a continuous retraction from the space $W_{q}^{1}$ onto $W_{q}^{1-1 / q}(\Gamma)$, that is, there exists

$$
\mathcal{R} \in \mathcal{L}\left(W_{q}^{1-1 / q}(\Gamma), W_{q}^{1}\right)
$$

with $\gamma \mathcal{R} \varphi=\varphi$ for $\varphi \in W_{q}^{1-1 / q}(\Gamma)$ (e.g., [5, (B.21)-(B.23)]). Set $w:=\mathcal{R}(1-\chi) g$. Then $u$ is a $W_{q}^{1}$ solution of (53) iff $v:=u-w \in W_{q}^{1}$ satisfies

$$
\lambda\langle\varphi, v\rangle+\mathfrak{a}(\varphi, v)=\langle\varphi, f\rangle-\lambda\langle\varphi, w\rangle-\mathfrak{a}(\varphi, w), \quad \varphi \in W_{q^{\prime},(1-\chi) \gamma}^{1}
$$

and

$$
(1-\chi) \gamma v=0
$$

thanks to $\chi^{2}=\chi$. Since $\mathfrak{a}$ is a continuous bilinear form on $W_{q^{\prime},(1-\chi) \gamma}^{1} \times W_{q}^{1}$, the right-hand side of (54) defines an element $h$ in $W_{q, \mathcal{B}}^{-1}$. Thus (54) and (55) are equivalent to

$$
v \in W_{q,(1-\chi) \gamma}^{1}, \quad\left(\lambda+A_{-1}\right) v=h .
$$

Now the assertion for $j=1$ follows from $\sigma\left(A_{-1}\right)=\sigma(A)$.

Finally, assume that $j=0$. Note that

$$
(1-\chi) \partial_{\mu} \in \mathcal{L}\left(W_{q^{\prime}, \mathcal{B}^{\sharp}}^{2}, W_{q^{\prime}}^{1-1 / q^{\prime}}\left(\Gamma_{0}\right)\right) .
$$

Hence

Similarly,

$$
\left(\partial_{\mu}\right)^{\prime}(1-\chi) \in \mathcal{L}\left(W_{q}^{-1 / q}\left(\Gamma_{0}\right), W_{q, \mathcal{B}}^{-2}\right)
$$

and, consequently,

$$
\chi \gamma \in \mathcal{L}\left(W_{q^{\prime}, \mathcal{B}^{\sharp}}^{2}, W_{q^{\prime}}^{2-1 / q^{\prime}}\left(\Gamma_{1}\right)\right)
$$

$$
\gamma^{\prime} \chi \in \mathcal{L}\left(W_{q}^{-1-1 / q}\left(\Gamma_{1}\right), W_{q, \mathcal{B}}^{-2}\right)
$$

From this and (22) we infer that $u$ is an $L_{q}$ solution of (53) iff

$$
\left(\lambda+A_{-2}\right) u=h,
$$

where

$$
h:=f+\left(\partial_{\mu}\right)^{\prime}(\chi-1) g+\gamma^{\prime} \chi g \in W_{q, \mathcal{B}}^{-2} .
$$

Thus $\sigma\left(A_{-2}\right)=\sigma(A)$ implies the assertion in this case also.

(iii) Suppose that $(f, g) \in W_{q, \mathcal{B}}^{-1} \times \partial W_{q}^{1}$ and $u$ is an $L_{q}$ solution of (20). Then there exists $\omega>0$ such that

$$
\lambda_{\omega}:=\omega+\lambda \in \rho(-A) .
$$

Hence (20) is equivalent to

$$
\left(\lambda_{\omega}+\mathcal{A}\right) u=f_{\omega} \text { in } \Omega, \quad \mathcal{B} u=g \text { on } \Gamma
$$

where

$$
f_{\omega}:=f+\omega u \in W_{q, \mathcal{B}}^{-1}
$$

Thus (ii) implies that (56) has a unique $W_{q}^{1}$ solution $v$. From (i) we infer that $v$ is an $L_{q}$ solution of (56). Since it is unique, by (ii), it follows that $u=v$, that is, $u \in W_{q, \mathcal{B}}^{1}$. This proves the first assertion. The second one follows by similar arguments. 
Remark 40. If we presuppose only condition (16) then Theorem 39 is valid with any reference to $L_{q}$ solutions being omitted.

Proof. This follows from the above proof and Remark 38.

\section{Resolvent positivity}

The next theorem is the basis of all the following positivity results.

\section{Theorem 41.}

(i) $W_{q, \mathcal{B}}^{-1}$ and $W_{q, \mathcal{B}}^{-2}$ are $O B S s$ and the natural injection maps (19) are positive.

(ii) $\left(W_{q, \mathcal{B}}^{j}\right)^{+}$is dense in $\left(W_{q, \mathcal{B}}^{k}\right)^{+}$for $-2 \leq k<j \leq 2$.

(iii) $A_{-j}$ are resolvent positive for $j \in\{0,1,2\}$.

Proof. (1) First we assume that

$$
a \in L_{\infty}\left(\Omega, \mathbb{R}_{\mathrm{diag}}^{N \times N}\right) \quad \text { and } \quad b \in C^{1-}\left(\Gamma, \mathbb{R}_{\mathrm{diag}}^{N \times N}\right) .
$$

Then Theorem 35 applies to the boundary value problem $\left(\mathcal{A}^{r}, \mathcal{B}^{r}\right)$ for $1 \leq r \leq N$. Hence there exists $\omega_{0}>0$ such that

$$
\left(\lambda+A_{0}\right)^{-1} v \geq 0, \quad \lambda>\omega_{0}, \quad v \in \mathcal{D}^{+},
$$

where $\mathcal{D}:=\mathcal{D}\left(\Omega, \mathbb{R}^{N}\right)$ is the space of all smooth $\mathbb{R}^{N}$ valued functions with compact support in $\Omega$. Since $\left(\lambda+A_{0}\right)^{-1} \in \mathcal{L}\left(L_{q}\right)$ and $\mathcal{D}^{+}$is dense in $L_{q}^{+}$it follows that (57) is true for all $v \in L_{q}^{+}$. Thus $A_{0}$ is resolvent positive.

The same arguments show that $A^{\sharp}$, the $L_{q^{\prime}}$ realization of $\left(\mathcal{A}^{\sharp}, \mathcal{B}^{\sharp}\right)$, is also resolvent positive. Note that $L_{q^{\prime}}^{+}$is generating since $L_{q^{\prime}}$ is a Banach lattice. Thus, fixing $\lambda>s\left(-A^{\sharp}\right)$ and setting

$$
P:=\left(\lambda+A^{\sharp}\right)^{-1} L_{q^{\prime}}^{+},
$$

it follows from Theorem 28 that

$$
P \subset\left(W_{q^{\prime}, \mathcal{B}^{\sharp}}^{2}\right)^{+} \quad \text { and } \quad W_{q^{\prime}, \mathcal{B}^{\sharp}}^{2}=P-P \text {. }
$$

Hence $\left(W_{q^{\prime}, \mathcal{B}^{\sharp}}^{2}\right)^{+}$is generating, thus total. Now (cf. the proof of Theorem 37) Theorems V.1.5.12, V.2.3.2, V.2.7.2, and Corollary V.2.7.3 in [8] imply that (i)-(iii) are true in this case.

(2) We consider the general case. First we observe that $W_{q, \mathcal{B}}^{1}$ and $W_{q, \mathcal{B}}^{-1}$ are independent of $a$ and $b$. Hence it follows from step (1) that they are $O B S s$, the injection maps

$$
W_{q, \mathcal{B}}^{1} \hookrightarrow L_{q} \hookrightarrow W_{q, \mathcal{B}}^{-1}
$$

are positive, $\left(W_{q, \mathcal{B}}^{1}\right)^{+}$is dense in $L_{q}^{+}$, and the latter cone is dense in $\left(W_{q, \mathcal{B}}^{-1}\right)^{+}$. 
Recall (25) and (26). Define $\left(\mathcal{A}^{\triangle}, \mathcal{B}^{\triangle}\right)$ by replacing $a$ and $b$ in the definition of $(\mathcal{A}, \mathcal{B})$ by $a^{\triangle}$ and $b^{\triangle}$, respectively. Then step (1) implies that $A_{-1}^{\triangle}$, the $W_{q, \mathcal{B}}^{-1}$ realization of $\left(\mathcal{A}^{\triangle}, \mathcal{B}^{\triangle}\right)$, is well-defined, belongs to $\mathcal{H}\left(W_{q, \mathcal{B}}^{1}, W_{q, \mathcal{B}}^{-1}\right)$, and is resolvent positive.

Fix $s \in(1 / q, 1)$ and put

$$
W_{q, \mathcal{B}}^{s}:=W_{q,(1-\chi) \gamma}^{s}:=\left\{v \in W_{q}^{s} ;(1-\chi) \gamma v=0\right\} .
$$

Then [7, Theorem 7.2] implies that

$$
W_{q, \mathcal{B}}^{s} \doteq\left(W_{q, \mathcal{B}}^{-1}, W_{q, \mathcal{B}}^{1}\right)_{(1+s) / 2, q}
$$

where $(\cdot, \cdot)_{\theta, p}$ are the real interpolation functors for $0<\theta<1$ and $1 \leq p \leq \infty$.

By the trace theorem,

$$
\gamma_{s}:=\gamma \mid W_{q, \mathcal{B}}^{s} \in \mathcal{L}\left(W_{q, \mathcal{B}}^{s}, L_{q}\left(\Gamma_{1}\right)\right)
$$

and

where

$$
\gamma_{1}^{\prime} \in \mathcal{L}\left(L_{q}\left(\Gamma_{1}\right), W_{q, \mathcal{B}}^{-1}\right)
$$

Consequently, setting

$$
\gamma_{1}:=\gamma \mid W_{q^{\prime},(1-\chi) \gamma}^{1} \in \mathcal{L}\left(W_{q^{\prime},(1-\chi) \gamma}^{1}, L_{q^{\prime}}\left(\Gamma_{1}\right)\right) .
$$

$$
B u:=a^{\bullet} u+\gamma_{1}^{\prime} \chi b^{\bullet} \gamma_{s} u, \quad u \in W_{q, \mathcal{B}}^{s},
$$

it follows from $\left(a^{\bullet}, b^{\bullet}\right) \in L_{\infty}\left(\Omega, \mathbb{R}^{N \times N}\right) \times L_{\infty}\left(\Gamma, \mathbb{R}^{N \times N}\right)$,

$$
W_{q, \mathcal{B}}^{s} \hookrightarrow L_{q} \hookrightarrow W_{q, \mathcal{B}}^{-1}
$$

and (58) and (59) that $B \in \mathcal{L}\left(W_{q, \mathcal{B}}^{s}, W_{q, \mathcal{B}}^{-1}\right)$. Furthermore, $B \geq 0$ thanks to the positivity of $\left(a^{\bullet}, b^{\bullet}\right)$, of the trace operators $\gamma_{s}$ and $\gamma_{1}$, and of the injection maps (60), and thanks to the fact that $L_{q}^{+}$and $\left(W_{q, \mathcal{B}}^{-1}\right)^{+}$are the dual cones of the positive cones of $L_{q^{\prime}}$ and $W_{q^{\prime},(1-\chi) \gamma}^{1}$, respectively. Note that

$$
\left\langle v,\left(A_{-1}^{\triangle}-B\right) u\right\rangle=\mathfrak{a}(v, u), \quad(v, u) \in W_{q^{\prime},(1-\chi) \gamma}^{1} \times W_{q,(1-\chi) \gamma}^{1},
$$

so that

$$
A_{-1}=A_{-1}^{\triangle}-B
$$

Hence Proposition 33 guarantees that $A_{-1}$ is resolvent positive.

From Theorem 37 we infer that

$$
\left(\lambda+A_{-2}\right)^{-1} \supset\left(\lambda+A_{-1}\right)^{-1} \supset\left(\lambda+A_{0}\right)^{-1}, \quad \lambda>s\left(-A_{0}\right) .
$$

Thus $A_{0}$ is resolvent positive as well. The same arguments apply to the boundary value problem $\left(\mathcal{A}^{\sharp}, \mathcal{B}^{\sharp}\right)$ and guarantee that $A_{0}^{\sharp}$ is resolvent positive. Thus we see, as in step (1), that $\left(W_{q, \mathcal{B}}^{-2}\right)^{+}$is a proper cone, that is, $W_{q, \mathcal{B}}^{-2}$ is an $O B S$. Now the remaining assertions follow by the arguments of step (1).

Remark 42. Suppose that only the weaker assumption (16) is satisfied. Then Theorem 41 remains true if all assertions involving $W_{q, \mathcal{B}}^{-2}$ and $A_{-2}$ are omitted. 
Proof. This follows from Remark 38.

\section{Proofs of the weak maximum principles}

Now it is not difficult to prove the theorems presented in Sections 4 and 5 .

Proof of Theorem 6 (a) Theorem 41(iii) guarantees that $A$ is resolvent positive. Thus (3) and (4) follow from Theorems 28 and 29, since $L_{q}$ is a Banach lattice.

(b) If $(\mathcal{A}, \mathcal{B})$ is inverse positive on $W_{q}^{2}$ then $A$ is inverse positive. Thus, if $A$ is surjective then $\lambda_{0}>0$ by Corollary 30 .

From (52) we infer that $(\mathcal{A}, \mathcal{B})$ satisfies the very weak maximum principle iff $A_{-2}$ is inverse positive. Suppose that $\lambda_{0}>0$. Then $A$ is inverse positive by Corollary 30. Since $\lambda_{0}=\lambda_{0}\left(-A_{-2}\right)$ by Theorem 37(iii), it follows that

$$
0 \in \rho\left(A_{-2}\right) \text {. }
$$

Also suppose that $u \in L_{q}$ and $A_{-2} u \geq 0$. Then Theorem 41(ii) guarantees the existence of a sequence $\left(f_{k}\right)$ in $L_{q}^{+}$converging in $W_{q, \mathcal{B}}^{-2}$ towards $f:=A_{-2} u$. Hence the sequence $\left(u_{k}\right)$, where $u_{k}:=\left(A_{-2}\right)^{-1} f_{k}$, converges in $L_{q}$ towards $u=\left(A_{-2}\right)^{-1} f$. Since $A_{-2} \supset A$ by Theorem 37(ii), it follows that $\left(A_{-2}\right)^{-1} \supset A^{-1}$. Thus $u_{k} \in W_{q, \mathcal{B}}^{2}$ and

$$
A u_{k}=f_{k} \geq 0 \text {. }
$$

Consequently, $u_{k} \geq 0$ by the inverse positivity of $A$. Hence Theorem 41(i) implies $u \geq 0$. This shows that $A_{-2}$ is inverse positive and proves (2).

(c) Suppose that $u \in W_{q}^{1}$ satisfies assumption (18). Then we define $f \in\left(W_{q, \mathcal{B}}^{-1}\right)^{+}$by

We also set

$$
\langle v, f\rangle:=\mathfrak{a}(v, u), \quad v \in W_{q^{\prime},(1-\chi) \gamma}^{1} .
$$

By Greens's formula,

$$
g:=(1-\chi) \gamma u \in\left(W_{q}^{1-1 / q}\left(\Gamma_{0}\right)\right)^{+} \hookrightarrow\left(\partial W_{q}^{0}\right)^{+} .
$$

$$
\begin{aligned}
\left\langle\mathcal{A}^{\sharp} v, u\right\rangle & =\mathfrak{a}(v, u)+\left\langle(\chi-1) \partial_{\mu} v, \gamma u\right\rangle_{\Gamma} \\
& =\langle v, f\rangle+\left\langle\partial_{\mu} v,(\chi-1) g\right\rangle_{\Gamma} \geq 0
\end{aligned}
$$

for $v \in\left(W_{q^{\prime}, \mathcal{B}^{\sharp}}^{2}\right)^{+}$. Thus $u \geq 0$ if (i) is true. Hence (i) implies (ii).

Suppose that $u \in W_{q}^{2}$ satisfies $(\mathcal{A} u, \mathcal{B} u) \geq 0$. Then

$$
(f, g):=(\mathcal{A} u, \mathcal{B} u) \in\left(L_{q} \times \partial W_{q}^{2}\right)^{+} \hookrightarrow\left(W_{q, \mathcal{B}}^{-1} \times \partial W_{q}^{1}\right)^{+}
$$

by (13). From Green's formula we infer that

$$
\mathfrak{a}(v, u)=\langle v, \mathcal{A} u\rangle+\langle\chi \gamma v, \mathcal{B} u\rangle_{\Gamma}=\langle v, f\rangle+\langle\gamma v, \chi g\rangle_{\Gamma} \geq 0
$$

for $v \in\left(W_{q^{\prime},(1-\chi) \gamma}^{1}\right)^{+}$. Thus $u \in W_{q}^{2} \hookrightarrow W_{q}^{1}$ satisfies (18) so that $u \geq 0$ if (ii) is true. This shows that (ii) implies (iii). 
Now suppose that $A$ is surjective and $(\mathcal{A}, \mathcal{B})$ is inverse positive on $W_{q}^{2}$. Then $A$ is inverse positive. Hence $\lambda_{0}>0$ by (2), so that

$$
0 \in \rho\left(A_{-2}\right)=\rho(A) .
$$

From the second part of (b) we know that $A_{-2}$ is inverse positive. Thus (iii) implies (i). This proves (1).

Proof of Remark 7(b) Replace in the second part of step (b) of the preceding proof the very weak maximum principle by the weak one and $A_{-2}$ by $A_{-1}$. Then it follows that the inverse positivity of $A$ implies the one of $A_{-1}$. Hence from (51) and (18) we deduce that $\lambda_{0}>0$ implies that $(\mathcal{A}, \mathcal{B})$ is inverse positive.

Similarly, by replacing in the beginning of the last paragraph of the preceding proof $A_{-2}$ by $A_{-1}$, we see that (iii) of Theorem 6 implies (ii).

Proof of Theorem 8. It is an easy consequence of Theorems 6 and 39 that

$$
\text { (i) } \Rightarrow \text { (iv) } \Rightarrow \text { (iii) } \Rightarrow \text { (ii). }
$$

Suppose that (ii) is true. Then it is obvious that $(\mathcal{A}, \mathcal{B})$ is inverse positive. Given $f \in L_{q}$, it follows from (ii) that there exists $u^{ \pm} \in W_{q, \mathcal{B}}^{2}$ satisfying $A u^{ \pm}=f^{ \pm}$, where $f^{+}$(resp, $f^{-}$) is the positive (resp. negative) part of $f$. Thus, setting $u:=u^{+}-u^{-} \in W_{q, \mathcal{B}}^{2}$, we see that $A u=f$. Hence $A$ is surjective. Hence we infer from the second part of Theorem $6(2)$ that $\lambda_{0}>0$. Thus (ii) implies (i).

It is obvious from this proof that Theorem 8 remains valid if only the weaker assumption (16) is satisfied, provided assertion (iv) is omitted.

\section{Bounded Domains}

In this section we prove the theorems presented in Sections 6 and 7. We begin with a simple bootstrapping result.

Proposition 43. Let $\Omega$ be bounded and suppose that only condition (16) is satisfied. Suppose also that $u \in W_{q}^{2}$ solves (53). If $q<p<\infty$ and $(f, g) \in L_{p} \times \partial W_{p}^{2}$ then $u \in W_{p}^{2}$.

Proof. Put $r:=p \wedge n q /(n-2 q)$ if $q<n / 2$, and $r:=p$ otherwise. Then $u \in L_{r}$ by Sobolev's embedding theorem. Fix $\omega:=\omega_{r} \in \sigma\left(-A_{(r)}\right)$, where $A_{(r)}$ is the $L_{r}$ realization of $(\mathcal{A}, \mathcal{B})$. Then $(53)$ is equivalent to

$$
(\omega+\mathcal{A}) u=f_{\omega} \text { in } \Omega, \quad \mathcal{B} u=g \text { on } \Gamma,
$$

where

$$
f_{\omega}:=(\omega-\lambda) u+f \in L_{r} \quad \text { and } \quad g \in \partial W_{r}^{2},
$$

thanks to the boundedness of $\Omega$ (and the compactness of $\Gamma$ ). Hence we deduce from Theorem 39(ii) that $u \in W_{r}^{2}$. If $r<p$ we repeat this argument to arrive after finitely many steps at the assertion. 
Proof of Theorem 10 Since $\Omega$ is bounded, embedding (14) is compact. Hence $A$ has a compact resolvent and the assertions concerning $\sigma(A)$ follow from the general theory of linear operators with compact resolvent (e.g., [48]).

Suppose that $\lambda \in \mathbb{C}$,

$$
v \in L_{\infty-}:=\bigcap_{1<p<\infty} L_{p}
$$

and $u \in W_{q}^{2}$ satisfies $A u=\lambda u-v$. Then Proposition 43 implies that $u \in W_{\infty-}^{2}$. Thus, if $\lambda \in \sigma(A)=\sigma_{p}(A)$ and $u \in N_{A}(\lambda)$, there exists $v_{0}, \ldots, v_{m} \in W_{q, \mathcal{B}}^{2}$ satisfying $u_{0}=u$ and

$$
A u_{k}=\lambda u_{k}-v_{k+1}, \quad 0 \leq k \leq m,
$$

where $v_{m+1}=0$. Hence we deduce from what has just been shown, by backwards induction, that $u \in W_{\infty-}^{2}$.

Proposition 44. Let $\Omega$ be bounded and suppose only that condition (16) is satisfied. Also suppose that either $N=1$ or $(a, b)$ is irreducible. If $q>n$ then every positive strict $W_{q}^{2}$ supersolution for $(\mathcal{A}, \mathcal{B})$ is strongly positive.

Proof. Define, as in the proof of Theorem $41,\left(\mathcal{A}^{\triangle}, \mathcal{B}^{\triangle}\right)$ by replacing $(a, b)$ in $(\mathcal{A}, \mathcal{B})$ by $\left(a^{\triangle}, b^{\triangle}\right)$. Then Theorem 35 implies the existence of $\omega>0$ with $\omega>-\lambda_{0}$ such that $\left(\omega+\mathcal{A}^{\triangle}, \mathcal{B}^{\triangle}\right)$ satisfies the strong maximum principle.

Suppose that $u$ is a positive strict $W_{q}^{2}$ supersolution for $(\mathcal{A}, \mathcal{B})$. Then $u$ belongs to $\left(W_{q}^{2}\right)^{+} \backslash\{0\}$ and

$$
(f, g):=(\mathcal{A} u, \mathcal{B} u) \in\left(L_{q} \times \partial W_{q}^{2}\right)^{+} \backslash\{0\} .
$$

Consequently, $u$ satisfies

$$
\left(\omega+\mathcal{A}^{\triangle}\right) u=\left(\omega+a^{\bullet}\right) u+f \text { in } \Omega, \quad \mathcal{B}^{\triangle} u=\chi b^{\bullet} \chi \gamma u+g \text { on } \Gamma .
$$

Note that

$$
\left(\left(\omega+a^{\bullet}\right) u+f, \chi b^{\bullet} \chi \gamma u+g\right)>0,
$$

thanks to $u \geq 0$ and the positivity of $a^{\bullet}, b^{\bullet}$, and the the trace operator.

If $N=1$ then (62) reduces to $(\omega u+f, g)>0$. Hence (61) and the strong maximum principle imply the strong positivity of $u$.

Suppose that $N>1$. Then there exists $s \in\{1, \ldots, N\}$ such that $\left(f^{s}, g^{s}\right)>0$. Hence, by looking at equations number $s$ of system (61), we deduce from Theorem 35 that $u^{s}$ is strongly positive. Fix any $r \in\{1, \ldots, N\}$. Then the irreducibility of $(a, b)$ guarantees the existence of $s_{\rho} \in\{1, \ldots, N\}, 1 \leq \rho \leq k$, with $s_{0}=s$ and $s_{k}=r$ such that

$$
\left(a^{\xi \eta}, \chi^{\xi} b^{\xi \eta} \chi^{\eta}\right)>0 \text { for }(\xi, \eta) \in\left\{\left(s_{1}, s\right), \ldots,\left(r, s_{k-1}\right)\right\} \text {. }
$$

Thus we infer from (62) and $u^{s}(x)>0$ for all $x \in \Omega \cup \Gamma_{1}^{s}$ that component $s_{1}$ of (62) is nonzero. Thus, by looking at equations number $s_{1}$ of system (61) and invoking Theorem 35 once more, we see that $u^{s_{1}}$ is strictly positive. Now we repeat these arguments with $\left(s_{1}, s\right)$ replaced by $\left(s_{2}, s_{1}\right)$, etc. to find that $u^{r}$ is strongly positive. Since this is true for each index $r$ it follows that $u$ is strongly positive. 
Proof of Theorem 12 Since the spectrum of $A$ is independent of $q$ we can assume that $q>n$.

Fix $\omega>-\lambda_{0}$. Then (23) is equivalent to

$$
\mu u=(\omega+A)^{-1} u, \quad \mu:=1 /(\omega+\lambda),
$$

for $\lambda \neq-\omega$. From $\sigma(A) \subset\left[\operatorname{Re} \lambda \geq \lambda_{0}\right]$ it follows that $\operatorname{Re} \mu>0$ if $\lambda \in \sigma(A)$.

Put

$$
E:=\left\{u \in C^{1}(\bar{\Omega}) ;(1-\chi) \gamma u=0\right\} .
$$

Then $E$ is an ordered Banach space whose positive cone has nonempty interior. Indeed, every strongly positive $u$ belongs to $\operatorname{int}\left(E^{+}\right)$. Also set

$$
T:=T_{\omega}:=(\omega+A)^{-1} \mid E .
$$

Then the compactness of the embedding $W_{q, \mathcal{B}}^{2} \hookrightarrow E$ implies that $T$ is a compact endomorphism of $E$. Thus we infer from Theorem 41, Proposition 44, and $E \hookrightarrow L_{q}$ that $T$ is strongly positive, that is,

$$
T\left(E^{+} \backslash\{0\}\right) \subset \operatorname{int}\left(E^{+}\right) .
$$

Consequently, the Krein-Rutman theorem (cf. [1, Theorem 3.2]) implies that the spectral radius $r:=r_{\omega}$ of $T$ is positive and a simple eigenvalue with a positive eigenvector. Moreover, it is the only eigenvalue of $T$ with a positive eigenvector. Clearly, $u \in E$ satisfies $r u=T u$ iff $u \in W_{q, \mathcal{B}}^{2}$ and $u$ satisfies (63) with $\mu:=r$. But this is equivalent to the fact that $u$ is an eigenfunction of $A$ to the eigenvalue

$$
\lambda_{\bullet}:=-\omega+1 / r .
$$

Thus $\sigma(A) \neq \varnothing$ and $\lambda_{\bullet}$ is an eigenvalue of $A$ with a positive eigenfunction $u_{\bullet}$. From (63) we also deduce that $u_{\bullet}$ is a positive strict supersolution of $(\omega+\mathcal{A}, \mathcal{B})$. Hence $u_{\bullet}$ is strongly positive by Proposition 44.

Suppose that there are $u_{0}, \ldots, u_{m} \in W_{q, \mathcal{B}}^{2}$ satisfying

$$
A u_{k}-\lambda_{\bullet} u_{k}=u_{k+1}, \quad 0 \leq k \leq m
$$

where $u_{m+1}:=0$. Then

$$
r u_{k}-T u_{k}=r T u_{k+1}, \quad 0 \leq k \leq m .
$$

Thus, if $m \geq 1$, it follows from $T u_{m}=r u_{m}$ that

$$
r u_{m-1}-T u_{m-1}=r^{2} u_{m} .
$$

Since $r$ is a simple eigenvalue of $T$ there exists $\alpha \in \mathbb{R}$ such that $u_{m}=\alpha u_{\bullet}$. The KreinRutman theorem guarantees also that there exists an eigenvector $\varphi$ of the dual $T^{\prime} \in \mathcal{L}\left(E^{\prime}\right)$ to the eigenvalue $r$ satisfying $\langle\varphi, v\rangle>0$ for $v \in E^{+} \backslash\{0\}$. By applying the functional $\varphi$ to (64) it follows that

$$
r^{2} \alpha\left\langle\varphi, u_{\bullet}\right\rangle=\left\langle r \varphi-T^{\prime} \varphi, u_{m-1}\right\rangle=0
$$

Hence $\alpha=0$. Thus we find by backwards induction that $u_{k}=0$ for $1 \leq k \leq m$, which shows that

$$
N_{A}\left(\lambda_{\bullet}\right)=\operatorname{ker}\left(\lambda_{\bullet}-A\right)
$$


Hence the equivalence of the eigenvalue problem for $A$ with (63) and the simplicity of $r$ imply that $\lambda_{\bullet}$ is a simple eigenvalue of $A$.

Assume that $\lambda \in \rho(-A) \cap \mathbb{R}$ with $\lambda \neq \omega$. Note that $(\lambda+A) u=f$ for $f \in E$ is equivalent to

$$
\left(\frac{1}{\omega-\lambda}-T\right) u=\frac{1}{\omega-\lambda} T f
$$

Suppose that $\omega>\lambda$. Then (65) has for each $f>0$ precisely then a positive solution if $1 /(\omega-\lambda)>r$ (cf. [1, Theorem 3.2(iv)]), that is, if $\lambda>\omega-1 / r=-\lambda_{\bullet}$. Hence, if $(\lambda+A)^{-1} \geq 0$ and $\omega>\lambda$ then it follows that $\lambda>-\lambda_{\bullet}$. Clearly, given any $\lambda \in \mathbb{R}$, we can fix $\omega>\lambda$ such that the above arguments apply. (Note that $\lambda_{\bullet}$ is independent of $\omega$ although $T=T_{\omega}$ and $r=r_{\omega}$ depend on this choice.) This shows that $-\lambda_{\bullet}=s_{+}(-A)$. Hence Theorem 29 implies $-\lambda_{\bullet}=s(-A)=-\lambda_{0}$.

Lastly, suppose that $\lambda \in \sigma(-A) \backslash\left\{\lambda_{0}\right\}$ satisfies $\operatorname{Re} \lambda=\lambda_{0}$. Then it follows from [39, Theorem 2.4] (also see [16, Corollary C-III.2.12] or [25, Theorem 8.14]) that

$$
\lambda_{0}+i k \operatorname{Im} \lambda \in \sigma(A)
$$

for $k \in \mathbb{Z}$. But this contradicts the fact that $\sigma(A)$ is contained in a symmetric sector around the real axis with an angle of opening less than $\pi$, as follows from $A \in \mathcal{H}\left(W_{q, \mathcal{B}}^{2}, L_{q}\right)$. Thus $\lambda_{0}$ is the only eigenvalue of $A$ with $\operatorname{Re} \lambda=\lambda_{0}$.

Proof of Theorem 15 Thanks to Proposition 44 it suffices to show that every positive strict $W_{q}^{2}$ supersolution is strictly positive.

Fix $\omega>\lambda_{0}$ and put

$$
K:=(\omega+A)^{-1} \in \mathcal{L}\left(L_{q}\right) .
$$

Then $K \geq 0$. By repeated application of Proposition 43 we deduce from Proposition 44 that there exists $m \in \mathbb{N}$ such that $K^{j} u$ is strongly positive whenever $j>m$ and $u>0$. Consequently, given $\mu>r(K)$,

$$
K(\mu-K)^{-1} u=\sum_{j=1}^{\infty} \mu^{-j} K^{j} u
$$

is for each $u \in\left(L_{q}^{+}\right) \backslash\{0\}$ a quasi-interior point of $L_{q}^{+}$.

Set $\lambda:=\omega-1 / \mu$ and note that $r(K)=1 /\left(\omega+\lambda_{0}\right)$ implies $\lambda>-\lambda_{0}$. Furthermore,

$$
(\lambda+A)^{-1}=\mu K(\mu-K)^{-1} .
$$

Hence $(\lambda+A)^{-1}$ is strongly irreducible, thus irreducible. Now Theorem 31 implies that $(\lambda+A)^{-1} u$ is for each $\lambda>-\lambda_{0}$ and each $u \in\left(L_{q}^{+}\right) \backslash\{0\}$ a quasi-interior point of $L_{q}^{+}$, hence strictly positive.

Let $u$ be a positive strict $W_{q}^{2}$ supersolution for $(\mathcal{A}, \mathcal{B})$. Set

$$
(f, g):=(\mathcal{A} u, \mathcal{B} u)>0 .
$$


Fix $\omega>\left(-\lambda_{0}\right) \vee 0$ and put $f_{\omega}:=\omega u+f$. Then $f_{\omega}>0$ and the above considerations show that

$$
v:=(\omega+A)^{-1} f_{\omega}
$$

is a strictly positive element of $L_{q}$. Since $g \in\left(\partial W_{q}^{2}\right)^{+}$and

$$
\lambda_{0}(\omega+A)=\omega+\lambda_{0}(A)>0,
$$

it follows from Theorem 8 that there exists a unique $w \in\left(W_{q}^{2}\right)^{+}$satisfying

$$
(\omega+\mathcal{A}) w=0 \text { in } \Omega, \quad \mathcal{B} w=g \text { on } \Gamma .
$$

Since $u=v+w$ we see that $u$ is strictly positive.

Proof of Theorem 13 Suppose that $\lambda_{0}>0$. Then $0 \in \rho(A)$ so that $A$ is surjective. Hence it follows from Theorem $6(1)$ and $(2)$ that $(\mathcal{A}, \mathcal{B})$ is inverse positive and that this equivalent to (ii) and (iii). The inverse positivity of $(\mathcal{A}, \mathcal{B})$ and Proposition 44 imply that $(\mathcal{A}, \mathcal{B})$ satisfies the strong maximum principle. From this we deduce that

$$
\text { (i) } \Rightarrow \text { (ii) } \Rightarrow \text { (iii) } \Rightarrow \text { (iv). }
$$

(iv) $\Rightarrow$ (i) Suppose that $\lambda_{0} \leq 0$ and let $u_{0}$ be a positive eigenfunction of $(\mathcal{A}, \mathcal{B})$. Then

$$
\mathcal{A} u_{0}=\lambda_{0} u_{0} \leq 0 \text { in } \Omega, \quad \mathcal{B} u_{0}=0 \text { on } \Gamma \text {. }
$$

Hence $u_{0} \in W_{\infty-}^{2} \backslash\{0\}$ and the strong maximum principle imply $-u_{0}>0$, which is impossible. Thus $\lambda_{0}>0$.

(i) $\Rightarrow$ (v) Every positive eigenfunction to the eigenvalue $\lambda_{0}$ is a positive strict $W_{q}^{2}$ supersolution, hence a positive strict $L_{q}$ supersolution.

(v) $\Rightarrow$ (i) Recall that $A^{\sharp}=A^{\prime}$, where $A$ is considered as an unbounded operator in $L_{q}$. Hence $\sigma\left(A^{\sharp}\right)=\sigma(A)$. Note that $\left(\mathcal{A}^{\sharp}, \mathcal{B}^{\sharp}\right)$ satisfies condition (7) also and the irreducibility of $(a, b)$ implies the one of $\left(a^{\top}, b^{\top}\right)$. Thus $\lambda_{0}$ is also the principal eigenvalue of $\left(\mathcal{A}^{\sharp}, \mathcal{B}^{\sharp}\right)$ and it has a strongly positive eigenfunction $\varphi_{0} \in W_{\infty-}^{2}$.

Let $u$ be a positive strict $L_{q}$ supersolution for $(\mathcal{A}, \mathcal{B})$. Fix $\omega>-\lambda_{0}$ and put

$$
\widehat{u}:=(\omega+A)^{-1} u .
$$

Then $\widehat{u} \in\left(W_{q, \mathcal{B}}^{2}\right)^{+}$by Theorem 41(iii). From $A_{-2} \supset A$ and

$$
\left(\omega+A_{-2}\right)^{-1} \supset(\omega+A)^{-1}
$$

we deduce that

$$
f:=A \widehat{u}=A_{-2}\left(\omega+A_{-2}\right)^{-1} u=\left(\omega+A_{-2}\right)^{-1}\left(A_{-2} u\right)>0,
$$

where the last inequality sign is also a consequence of Theorem 41(iii) and Theorem 37(iii). Hence $\widehat{u}$ is a positive strict $W_{q}^{2}$ supersolution for $(\mathcal{A}, \mathcal{B})$, and $f$ belongs to $L_{q}^{+} \backslash\{0\}$. Thus the strict positivity of $\varphi_{0}$ implies

$$
0<\left\langle\varphi_{0}, f\right\rangle=\left\langle\varphi_{0}, A \widehat{u}\right\rangle=\left\langle A^{\prime} \varphi_{0}, \widehat{u}\right\rangle=\lambda_{0}\left\langle\varphi_{0}, \widehat{u}\right\rangle
$$

and $\left\langle\varphi_{0}, \widehat{u}\right\rangle>0$. Hence $\lambda_{0}>0$. 
Proof of Remark 14 It suffices to replace in the last paragraph of the preceding proof $A_{-2}$ by $A_{-1}$.

Proof of Theorem 11 Thanks to Theorem 12 we can assume that $N>1$ and $(a, b)$ is reducible. Thus we can also assume that $\left[\left(a^{\bullet}, \chi b^{\bullet} \chi\right)\right]$ has a block triangular structure of the form (24). If the first diagonal block is either one-dimensional or irreducible then we can apply Theorem 12 to the reduced system obtained by setting $u^{M+1}, \ldots, u^{N}$ equal to 0 . This guarantees the existence of a real eigenvalue of $(\mathcal{A}, \mathcal{B})$ with a positive eigenfunction. If $M>2$ and the first diagonal block is reducible we can repeat this argument to arrive at the existence of at least one real eigenvalue of $(\mathcal{A}, \mathcal{B})$ with a positive eigenvector. Thus $\sigma(\mathcal{A}, \mathcal{B}) \neq \varnothing$ and $\lambda_{0}$ is an eigenvalue of $(\mathcal{A}, \mathcal{B})$.

Fix $\omega>-\lambda_{0}$. Then $(\omega+A)^{-1}$ is a positive compact endomorphism of $L_{q}$, and $1 /\left(\omega+\lambda_{0}\right)$ is its spectral radius. Hence the Krein-Rutman theorem (e.g., [1, Theorem 3.1]) guarantees that $(\omega+A)^{-1}$ has a positive eigenfunction $u_{0}$ to the eigenvalue $1 /\left(\omega+\lambda_{0}\right)$. Thus $u_{0}$ is a positive eigenfunction of $A$ to the eigenvalue $\lambda_{0}$.

Finally, if $(\mathcal{A}, \mathcal{B})$ is inverse positive then $A$ is injective. If $A$ is not surjective then $0 \in$ $\sigma(A)$. Thus $\operatorname{ker}(A) \neq\{0\}$ since $\sigma(A)=\sigma_{p}(A)$. This being impossible, $A$ is surjective and the last assertion follows from Theorem 6(2).

\section{Domain perturbations}

In this section we prove Theorems 20 and 22. For this we need some preparation.

We fix $\xi, \eta, \zeta \in[1, \infty]$ satisfying

$$
\frac{1}{\xi} \leq \min \left\{\frac{1}{n}, \frac{1}{q^{\prime}}\right\}
$$

with a strict inequality sign if $q^{\prime}=n$,

$$
\frac{1}{\eta} \leq \min \left\{\frac{2}{n}, \frac{1}{q}+\frac{1}{n}, \frac{1}{q^{\prime}}+\frac{1}{n}\right\}
$$

with a strict inequality sign if either $q=n$ or $q^{\prime}=n$, and

$$
\frac{1}{\zeta} \leq \min \left\{\frac{1}{n-1}, \frac{n}{n-1} \frac{1}{q}, \frac{n}{n-1} \frac{1}{q^{\prime}}\right\}
$$

with a strict inequality sign if either $q=n$ or $q^{\prime}=n$. Then we define a Banach space $\mathbb{E}_{\xi, \eta, \zeta}(\Omega)$ by

$$
\begin{aligned}
\mathbb{E}_{\xi, \eta, \zeta}(\Omega):= & B U C\left(\Omega, \mathbb{R}_{\text {diag }}^{N \times N}\right)^{n \times n} \times\left(L_{\xi}+L_{\infty}\right)\left(\Omega, \mathbb{R}_{\text {diag }}^{N \times N}\right)^{n} \\
& \times\left(L_{\eta}+L_{\infty}\right)\left(\Omega, \mathbb{R}^{N \times N}\right) \times L_{\zeta}\left(\Gamma, \mathbb{R}^{N \times N}\right) \times C\left(\Gamma, \mathbb{R}_{\text {diag }}^{N \times N}\right) .
\end{aligned}
$$

Given

$$
\alpha:=\left(\left[a_{j k}\right]^{N \times N},\left(a_{1}, \ldots, a_{n}\right), a, b, \chi\right) \in \mathbb{E}_{\xi, \eta, \zeta}(\Omega)
$$


we put

$$
\mathfrak{a}(\alpha)(v, u):=\left\langle\partial_{j} v, a_{j k} \partial_{k} u\right\rangle+\left\langle v, a_{j} \partial_{j} u+a u\right\rangle+\langle\gamma v, \chi b \gamma u\rangle_{\Gamma}
$$

for $(v, u) \in W_{q^{\prime}}^{1} \times W_{q}^{1}$.

For Banach spaces $E$ and $F$ we write $\mathcal{L}(E, F ; \mathbb{R})$ for the Banach space of all continuous bilinear maps $E \times F \rightarrow \mathbb{R}$, endowed with its usual norm.

Given $\mathfrak{b} \in \mathcal{L}(E, F ; \mathbb{R})$, let $B_{\mathfrak{b}}$ be the unique linear operator in $\mathcal{L}\left(F, E^{\prime}\right)$ satisfying

$$
\left\langle B_{\mathfrak{b}} f, e\right\rangle_{E}=\mathfrak{b}(e, f), \quad(e, f) \in E \times F .
$$

It follows that the map

$$
\mathcal{L}(E, F ; \mathbb{R}) \rightarrow \mathcal{L}\left(F, E^{\prime}\right), \quad \mathfrak{b} \mapsto B_{\mathfrak{b}}
$$

is a linear isometry.

Lemma 45. The map

$$
\mathbb{E}_{\xi, \eta, \zeta}(\Omega) \rightarrow \mathcal{L}\left(W_{q^{\prime}}^{1}, W_{q}^{1} ; \mathbb{R}\right), \quad \alpha \mapsto \mathfrak{a}(\alpha),
$$

is well-defined, linear, and continuous.

Proof. This follows from Sobolev embeddings and the trace theorem.

Suppose now that $\Omega$ is bounded. Let $\underset{\sim}{\Omega}$ be a bounded $C^{2}$ domain in $\mathbb{R}^{n}$ with boundary $\underset{\sim}{\Gamma}$ and trace operator $\underset{\sim}{\gamma}$. Also suppose that $\underset{\sim}{\chi}$ is a boundary identification map for $\underset{\sim}{\Omega}$. We set

$$
X:=\bar{\Omega} \quad \text { and } \quad Y:=\bar{\Omega}
$$

and denote by $\left(x^{1}, \ldots, x^{n}\right)$ and $\left(y^{1}, \ldots, y^{n}\right)$ the standard Euclidean coordinates of $X$ and $Y$, respectively. Then $X$ and $Y$ are compact oriented $n$-dimensional Riemannian $C^{2}$ manifolds with boundary and the standard Euclidean metric

$$
(\cdot \mid \cdot)_{X}:=d x^{j} \otimes d x^{j} \quad \text { and } \quad(\cdot \mid \cdot)_{Y}:=d y^{j} \otimes d y^{j},
$$

respectively.

Also suppose that $\varphi: X \rightarrow Y$ is an orientation preserving $C^{2}$ diffeomorphism satisfy$\operatorname{ing} \varphi_{\partial}^{*} \chi \sim \sim$. Then



with inverse

$$
\varphi_{*}:=\left(\varphi^{-1}\right)^{*} .
$$

Indeed, this is easily verified if $j \in\{0,1\}$, and follows by duality if $j=-1$. 
Put

$$
(\cdot \mid \cdot)_{M}:=\varphi^{*}\left(d y^{j} \otimes d y^{j}\right)=g_{j k} d x^{j} \otimes d x^{k}
$$

where

$$
g_{j k}:=\left(\frac{\partial \varphi}{\partial x^{j}} \mid \frac{\partial \varphi}{\partial x^{k}}\right)_{X}, \quad 1 \leq j, k \leq n
$$

Then $M:=\left(X,(\cdot \mid \cdot)_{M}\right)$ is an oriented $n$-dimensional Riemannian $C^{2}$ manifold with boundary, and $\varphi$ is an orientation preserving $C^{2}$ diffeomorphism from $M$ onto $Y$. The volume element, $\omega_{M}$, of $M$ is given by

$$
\omega_{M}=\varphi^{*} \omega_{Y}=\sqrt{g} d x^{1} \wedge \cdots \wedge d x^{n}
$$

with

$$
g:=g_{\varphi}:=\operatorname{det}\left[g_{j k}\right] .
$$

Since $M$ and $Y$ are compact, there exists a constant $\kappa \geq 1$ such that $\kappa^{-1} \leq \sqrt{g} \leq \kappa$. Hence

$$
L_{p}\left(M, \mathbb{R}^{N}\right):=L_{p}\left(M, \operatorname{vol}_{M}, \mathbb{R}^{N}\right) \doteq L_{p}, \quad 1 \leq p \leq \infty
$$

where $\operatorname{vol}_{M}$ is the Lebesgue volume measure on $M$ induced by $\omega_{M}$.

Moreover, $\varphi_{\partial}$ is an orientation preserving $C^{2}$ diffeomorphism from $\partial X=\Gamma$ onto $\partial Y=\underset{\sim}{\Gamma}$, and $\partial M$ is an $(n-1)$-dimensional Riemannian $C^{2}$ manifold, oriented by means of the outer unit normal, and with the volume element

$$
\omega_{\partial M}=\varphi_{\partial}^{*} \omega_{\partial Y}
$$

There exists a unique $J \in C^{1}(\Gamma, \mathbb{R})$ satisfying

$$
\omega_{\partial M}=J \omega_{\partial X},
$$

the Jacobian of $\varphi_{\partial}$. Consequently,

$$
\operatorname{vol}_{\partial M}=J d \sigma .
$$

By means of local coordinates and the compactness of $\Gamma$ one finds a constant $\kappa_{1} \geq 1$ such that $\kappa_{1}^{-1} \leq J \leq \kappa_{1}$. Hence

$$
L_{p}\left(\partial M, \mathbb{R}^{N}\right):=L_{p}\left(\partial M, \operatorname{vol}_{\partial M}, \mathbb{R}^{N}\right) \doteq L_{p}(\Gamma), \quad 1 \leq p \leq \infty .
$$

Given a Riemannian manifold $R$, we denote by $\mathcal{V}_{p}^{j}(R)$ the Banach space of all $W_{p}^{j}$ vector fields on $R$ for $1 \leq p \leq \infty$ and $j=0,1$, and $\operatorname{grad}_{R}$ is the gradient operator on $R$. We identify $\vec{w} \in \mathcal{V}_{p}^{j}(Y)$ with

$$
\left(w^{1}, \ldots, w^{n}\right) \in W_{p}^{j}\left(\underset{\sim}{\Omega}, \mathbb{R}^{n}\right)
$$

by setting

$$
\vec{w}=w^{j} \partial / \partial y^{j} .
$$

Hence $\varphi_{*} \vec{v}$, the push forward of $\vec{v} \in \mathcal{V}^{j}(M)$, is given by

$$
\varphi_{*} \vec{v}=\varphi_{*}(\partial \varphi)\left(\vec{v} \circ \varphi^{-1}\right),
$$

where $\partial \varphi \in C^{1}\left(\bar{\Omega}, \mathbb{R}^{n \times n}\right)$ is the derivative of $\varphi$. It follows that

$$
\varphi_{*} \in \mathcal{L} \operatorname{is}\left(\mathcal{V}_{p}^{j}(M), \mathcal{V}_{p}^{j}(Y)\right), \quad 1 \leq p \leq \infty, \quad j=0,1,
$$


and that its inverse is given by

$$
\varphi^{*}:=\left(\varphi^{-1}\right)_{*}=\left(\vec{w} \mapsto \varphi^{*}\left(\partial \varphi^{-1}\right)(\vec{w} \circ \varphi)\right) .
$$

Since

$$
\varphi^{*}\left(\varphi^{-1}\right)=\varphi^{-1} \circ \varphi=\operatorname{id}_{\Omega}
$$

the chain rule implies

$$
\varphi^{*}\left(\partial \varphi^{-1}\right) \partial \varphi=1
$$

Consequently,

$$
\varphi^{*} \vec{w}=(\partial \varphi)^{-1}(\vec{w} \circ \varphi), \quad w \in \mathcal{V}_{p}^{j}(Y), \quad 1 \leq p \leq \infty, \quad j=0,1 .
$$

Also note that

$$
\left[g_{j k}\right]=(\partial \varphi)^{\top} \partial \varphi
$$

Hence

$$
\left[g^{j k}\right]:=\left[g_{j k}\right]^{-1}=(\partial \varphi)^{-1}\left((\partial \varphi)^{-1}\right)^{\top}
$$

where we identify $\mathbb{R}^{n \times n}$ and $\mathcal{L}\left(\mathbb{R}^{n}\right)$ by means of the standard basis. Thus, using the representation of $\operatorname{grad}_{M}$ in the $x$-coordinates, it follows that

$$
\operatorname{grad}_{M} f=g^{j k} \frac{\partial f}{\partial x^{k}} \frac{\partial}{\partial x^{j}}=(\partial \varphi)^{-1}\left((\partial \varphi)^{-1}\right)^{\top} \operatorname{grad} f, \quad f \in \mathcal{V}_{p}^{1}(\Omega),
$$

for $1 \leq p \leq \infty$, where we write $\operatorname{grad}:=\operatorname{grad}_{X}$ and, later, $(\cdot \mid \cdot):=(\cdot \mid \cdot)_{X}$.

Observe that

$$
(\vec{v} \mid \vec{w})_{M}=\varphi^{*}\left(\varphi_{*} \vec{v} \mid \varphi_{*} \vec{w}\right)_{Y}, \quad(\vec{v}, \vec{w}) \in \mathcal{V}_{p^{\prime}}^{j}(M) \times \mathcal{V}_{p}^{j}(M), \quad j=0,1,
$$

for $1 \leq p \leq \infty$. Thus, given $f \in W_{p}^{1}(\underset{\sim}{\Omega}, \mathbb{R})$ and $\vec{v} \in \mathcal{V}_{p^{\prime}}^{0}(\Omega)$, the definition of the gradient implies

$$
\begin{aligned}
\left(\operatorname{grad}_{M}\left(\varphi^{*} f\right) \mid \vec{v}\right)_{M} & =d\left(\varphi^{*} f\right) \vec{v}=\varphi^{*}(d f) \vec{v}=\varphi^{*}\left(d f\left(\varphi_{*} \vec{v}\right)\right) \\
& =\varphi^{*}\left(\operatorname{grad}_{Y} f \mid \varphi_{*} \vec{v}\right)_{Y}=\left(\varphi^{*} \operatorname{grad}_{Y} f \mid \vec{v}\right)_{M},
\end{aligned}
$$

using standard properties of the pull back and push forward operators. (We refer to [10] for the theory of differential forms and vector fields as well as the elementary Riemannian geometry used in this section). Hence

$$
\operatorname{grad}_{M}=\varphi^{*} \circ \operatorname{grad}_{Y} \circ \varphi_{*} \in \mathcal{L}\left(\mathcal{V}_{p}^{1}(M), \mathcal{V}_{p}^{0}(M)\right), \quad 1 \leq p \leq \infty
$$

Now suppose that

$$
\underset{\sim}{\alpha}:=\left(\left[\underset{\sim}{a_{j k}}\right],\left(\underset{\sim}{a_{1}}, \ldots, \underset{\sim}{a}\right), \underset{\sim}{a}, \underset{\sim}{b}, \underset{\sim}{\chi}\right) \in \mathcal{E}(\underset{\sim}{\Omega})
$$

such that

$$
\varphi_{\partial}^{*} \underset{\sim}{\chi}=\chi
$$

where $\chi$ is a fixed boundary identification map for $\Omega$. Put

$$
\underset{\sim}{\mathfrak{a}}:=\mathfrak{a}(\underset{\sim}{\alpha})
$$


and observe that

$$
\underset{\sim}{\mathfrak{a}}=\sum_{r=1}^{N} \underset{\sim}{\mathfrak{a}^{r}}
$$

with

$$
\begin{aligned}
& {\underset{\sim}{\mathfrak{a}}}^{r}(\underset{\sim}{v}, \underset{\sim}{u})=\int_{\Omega}\left\{\left(\operatorname{grad}_{Y} \underset{\sim}{v^{r}} \mid \underset{\sim}{\boldsymbol{a}^{r}} \operatorname{grad}_{Y} \underset{\sim}{u}\right)_{Y}+{\underset{\sim}{v}}^{r}\left[\left({\underset{\sim}{a}}^{r} \mid \operatorname{grad}_{Y}{\underset{\sim}{u}}^{r}\right)_{Y}+\sum_{s=1}^{N} \underset{\sim}{a^{r s}}{\underset{\sim}{u}}^{s}\right]\right\} d y
\end{aligned}
$$

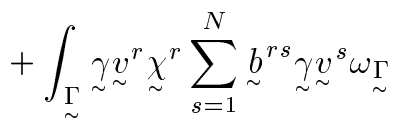

for $1 \leq r \leq N$ and

$$
(\underset{\sim}{v}, \underset{\sim}{u}) \in W_{q^{\prime}}^{1}\left(Y, \mathbb{R}^{N}\right) \times W_{q}^{1}\left(Y, \mathbb{R}^{N}\right),
$$

where

$$
\underset{\sim}{\underset{a}{r}}:=\left[\underset{\sim}{\underset{\sim}{a}}{ }_{j k}^{r}\right], \quad \underset{\sim}{\vec{a}} r:=\left(\underset{\sim}{a}{ }_{1}^{r}, \ldots, \underset{\sim}{\underset{\sim}{a}}{ }_{n}^{r}\right), \quad 1 \leq r \leq N .
$$

Note that (73), (78), and (79), imply

$$
\left(\operatorname{grad}_{Y}\left(\varphi_{*} v^{r}\right) \mid \underset{\sim}{\boldsymbol{a}} \operatorname{grad}_{Y}\left(\varphi_{*} u^{r}\right)\right)_{Y}=\varphi_{*}\left(\operatorname{grad}_{M} v^{r} \mid \varphi^{*}\left(\underset{\sim}{\boldsymbol{a}} \varphi_{*}\left(\operatorname{grad}_{M} u^{r}\right)\right)\right)_{M}
$$

for $(v, u) \in W_{q^{\prime}}^{1} \times W_{q}^{1}$. From (75)-(77) we deduce that

$$
\varphi^{*}\left(\underset{\sim}{\boldsymbol{a}} \varphi_{*}\left(\operatorname{grad}_{M} u^{r}\right)\right)=(\partial \varphi)^{-1}\left(\varphi^{*} \underset{\sim}{\boldsymbol{a}}\left((\partial \varphi)^{-1}\right)^{\top} \operatorname{grad} u^{r} .\right.
$$

By inserting this in (80) and recalling (71) and the representation (77) of $\operatorname{grad}_{M}$ in the $x$-coordinates we arrive at

$\left(\operatorname{grad}_{Y}\left(\varphi_{*} v^{r}\right) \mid \underset{\sim}{\boldsymbol{a}^{r}} \operatorname{grad}_{Y}\left(\varphi_{*} u^{r}\right)\right)_{Y}=\varphi_{*}\left(\operatorname{grad} v^{r} \mid(\partial \varphi)^{-1}\left(\varphi^{*} \underset{\sim}{\boldsymbol{a}}\right)\left((\partial \varphi)^{-1}\right)^{\top} \operatorname{grad} u^{r}\right)$.

Similarly,

$$
\left({\underset{\sim}{a}}^{r} \mid \operatorname{grad}_{Y}\left(\varphi_{*} u^{r}\right)\right)_{Y}=\varphi_{*}\left((\partial \varphi)^{-1}\left(\vec{a}^{r} \circ \varphi\right) \mid \operatorname{grad} u^{r}\right) .
$$

Now, using (72), (74), (80), (81), and the (global) transformation theorem (e.g., [10, Theorem XII.2.3]), we see that

$$
\begin{aligned}
\underset{\sim}{\mathfrak{a}^{r}\left(\varphi_{*} v, \varphi_{*} u\right)=} \int_{\Omega} & \left\{\left(\operatorname{grad} v^{r} \mid(\partial \varphi)^{-1}\left(\varphi^{*} \underset{\sim}{\boldsymbol{a}^{r}}\left((\partial \varphi)^{-1}\right)^{\top} \operatorname{grad} u^{r}\right)\right.\right. \\
& \left.+v^{r}\left[\left((\partial \varphi)^{-1}\left(\underset{\sim}{\vec{a}}{ }^{r} \circ \varphi\right) \mid \operatorname{grad} u^{r}\right)+\sum_{s=1}^{N}\left(\varphi^{*} \underset{\sim}{a^{r s}}\right) u^{s}\right]\right\} \sqrt{g} d x \\
& +\int_{\Gamma} \gamma v^{r} \chi^{r} \sum_{s=1}^{N}\left(\varphi_{\partial}^{*}{\underset{\sim}{r}}^{r s}\right) \gamma u^{s} J d \sigma
\end{aligned}
$$

for $1 \leq r \leq N$ and $(v, u) \in W_{q^{\prime}}^{1} \times W_{q}^{1}$.

Set

$$
\underset{\sim}{a_{j k, \varphi}^{r}}:=\sqrt{g}\left((\partial \varphi)^{-1}\left(\varphi^{*} \underset{\sim}{\boldsymbol{a}^{r}}\right)\left((\partial \varphi)^{-1}\right)^{\top}\right)_{j k}
$$


and

$$
\underset{\sim}{a} a_{j, \varphi}^{r}:=\sqrt{g}(\partial \varphi)^{-1}(\underset{\sim}{a} \circ \varphi)
$$

for $1 \leq j, k \leq n$, as well as

$$
\underset{\sim}{a}{ }_{\varphi}:=\sqrt{g} \varphi_{\sim}^{*} \underset{\sim}{a}, \quad \underset{\sim}{b}:=J \varphi_{\partial}^{*} \underset{\sim}{b} .
$$

Then

$$
\underset{\sim}{\alpha}:=\left([\underset{\sim}{a} j k, \varphi],\left(\underset{\sim}{a} a_{1, \varphi}, \ldots, \underset{\sim}{a} n, \varphi\right), \underset{\sim}{a}, \underset{\sim}{b}, \chi\right) \in \mathcal{E}(\Omega)
$$

Also set

$$
\varphi^{*} \underset{\sim}{\mathfrak{a}}(u, v):=\underset{\sim}{\mathfrak{a}}\left(\varphi_{*} u, \varphi_{*} v\right), \quad(v, u) \in W_{q^{\prime}}^{1} \times W_{q}^{1} .
$$

Then it follows from (82) that

$$
\varphi^{*} \mathfrak{\sim}=\mathfrak{a}(\underset{\sim}{\alpha}),
$$

that is, $\varphi^{*} \mathfrak{\sim}$ is the Dirichlet form of $\left(\mathcal{A}\left(\alpha_{\varphi}\right), \mathcal{B}\left(\alpha_{\varphi}\right)\right)$.

Given $(v, u) \in L_{q^{\prime}} \times L_{q}$, we deduce from (72) and the transformation theorem that

$$
\begin{aligned}
\left\langle\varphi_{*} v, \varphi_{*} u\right\rangle_{L_{q}\left(Y, \mathbb{R}^{N}\right)} & =\int_{Y} \varphi_{*} v \cdot \varphi_{*} w \omega_{Y} \\
& =\int_{Y} \varphi_{*}\left(v \cdot w \sqrt{g} d x^{1} \wedge \cdots \wedge d x^{n}\right) \\
& =\int_{\Omega} v \cdot w \sqrt{g} d x=\langle v, \sqrt{g} w\rangle .
\end{aligned}
$$

Thus, thanks to (70) and a density argument,

$$
\left\langle\varphi_{*} v, \varphi_{*} w\right\rangle_{W_{q,(1-\chi}^{-1} \underset{\sim}{-1} \underset{\sim}{\sim} \underset{\sim}{\left(\Omega, \mathbb{R}^{N}\right)}}=\langle v, \sqrt{g} w\rangle_{W_{q,(1-\chi) \gamma}^{-1}}, \quad(v, w) \in W_{q^{\prime},(1-\chi) \gamma}^{1} \times W_{q,(1-\chi) \gamma}^{1} .
$$

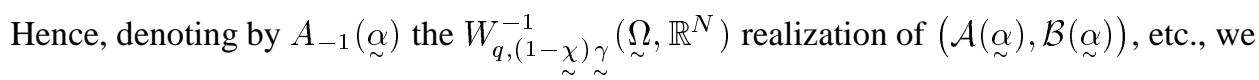
obtain from (87) and $\underset{\sim}{\mathfrak{a}}=\mathfrak{a}(\underset{\sim}{\alpha})$ that

$$
\begin{aligned}
& \left\langle v, A_{-1}(\underset{\sim}{\alpha}) u\right\rangle_{W_{q,(1-\chi) \gamma}^{-1}}=\mathfrak{a}(\underset{\sim}{\alpha})(v, u)=\varphi^{*} \mathfrak{a}(\underset{\sim}{\alpha})(v, u)=\mathfrak{a}(\underset{\sim}{\alpha})\left(\varphi_{*} v, \varphi_{*} u\right) \\
& =\left\langle\varphi_{*} v, A_{-1}(\underset{\sim}{\alpha}) \varphi_{*} u\right\rangle_{\left.W_{q,(1-\chi}^{-1}\right) \underset{\sim}{\sim}\left(\Omega, \mathbb{R}^{N}\right)} \\
& =\left\langle\varphi_{*} v, \varphi_{*}\left(\varphi^{*} \circ A_{-1}(\underset{\sim}{\alpha}) \varphi_{*} u\right)\right\rangle_{\left.W_{q,(1-\chi \sim \sim}^{-1}\right) \chi} \\
& =\left\langle v, \sqrt{g}\left(\varphi^{*} \circ A_{-1}(\underset{\sim}{\alpha}) \circ \varphi_{*}\right) u\right\rangle_{W_{q,(1-\chi) \gamma}^{-1}}
\end{aligned}
$$

for

This shows that

$$
(v, u) \in W_{q^{\prime},(1-\chi) \gamma}^{1} \times W_{q,(1-\chi) \gamma}^{1} .
$$

$$
\varphi^{*} \circ A_{-1}(\underset{\sim}{\alpha}) \circ \varphi_{*}=\frac{1}{\sqrt{g}} A_{-1}(\underset{\sim}{\alpha}) .
$$

Next we give an easy proof of a perturbation theorem for simple eigenvalues. 
Theorem 46. Let $E_{0}$ and $E_{1}$ be Banach spaces such that $E_{1} \hookrightarrow E_{0}$. Suppose that $X$ is a topological space and

$$
B(\cdot) \in C\left(X, \mathcal{L}\left(E_{1}, E_{0}\right)\right)
$$

Also suppose that $x_{0} \in X$ and $\beta_{0}$ is an isolated simple eigenvalue of $B_{0}:=B\left(x_{0}\right)$. Then, given a corresponding eigenvector $e_{0}$ of norm 1 in $E_{1}$, there exist an open neighborhood $U \times V \times W$ of $\left(x_{0}, \beta_{0}, e_{0}\right)$ in $X \times \mathbb{C} \times E_{1}$ and a map $(\beta(\cdot), e(\cdot)) \in C(U, V \times W)$ such that

$$
\left(\beta\left(x_{0}\right), e\left(x_{0}\right)\right)=\left(\beta_{0}, e_{0}\right), \quad\|e(x)\|_{E_{1}}=1,
$$

and

$$
B(x) e(x)=\beta(x) e(x)
$$

for $x \in U$. Furthermore, $\beta(x)$, resp. $e(x)$, is, for $x \in U$, the only eigenvalue, resp. eigenvector, of $B(x)$ in $V$, resp. $W$.

Proof. Since $\beta_{0}$ is an isolated simple eigenvalue of $B_{0}$, it follows that $B_{0}$, considered as a linear operator in $E_{0}$, has a nonempty resolvent set. Consequently, $B_{0}$ is closed in $E_{0}$. Thus spectral theory implies that

$$
E_{0}=\mathbb{C}_{e_{0}} \oplus \operatorname{im}\left(\beta_{0}-B_{0}\right) .
$$

Furthermore, $B_{0}^{\prime}$, the dual of $B_{0}$ (in $E_{0}$ ), has $\beta_{0}$ as a simple eigenvalue as well, and there exists a unique eigenvector $e_{0}^{\prime}$ of $B_{0}^{\prime}$ to the eigenvalue $\beta_{0}$ satisfying $\left\langle e_{0}^{\prime}, e_{0}\right\rangle=1$. In addition,

$$
\operatorname{im}\left(\beta_{0}-B_{0}\right)=\left\{e \in E_{0} ;\left\langle e_{0}^{\prime}, e\right\rangle=0\right\}
$$

(e.g., [48, Sections III.6.5 and III.6.6]).

Now we define a $C^{1}$ map

$$
f: \mathcal{L}\left(E_{1}, E_{0}\right) \times\left(\mathbb{C} \times E_{1}\right) \rightarrow \mathbb{C} \times E_{0}
$$

by

$$
f(B,(\beta, e)):=\left(\left\langle e_{0}^{\prime}, e\right\rangle-1, B e-\beta e\right)
$$

Note that

$$
f\left(B_{0},\left(\beta_{0}, e_{0}\right)\right)=0
$$

and

$$
\partial_{2} f\left(B_{0},\left(\beta_{0}, e_{0}\right)\right)(\beta, e)=\left(\left\langle e_{0}^{\prime}, e\right\rangle, B e-\beta_{0} e-\beta e_{0}\right), \quad(\beta, e) \in \mathbb{C} \times E_{1} .
$$

Hence $\partial_{2} f\left(B_{0},\left(\beta_{0}, e_{0}\right)\right)(\beta, e)=0$ implies $\left\langle e_{0}^{\prime}, e\right\rangle=0$, that is,

$$
e \in \operatorname{im}\left(\beta_{0}-B_{0}\right) \text { and } B e-\beta_{0} e=\beta e_{0} .
$$

Thus we deduce from (89) that

$$
(\beta, e)=0,
$$

which shows that the linear operator $\partial_{2} f\left(B_{0},\left(\beta_{0}, e_{0}\right)\right)$ is injective. From (89) we also infer that this map is surjective. Hence

$$
\partial_{2} f\left(B_{0},\left(\beta_{0}, e_{0}\right)\right) \in \mathcal{L i s}\left(\mathbb{C} \times E_{1}, \mathbb{C} \times E_{0}\right)
$$


by the open mapping theorem. Now the implicit function theorem guarantees the existence of an open neighborhood $\mathrm{B} \times V \times W$ of $\left(B_{0}, \beta_{0}, e_{0}\right)$ in $\mathcal{L}\left(E_{1}, E_{0}\right) \times \mathbb{C} \times E_{1}$ and a map $h \in C^{1}(\mathrm{~B}, V \times W)$ such that

$$
(B,(\beta, e)) \in \mathrm{B} \times V \times W \text { and } f(B,(\beta, e))=0 \quad \text { iff } \quad B \in \mathrm{B} \text { and }(\beta, e)=h(B) .
$$

Since $B(\cdot) \in C\left(X, \mathcal{L}\left(E_{1}, E_{0}\right)\right)$ there exists an open neighborhood $U$ of $x_{0}$ in $X$ such that $B(U) \subset \mathrm{B}$. Thus the assertion follows by setting

$$
\left(\beta(x), e_{1}(x)\right):=h(B(x)), \quad e(x):=e_{1}(x) /\left\|e_{1}(x)\right\|_{E_{1}}
$$

for $x \in U$.

This proof is a modification and an extension to the infinite dimensional case of the one of [6, Proposition (26.24)]. An alternative proof of Theorem 46 can also be derived from the general perturbation results in [48, Section IV.3.4].

After these preparations we are ready to derive the theorems stated in Section 9. In fact, instead of Theorem 20 we prove the following more precise version.

Theorem 47. Let the hypotheses of Theorem 20 be satisfied with the exception that it is assumed that

$$
\varphi_{i}^{*} \alpha_{i} \rightarrow \alpha \quad \text { in } \mathbb{E}_{\xi, \eta, \zeta}(\Omega) .
$$

Then the assertions of Theorem 20 are valid, except that $\varphi_{i}^{*} u_{i} \rightarrow u$ in $W_{q}^{1}$.

Proof. It follows from $\varphi_{i} \rightarrow \operatorname{id}_{\Omega}$ in $C^{1}\left(\bar{\Omega}, \mathbb{R}^{n}\right)$ that $\partial \varphi_{i} \rightarrow 1$ in $C\left(\bar{\Omega}, \mathbb{R}^{n \times n}\right)$ and $\sqrt{g_{\varphi_{i}}} \rightarrow 1$ in $C(\bar{\Omega}, \mathbb{R})$ as well as $J_{\varphi_{i}} \rightarrow 1$ in $C(\Gamma, \mathbb{R})$. Hence we deduce from (90) and (83)-(86) that

$$
\alpha_{i, \varphi_{i}} \rightarrow \alpha \quad \text { in } \quad \mathbb{E}_{\xi, \eta, \zeta}(\Omega) .
$$

This implies

$$
\varphi_{i, \partial}^{*} \chi_{i}=\chi
$$

for all sufficiently large $i$. Thus we can assume that (92) is true for all $i \in \mathbb{N}$. Now we infer from (91), (92), and Lemma 45 that

$$
\mathfrak{a}\left(\alpha_{i, \varphi_{i}}\right) \rightarrow \mathfrak{a}(\alpha) \quad \text { in } \mathcal{L}\left(W_{q^{\prime},(1-\chi) \gamma}^{1}, W_{q,(1-\chi) \gamma}^{1} ; \mathbb{R}\right) .
$$

Consequently, (69) and (88) imply, setting $(\mathcal{A}, \mathcal{B}):=(\mathcal{A}(\alpha), \mathcal{B}(\alpha))$,

$$
C_{i}:=\varphi_{i}^{*} \circ A_{-1}\left(\alpha_{i}\right) \circ \varphi_{i, *} \rightarrow A_{-1} \quad \text { in } \mathcal{L}\left(W_{q, \mathcal{B}}^{1}, W_{q, \mathcal{B}}^{-1}\right) .
$$

Since, by (70),

with inverse $\varphi_{i, *}$, we see that

$$
\varphi_{i}^{*} \in \mathcal{L} \operatorname{is}\left(W_{q, \mathcal{B}\left(\alpha_{i}\right)}^{j}\left(\Omega_{i}, \mathbb{R}^{N}\right), W_{q, \mathcal{B}}^{j}\right), \quad j= \pm 1,
$$

and

$$
\sigma\left(C_{i}\right)=\sigma\left(A_{-1}\left(\alpha_{i}\right)\right)
$$

for $i \in \mathbb{N}$.

$$
\operatorname{ker}\left(\lambda-C_{i}\right)=\varphi_{i}^{*}\left(\operatorname{ker}\left(\lambda-A_{-1}\left(\alpha_{i}\right)\right)\right), \quad \lambda \in \mathbb{C},
$$


Set

$$
X:=\left\{\left(\operatorname{id}_{\bar{\Omega}}, \alpha\right)\right\} \cup\left\{\left(\varphi_{i}, \alpha_{i}\right) ; i \in \mathbb{N}\right\} .
$$

Endow $X$ with the topology induced by $C^{1}\left(\bar{\Omega}, \mathbb{R}^{n}\right) \times \mathbb{E}_{\xi, \eta, \zeta}(\Omega)$. Put

$$
E_{0}:=W_{q, \mathcal{B}}^{-1} \quad \text { and } \quad E_{1}:=W_{q, \mathcal{B}}^{1},
$$

and define $B(x) \in \mathcal{L}\left(E_{1}, E_{0}\right)$ by

$$
B(x):=\left\{\begin{array}{lll}
A_{-1} & \text { if } & x=x_{0}:=\left(\mathrm{id}_{\Omega}, \alpha\right), \\
C_{i} & \text { if } & x \neq x_{0} .
\end{array}\right.
$$

Then (93) implies $B(\cdot) \in C\left(X, \mathcal{L}\left(E_{1}, E_{0}\right)\right)$. Furthermore, Theorem 12 guarantees that $\beta_{0}:=\lambda_{0}(\mathcal{A}, \mathcal{B})$ is a simple isolated eigenvalue of $B\left(x_{0}\right)$ and that $e_{0}:=u$ is a corresponding eigenvector. Thus Theorem 46 guarantees the existence of an eigenvalue $\beta_{i}$ of $C_{i}$ and corresponding eigenvector $e_{i}$ such that $\beta_{i} \rightarrow \beta$ and $e_{i} \rightarrow e_{0}$ in $W_{q, \mathcal{B}}^{1}$ with $\left\|e_{i}\right\|_{W_{q}^{1}}=1$. From the upper semicontinuity of the spectrum (e.g., [48, Theorem IV.3.1]) it easily follows that

$$
\beta_{i}=\lambda_{0}\left(\mathcal{A}\left(\alpha_{i}\right), \mathcal{B}\left(\alpha_{i}\right), \Omega_{i}\right)
$$

for all sufficiently large $i$. Finally, $u \geq 0$ implies $e_{i}=\varphi_{i}^{*} u_{i}$, where $u_{i}$ is a positive eigenfunction of $\left(\mathcal{A}\left(\alpha_{i}\right), \mathcal{B}\left(\alpha_{i}\right)\right)$, since $\lambda_{0}\left(\mathcal{A}\left(\alpha_{i}\right), \mathcal{B}\left(\alpha_{i}\right), \Omega_{i}\right)$ is also a simple eigenvalue of $\left(\mathcal{A}\left(\alpha_{i}\right), \mathcal{B}\left(\alpha_{i}\right)\right)$ by Theorem 12 .

Clearly, $(r, s, t)$, defined in (31), is an admissible choice for $(\xi, \eta, \zeta)$ if $q=2$. Hence Theorem 20 is a particular case of Theorem 47.

In order to prove Theorem 22 we prepare the following technical lemma, where we use the notations introduced in Section 9.

Lemma 48. Suppose that $\beta \in C^{2}(\Gamma,(-\rho, \rho))$ and $\|\beta\|_{\infty} \leq \rho / 3$. Then there exists an orientation preserving $C^{2}$ diffeomorphism $\varphi: \bar{\Omega} \rightarrow \bar{\Omega}_{\beta}$ satisfying $\varphi \mid \Gamma=\psi_{\beta}$ and $\varphi(x)=x$ for $x \in \Omega$ with $\operatorname{dist}(x, \Gamma) \geq 2\|\beta\|_{\infty}$.

Proof. Denote by $\widetilde{\beta} \in C^{2}(\Gamma,(-1,1))$ the image of $\beta$ under the $C^{2}$ diffeomorphism $h$ of (32) and let $\widetilde{\Gamma}$ be the graph of $\widetilde{\beta}$. Then $\widetilde{\Gamma}$ is an oriented $(n-1)$-dimensional $C^{2}$ manifold lying in $\Gamma \times[-\widetilde{\delta}, \widetilde{\delta}]$, where $\widetilde{\delta}:=\|\widetilde{\beta}\|_{\infty}<1 / 3$. Fix $\sigma \in C^{2}(\mathbb{R},[0,1])$ satisfying $\sigma(s)=1$ for $|s| \leq \widetilde{\delta}$ and $\sigma(s)=0$ for $|s| \geq 2 \widetilde{\delta}$, and being strictly decreasing on $(\widetilde{\delta}, 2 \widetilde{\delta})$ and even. Then

$$
\widetilde{\psi}: \Gamma \times(-1,1) \rightarrow \Gamma \times(-1,1), \quad(y, t) \mapsto(y, t-\sigma(t) \widetilde{\beta}(y))
$$

is an orientation preserving $C^{2}$ diffeomorphism onto $\Gamma \times(-1,1)$. It satisfies

$$
\widetilde{\psi}(y, t)=(y, t) \text { for } y \in \Gamma \text { and }|t| \geq 2 \widetilde{\delta} \text { and } \widetilde{\psi}(\widetilde{\Gamma})=\Gamma \times\{0\} .
$$

Let $\widetilde{\varphi}$ be the restriction of $\widetilde{\psi}^{-1}$ to

$$
\{(y, t) \in \Gamma \times(-1,1) ; t \geq 0\}
$$


and set

$$
\widehat{\varphi}:=h^{-1} \circ \tilde{\varphi} \circ h .
$$

Then $\widehat{\varphi}$ is a $C^{2}$ diffeomorphism from $\bar{\Omega} \cap T_{\rho}$ onto $\bar{\Omega}_{\beta} \cap T_{\rho}$ satisfying $\widehat{\varphi}(x)=x$ for $x \in \bar{\Omega} \cap T_{\rho}$ with dist $(x, \Gamma) \geq 2\|\beta\|_{\infty}$. Define $\varphi: \bar{\Omega} \rightarrow \bar{\Omega}_{\beta}$ by setting $\varphi(x):=\widehat{\varphi}(x)$ for $x \in \bar{\Omega} \cap T_{\rho}$, and $\varphi(x):=x$ for $x \in \Omega \backslash T_{\rho}$. Then $\varphi$ is an orientation preserving $C^{2}$ diffeomorphism possessing the stated properties.

Now we can prove a more precise version of Theorem 22 in which $W_{2}^{1}$ convergence of the eigenfunction is replaced by $W_{q}^{1}$ convergence.

Theorem 49. Let $\Omega$ be bounded and suppose that conditions (33)-(39) be satisfied, except that $r, s$, and $t$ are replaced by $\xi, \eta$, and $\zeta$, respectively. Then the assertion of Theorem 22 are valid, provided the exponents 2 in (40) are replaced by $q$.

Proof. We can assume that $\left\|\beta_{i}\right\|_{\infty} \leq \rho / 3$ for all $i \in \mathbb{N}$. For each $i$ fix an orientation preserving $C^{2}$ diffeomorphism $\varphi_{1}: \bar{\Omega} \rightarrow \bar{\Omega}_{\beta_{i}}$ such that $\varphi_{i}(x)=x$ for $x \in \Omega$ with $\operatorname{dist}(x, \Gamma) \geq 2\left\|\beta_{i}\right\|_{\infty}$. Lemma 48 guarantees that this is possible, and it follows from (94) and $\beta_{i} \rightarrow 0$ in $C^{1}(\Gamma, \mathbb{R})$ that $\varphi_{i} \rightarrow \mathrm{id}_{\bar{\Omega}}$ in $C^{1}\left(\bar{\Omega}, \mathbb{R}^{n}\right)$ as $i \rightarrow \infty$. It is easily verified that $\varphi_{i}^{*} \alpha_{i} \rightarrow \alpha$ in $\mathbb{E}_{\xi, \eta, \zeta}(\Omega)$. Hence Theorem 47 implies the assertion.

Similarly as above, it is clear that Theorem 49 contains Theorem 22 as a particular case.

\section{Elliptic comparison theorems}

In this section we study the weak maximum principle under optimal regularity assumptions for the coefficients of $\mathcal{A}$ and $\mathcal{B}$. More precisely, we assume that

- $\quad \xi, \eta$, and $\zeta$ satisfy (66), (67), and (68), respectively;

- $a_{j k}=a_{k j} \in B U C\left(\Omega, \mathbb{R}_{\text {diag }}^{N \times N}\right), 1 \leq j, k \leq n$;

- $\boldsymbol{a}^{r}(x) \in \mathbb{R}^{n \times n}$ is positive definite for $1 \leq r \leq N$, uniformly with respect to $x \in \Omega$;

- $\quad a_{j} \in\left(L_{\xi}+L_{\infty}\right)\left(\Omega, \mathbb{R}_{\text {diag }}^{N \times N}\right), 1 \leq j \leq n$;

- $\quad a \in\left(L_{\eta}+L_{\infty}\right)\left(\Omega, \mathbb{R}^{N \times N}\right), b \in L_{\zeta}\left(\Gamma, \mathbb{R}^{N \times N}\right)$;

- $\quad-a$ and $-b$ are cooperative;

- $\chi$ is a boundary characterization map for $\Omega$.

Then we define $(\mathcal{A}, \mathcal{B})$ and its Dirichlet form as before. Note that

$$
W_{q, \mathcal{B}}^{1}:=W_{q,(1-\chi) \gamma}^{1} \quad \text { and } \quad W_{q, \mathcal{B}}^{-1}:=\left(W_{q, \mathcal{B}}^{1}\right)^{\prime}
$$

are still well-defined. 
It follows from Lemma 45 that

$$
\mathfrak{a} \in \mathcal{L}\left(W_{q^{\prime},(1-\chi) \gamma}^{1}, W_{q,(1-\chi) \gamma}^{1} ; \mathbb{R}\right) .
$$

Hence there exits a unique

$$
A_{-1} \in \mathcal{L}\left(W_{q, \mathcal{B}}^{1}, W_{q, \mathcal{B}}^{-1}\right)
$$

satisfying

$$
\left\langle v, A_{-1} u\right\rangle=\mathfrak{a}(v, u), \quad(v, u) \in W_{q^{\prime},(1-\chi) \gamma}^{1} \times W_{q,(1-\chi) \gamma}^{1},
$$

the $W_{q, \mathcal{B}}^{-1}$ realization of $(\mathcal{A}, \mathcal{B})$.

Observe that in the present situation $\mathcal{A}\left(W_{q}^{2}\right) \not \subset L_{q}$, in general, so that the $L_{q}$ realization of $(\mathcal{A}, \mathcal{B})$ cannot be defined as in the earlier sections and is not useful for our purposes.

Theorem 50. Let (95) be satisfied. Then

$$
A_{-1} \in \mathcal{H}\left(W_{q, \mathcal{B}}^{1}, W_{q, \mathcal{B}}^{-1}\right)
$$

and is resolvent positive.

Proof. In [9] it is shown (by an amplification of the proof of [4, Theorem 2.1], where $\Omega$ is supposed to be bounded and more regularity is required for the lower order coefficients) that $A_{-1} \in \mathcal{H}\left(W_{q, \mathcal{B}}^{1}, W_{q, \mathcal{B}}^{-1}\right)$.

Choose a sequence $\alpha_{k} \in \mathbb{E}^{1}(\Omega)$ converging in $\mathbb{E}_{\xi, \eta, \zeta}(\Omega)$ towards

$$
\alpha:=\left(\left[a_{j k}\right],\left(a_{1}, \ldots, a_{n}\right), a, b, \chi\right) .
$$

Since $\mathcal{D}$ is dense in $L_{p}$ for $1 \leq p<\infty$, it is clear that such a sequence exists. Then Lemma 45 and (69) imply that

$$
A_{-1}\left(\alpha_{k}\right) \rightarrow A_{-1} \quad \text { in } \mathcal{L}\left(W_{q, \mathcal{B}}^{1}, W_{q, \mathcal{B}}^{-1}\right) .
$$

By Theorems 37 and 41 we know that

$$
A_{-1}\left(\alpha_{k}\right) \in \mathcal{H}\left(W_{q, \mathcal{B}}^{1}, W_{q, \mathcal{B}}^{-1}\right)
$$

and is resolvent positive. Thus Proposition 34 implies that $A_{-1}$ is also resolvent positive.

Clearly, definition (18) of the weak maximum principle is valid in the present situation also, as is the definition (21) of a (weak) $W_{q}^{1}$ solution.

Corollary 51. Let assumption (95) be satisfied and suppose that

$$
\lambda_{0}\left(A_{-1}\right)=-s\left(-A_{-1}\right)>0
$$

Then

(i) $(\mathcal{A}, \mathcal{B})$ satisfies the weak maximum principle in $W_{q}^{1}$; 
(ii) The boundary value problem

$$
\begin{gathered}
\mathcal{A} u=f \text { in } \Omega, \quad \mathcal{B} u=g \text { on } \Gamma \\
\text { has for each }(f, g) \in W_{q, \mathcal{B}}^{-1} \times \partial W_{q}^{1} \text { a unique } W_{q}^{1} \text { solution } u \text {, and } \\
u \geq 0 \quad \text { if }(f, g) \geq 0 .
\end{gathered}
$$

Proof. (i) follows from Theorems 28 and 50.

(ii) Assumption (96) guarantees that $0 \in \rho\left(A_{-1}\right)$. Thus the proof of case $j=1$ of Theorem 39(ii) applies to give the unique solvability. The last part of the assertion is a consequence of (i).

\section{Remarks 52.}

(a) Note that Theorem 28(b) guarantees that $\lambda_{0}\left(-A_{-1}\right)$ belongs to $\sigma\left(A_{-1}\right)$ if the latter set is not empty. However, we do not know whether this is true, in general, even if the domain is bounded (in which case $A_{-1}$ has a compact resolvent) and even if $N=1$.

(b) In the weak setting studied above it is natural to consider operators of the form

$$
\widetilde{\mathcal{A}} u:=-\partial_{j}\left(a_{j k} \partial_{k} u+\widetilde{a}_{j} u\right)+a_{j} \partial_{j}+a u,
$$

where $\widetilde{a}_{j}: \Omega \rightarrow \mathbb{R}_{\mathrm{diag}}^{N \times N}$ satisfy appropriate regularity assumptions. It is not difficult to determine these optimal conditions and to show that Theorem 50 and its corollary hold in this case also. Note that the corresponding boundary operator is now formally — given by

$$
\widetilde{\mathcal{B}} u:=\chi\left(\partial_{\mu} u+\nu^{j} \cdot \widetilde{a}_{j} u+b u\right)+(1-\delta) u .
$$

We leave the details to the interested reader.

The scalar case $(N=1$ has been studied by many authors (see [23], [24], [38], [49], [61], [68], [73]-[75], and the references therein). However, in all those papers only the case $q=2$ is considered. In that situation one can, of course, weaken the regularity conditions on $\Gamma$ considerably, and it suffices to assume that the $a_{j k}$ are only bounded and measurable (in fact, Trudinger [73]-[75] considers even the case of nonuniformly elliptic equations). It is well-known that this is no longer true if $q \neq 2$. We do not know of any work dealing with weak maximum principles in a $W_{q}^{1}$ setting, except for [7, Theorem 8.7], where the resolvent positivity of $A_{-1}$ is proved if $N=1$ and the lower order coefficients satisfy stronger regularity assumptions.

Now we can easily derive a comparison theorem for semilinear elliptic boundary value problems. For this we recall that, given a $\sigma$-finite measure space $(X, m)$ and Banach spaces $E$ and $F$, a function $f: X \times E \rightarrow F$ is said to be a Carathéodory function if

$$
f(x, \cdot): E \rightarrow F
$$

is continuous for $m$-a.a. $x \in X$, and

$$
f(\cdot, \xi): X \rightarrow F
$$


is $m$-measurable for each $\xi \in E$. We denote by $\operatorname{Car}(X \times E, F)$ the set of all such functions.

We assume that

- $f \in \operatorname{Car}\left(\Omega \times \mathbb{R}^{N}, \mathbb{R}^{N}\right), g_{1} \in \operatorname{Car}\left(\Gamma \times \mathbb{R}^{N}, \mathbb{R}^{N}\right)$;

- $r, s \in(1, \infty)$ with $r \leq(n+q) /(n-q)$ and $s \leq n /(n-q)$ if $q<n$;

- $\alpha_{0} \in L_{r_{0}}\left(\Omega, \mathbb{R}^{+}\right)$and $\beta_{0} \in L_{s_{0}}\left(\Gamma, \mathbb{R}^{+}\right)$, where $r_{0}, s_{0} \in[1, \infty)$ satisfy $r_{0} \geq n q /(n+q)$ and $s_{0} \geq q(n-1) / n$;

- $|f(\cdot, \xi)| \leq \alpha_{0}+\alpha|\xi|^{r},\left|g_{1}(\cdot, \xi)\right| \leq \beta_{0}+\beta|\xi|^{s}, \xi \in \mathbb{R}^{N}$, where $\alpha$ and $\beta$ are positive constants;

- $g_{0} \in W_{q}^{1-1 / q}\left(\Gamma, \mathbb{R}^{N}\right)$.

We also set

$$
g(\cdot, \xi):=(1-\chi) g_{0}+\chi g_{1}(\cdot, \xi), \quad \xi \in \mathbb{R}^{N},
$$

as well as

$$
F(u):=f(\cdot, u(\cdot)), \quad G(u):=g(\cdot, \gamma u(\cdot)) .
$$

Then Sobolev embeddings, the trace theorem, and an obvious duality argument imply that

$$
(F(u), G(u)) \in W_{q, \mathcal{B}}^{-1} \times \partial W_{q}^{1}, \quad u \in W_{q}^{1} .
$$

A function $\widehat{u}$ is said to be a $W_{q}^{1}$ supersolution of the nonlinear boundary value problem

$$
\mathcal{A} u=f(x, u) \text { in } \Omega, \quad \mathcal{B} u=g(x, u) \text { on } \Gamma
$$

if $\widehat{u} \in W_{q}^{1}$ and

$$
\mathcal{A} \widehat{u} \geq f(x, \widehat{u}) \text { in } \Omega, \quad \mathcal{B} \widehat{u} \geq g(x, \widehat{u}) \text { on } \Gamma
$$

in the weak sense, that is,

$$
\left.\begin{array}{rlrl}
\mathfrak{a}(v, \widehat{u}) & \geq\langle v, F(\widehat{u})\rangle+\langle\gamma v, G(\widehat{u})\rangle_{\Gamma} & & \text { for } v \in\left(W_{q^{\prime},(1-\chi) \gamma}^{1}\right)^{+}, \\
(1-\chi) \gamma \widehat{u} & \geq(1-\chi) g_{0} & & \text { on } \Gamma .
\end{array}\right\}
$$

If both inequalities in (99) are reversed then $\widehat{u}$ is said to be a $W_{q}^{1}$ subsolution of (98).

Theorem 53. Let (95) and (97) be satisfied and suppose that

$$
\lambda_{0}\left(A_{-1}\right)>0 .
$$

If $\bar{u}$ is a $W_{q}^{1}$ subsolution and $\widehat{u}$ is $a W_{q}^{1}$ supersolution of (98) such that

$$
(F(\bar{u}), G(\bar{u})) \leq(F(\widehat{u}), G(\widehat{u}))
$$

then

$$
\bar{u} \leq \widehat{u}
$$


Proof. The assumptions imply that $\widehat{u}-\bar{u} \in W_{q}^{1}$ satisfies

$$
\begin{aligned}
\mathfrak{a}(v, \widehat{u}-\bar{u}) \geq 0 & & \text { for } v \in\left(W_{q^{\prime},(1-\chi) \gamma}^{1}\right)^{+}, \\
(1-\chi) \gamma(\widehat{u}-\bar{u}) \geq 0 & & \text { on } \Gamma .
\end{aligned}
$$

Hence the assertion follows from Corollary 51.

It should be clear to the reader that in order to guarantee the validity of the very weak maximum principle the regularity assumption on $a$ in (7) can be weakened also. We refrain from giving details.

\section{References}

1. Amann, H., Fixed point equations and nonlinear eigenvalue problems in ordered Banach spaces, SIAM Rev. 18 (1976), 620-709.

2. Amann, H., Nonlinear elliptic equations with nonlinear boundary conditions, in New Developments in Differential Equations (Proc. 2nd Scheveningen Conf., Scheveningen, 1975), pages 43-63, North-Holland Math. Studies 21, North-Holland, Amsterdam, 1976.

3. Amann, H., Dual semigroups and second order linear elliptic boundary value problems, Israel J. Math. 45 (1983), 225-254.

4. Amann, H., Dynamic theory of quasilinear parabolic systems, III. Global existence, Math. Z. 202 (1989), 219-250.

5. Amann, H., Dynamic theory of quasilinear parabolic equations, II. Reaction-diffusion systems, Diff. Int. Eqns. 3 (1990), 13-75.

6. Amann, H., Ordinary Differential Equations, de Gruyter Studies in Mathematics 13, Walter de Gruyter \& Co., Berlin, 1990.

7. Amann, H., Nonhomogeneous linear and quasilinear elliptic and parabolic boundary value problems, in Function Spaces, Differential Operators and Nonlinear Analysis (Friedrichroda, 1992), pages 9-126, Teubner-Texte Math. 133, Teubner, Stuttgart, 1993.

8. Amann, H., Linear and Quasilinear Parabolic Problems, Vol. I, Monographs in Mathematics 89. Birkhäuser Boston Inc., Boston, MA, 1995.

9. Amann, H., Maximal regularity and weak solutions of linear parabolic equations, to appear.

10. Amann, H., and Escher, J., Analysis, Vol. III, Grundstudium Mathematik, Birkhäuser Verlag, Basel, 2001.

11. Amann, H., and López-Gómez, J., A priori bounds and multiple solutions for superlinear indefinite elliptic problems, J. Diff. Eqns. 146 (1998), 336-374.

12. Amann, H., and Quittner, P., Elliptic boundary value problems involving measures: existence, regularity, and multiplicity, Adv. Diff. Eqns. 3 (1998), 753-813.

13. Arendt, W., Gaussian estimates and interpolation of the spectrum in $L^{p}$, Diff. Int. Eqns. 7 (1994), 1153-1168. 
14. Arendt, W., and Batty, Ch.J.K., Principal eigenvalues and perturbation, in Operator Theory in Function Spaces and Banach Lattices, pages 39-55, Oper. Theory Adv. Appl. 75, Birkhäuser, Basel, 1995.

15. Arendt, W., Batty, Ch.J.K., Hieber, M., and Neubrander, F., Vector-valued Laplace Transforms and Cauchy Problems, Monographs in Mathematics 96, Birkhäuser Verlag, Basel, 2001.

16. Arendt, W., Grabosch, A., Greiner, G., Groh, U., Lotz, H.P., Moustakas, U., Nagel, R., Neubrander, F., and Schlotterbeck, F., One-parameter Semigroups of Positive Operators, Lecture Notes in Mathematics 1184, Springer-Verlag, Berlin, 1986.

17. Arendt, W., and Monniaux, S., Domain perturbation for the first eigenvalue of the Dirichlet Schrödinger operator, in Partial Differential Operators and Mathematical Physics (Holzhau, 1994), pages 1-19, Oper. Theory Adv. Appl. 78, Birkhäuser, Basel, 1995.

18. Beltramo, A., and Hess, P., On the principal eigenvalue of a periodic-parabolic operator, Comm. Part. Diff. Eqns. 9 (1984), 919-941.

19. Berestycki, H., Nirenberg, L., and Varadhan, S.R.S., The principal eigenvalue and maximum principle for second-order elliptic operators in general domains, Comm. Pure Appl. Math. 47 (1994), 47-92.

20. Birindelli, I., Mitidieri, E., and Sweers, G., Existence of the principal eigenvalue for cooperative elliptic systems in a general domain, Differ. Uravn. 35 (1999), 325-333.

21. Cano-Casanova, S., and López-Gómez, J., Properties of the principal eigenvalues of a general class of non-classical mixed boundary value problems, J. Diff. Eqns. 178 (2002), 123-211.

22. Cantrell, R.S., and Schmitt, K., On the eigenvalue problem for coupled elliptic systems, SIAM J. Math. Anal. 17 (1986), 850-862.

23. Chicco, M., Principio di massimo generalizzato e valutazione del primo autovalore per problemi ellittici del secondo ordine di tipo variazionale, Ann. Mat. Pura Appl. (4) 87 (1970), 1-9.

24. Chicco, M., Some properties of the first eigenvalue and the first eigenfunction of linear second order elliptic partial differential equations in divergence form, Boll. Un. Mat. Ital. 5 (1972), 245-254.

25. Clément, Ph., Heijmans, H.J.A.M., Angenent, S., van Duijn, C.J., and de Pagter, B., One-Parameter Semigroups, CWI Monographs 5, North-Holland Publishing Co., Amsterdam, 1987.

26. Clément, Ph., and Peletier, L.A., An anti-maximum principle for second-order elliptic operators, J. Diff. Eqns. 34 (1979), 218-229.

27. Dancer, E.N., and Daners, D., Domain perturbation for elliptic equations subject to Robin boundary conditions, J. Diff. Eqns. 138 (1997), 86-132.

28. Daners, D., Existence and perturbation of principal eigenvalues for a periodic-parabolic problem, in Proceedings of the Conference on Nonlinear Differential Equations (Coral Gables, FL, 1999), pages 51-67 (electronic), Electron. J. Differ. Equ. Conf. 5, San Marcos, TX, 2000.

29. Daners, D., Dirichlet problems on varying domains, J. Diff. Eqns. 188 (2003), 591-624.

30. Dautray, R., and Lions, J.L., Mathematical Analysis and Numerical Methods for Science and Technology, Vol. 1, Springer-Verlag, Berlin, 1990. 
31. Davies, E.B., $L^{p}$ spectral independence for certain uniformly elliptic operators, in Partial differential Equations and Mathematical Physics (Copenhagen, 1995; Lund, 1995), pages 122-125, Progr. Nonlinear Differential Equations Appl. 21, Birkhäuser Boston, Boston, MA, 1996.

32. de Figueiredo, D.G., and Mitidieri, E., Maximum principles for cooperative elliptic systems, C. R. Acad. Sci. Paris Sér. I Math. 310 (1990), 49-52.

33. de Pagter, B., Irreducible compact operators, Math. Z. 192 (1986), 149-153.

34. Denk, R., Hieber, M., and Prüss, J., $\mathcal{R}$-boundedness, Fourier Multipliers and Problems of Elliptic and Parabolic Type, Memoirs Amer. Math. Soc. 166, Providence, R.I., 2003.

35. Fleckinger, J., Hernández, J., and de Thélin, F., Existence of multiple principal eigenvalues for some indefinite eigenvalue problems, Boll. Unione Mat. Ital. 7 (2004), 159-188.

36. Fleckinger, J., Hernández, J., and de Thélin, F., A maximum principle for linear cooperative elliptic systems, in Differential Equations with Applications to Mathematical Physics, pages 79-86, Math. Sci. Engrg. 192, Academic Press, Boston, MA, 1993.

37. Fraile, J.M., Koch Medina, P., López-Gómez, J., and Merino, S., Elliptic eigenvalue problems and unbounded continua of positive solutions of a semilinear elliptic equation, J. Diff. Eqns. 127 (1996), 295-319.

38. Gilbarg, D., and Trudinger, N.S., Elliptic Partial Differential Equations of Second Order, Classics in Mathematics, Springer-Verlag, Berlin, 2001.

39. Greiner, G., Zur Perron-Frobenius-Theorie stark stetiger Halbgruppen, Math. Z. 177 (1981), 401-423.

40. Hernández, J., Some existence and stability results for solutions of reaction-diffusion systems with nonlinear boundary conditions, in Nonlinear Differential Equations (Proc. Internat. Conf., Trento, 1980), pages 161-173, Academic Press, New York, 1981.

41. Hernández, J., Positive solutions of reaction-diffusion systems with nonlinear boundary conditions and the fixed point index, in Nonlinear Phenomena in Mathematical Sciences (Arlington, Tex., 1980), pages 525-535, Academic Press, New York, 1982.

42. Hernández, J., Mancebo, F.J., and Vega, J.M., On the linearization of some singular, nonlinear elliptic problems and applications, Analyse Non Linéaire, Ann. Inst. H. Poincaré 19 (2002), 777-813.

43. Hess, P., On the eigenvalue problem for weakly coupled elliptic systems, Arch. Rat. Mech. Anal. 81 (1983), 151-159.

44. Hess, P., Periodic-Parabolic Boundary Value Problems and Positivity, Pitman Research Notes in Mathematics Series 247, Longman Scientific \& Technical, Harlow, 1991.

45. Hieber, M., and Schrohe, E., $L^{p}$ spectral independence of elliptic operators via commutator estimates, Positivity 3 (1999), 259-272.

46. Hirsch, M.W., Differential Topology, Graduate Texts in Mathematics 33, SpringerVerlag, New York, 1976.

47. Kato, T., Superconvexity of the spectral radius, and convexity of the spectral bound and the type, Math. Z. 180 (1982), 265-273.

48. Kato, T., Perturbation Theory for Linear Operators, Classics in Mathematics, SpringerVerlag, Berlin, 1995. 
49. Kinderlehrer, D., and Stampacchia, G., An Introduction to Variational Inequalities and their Applications, Classics in Applied Mathematics 31, Society for Industrial and Applied Mathematics (SIAM), Philadelphia, PA, 2000.

50. Kunstmann, P.Ch., Heat kernel estimates and $L^{p}$ spectral independence of elliptic operators, Bull. London Math. Soc. 31 (1999), 345-353.

51. López-Gómez, J., Nonlinear eigenvalues and global bifurcation application to the search of positive solutions for general Lotka-Volterra reaction diffusion systems with two species, Diff. Int. Eqns. 7 (1994), 1427-1452.

52. López-Gómez, J., The maximum principle and the existence of principal eigenvalues for some linear weighted boundary value problems, J. Diff. Eqns. 127 (1996), 263-294.

53. López-Gómez, J., Large solutions, metasolutions, and asymptotic behaviour of the regular positive solutions of sublinear parabolic problems, in Proceedings of the Conference on Nonlinear Differential Equations (Coral Gables, FL, 1999), pages 135171 (electronic), Electron. J. Differ. Equ. Conf. 5, San Marcos, TX, 2000.

54. López-Gómez, J., Spectral Theory and Nonlinear Functional Analysis, Chapman \& Hall/CRC Research Notes in Mathematics 426, Chapman \& Hall/CRC, Boca Raton, FL, 2001.

55. López-Gómez, J., Classifying smooth supersolutions in a general class of elliptic boundary value problems, Adv. in Diff. Eqns. 8 (2003), 1025-1042.

56. López-Gómez, J., and Molina-Meyer, M., The maximum principle for cooperative weakly coupled elliptic systems and some applications, Diff. Int. Eqns. 7 (1994), 383398.

57. López-Gómez, J., and Pardo, R., Multiparameter nonlinear eigenvalue problems: positive solutions to elliptic Lotka-Volterra systems, Appl. Anal. 31 (1988), 103-127.

58. López-Gómez, J., and Sabina de Lis, J., Coexistence states and global attractivity for some convective diffusive competing species models, Trans. Amer. Math. Soc. 347 (1995), 3797-3833.

59. Mitidieri, E., and Sweers, G., Existence of a maximal solution for quasimonotone elliptic systems, Diff. Int. Eqns. 7 (1994), 1495-1510.

60. Mitidieri, E., and Sweers, G., Weakly coupled elliptic systems and positivity, Math. Nachr. 173 (1995), 259-286.

61. Nečas, J., Les Méthodes Directes en Théorie des Équations Elliptiques, Masson et Cie, Éditeurs, Paris, 1967.

62. Pao, C.V., Nonlinear Parabolic and Elliptic Equations, Plenum Press, New York, 1992.

63. Protter, M.H., and Weinberger, H.F., Maximum Principles in Differential Equations, Springer-Verlag, New York, 1984.

64. Sattinger, D.H., Monotone methods in nonlinear elliptic and parabolic boundary value problems, Indiana Univ. Math. J. 21 (1971), 979-1000.

65. Schaefer, H.H., Topological Vector Spaces, Graduate Texts in Mathematics 3, SpringerVerlag, New York, 1971.

66. Schmitt, K., Boundary value problems for quasilinear second-order elliptic equations, Nonlinear Anal. 2 (1978), 263-309.

67. Smoller, J., Shock Waves and Reaction-Diffusion Equations, Grundlehren der Mathematischen Wissenschaften 258, Springer-Verlag, New York, 1983. 
68. Stampacchia, G., Le problème de Dirichlet pour les équations elliptiques du second ordre à coefficients discontinus, Ann. Inst. Fourier (Grenoble) 15 (1965), 189-258.

69. Stollmann, P., A convergence theorem for Dirichlet forms with applications to boundary value problems with varying domains, Math. Z. 219 (1995), 275-287.

70. Sturm, K.Th., On the $L^{p}$-spectrum of uniformly elliptic operators on Riemannian manifolds, J. Funct. Anal. 118 (1993), 442-453.

71. Sweers, G., Strong positivity in $C(\bar{\Omega})$ for elliptic systems. Math. Z. 209 (1992), 251271.

72. Takáč, P., An abstract form of maximum and anti-maximum principles of Hopf's type, J. Math. Anal. Appl. 201 (1996), 339-364.

73. Trudinger, N.S., Maximum principles for linear, non-uniformly elliptic operators with measurable coefficients, Math. Z. 156 (1977), 291-301.

74. Trudinger, N.S., On the positivity of weak supersolutions of nonuniformly elliptic equations, Bull. Austral. Math. Soc. 19 (1978), 321-324.

75. Trudinger, N.S., On the first eigenvalue of nonuniformly elliptic boundary value problems, Math. Z. 174 (1980), 227-232.

76. Walter, W., A theorem on elliptic differential inequalities with an application to gradient bounds, Math. Z. 200 (1989), 293-299.

77. Walter, W., The minimum principle for elliptic systems, Appl. Anal. 47 (1992), 1-6. 